\title{
Observational constraints on the origin of the elements
}

\section{3D NLTE formation of Mn lines in late-type stars $\star, \star \star$}

\author{
Maria Bergemann ${ }^{1}$, Andrew J. Gallagher ${ }^{1}$, Philipp Eitner ${ }^{1,2}$, Manuel Bautista ${ }^{3}$, Remo Collet $^{4}$, Svetlana A. Yakovleva ${ }^{5}$,
} Anja Mayriedl ${ }^{6}$, Bertrand Plez ${ }^{7}$, Mats Carlsson ${ }^{8,9}$, Jorrit Leenaarts ${ }^{10}$, Andrey K. Belyaev ${ }^{5}$, and Camilla Hansen ${ }^{1}$

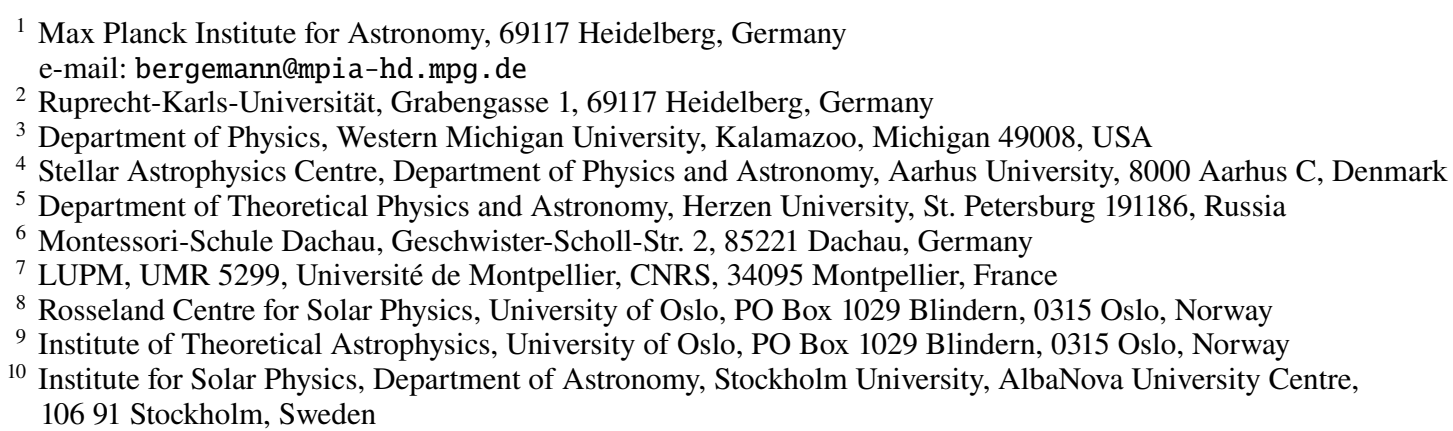

Received 30 April 2019 / Accepted 12 June 2019

\begin{abstract}
Manganese (Mn) is a key Fe-group element, commonly employed in stellar population and nucleosynthesis studies to explore the role of SN Ia. We have developed a new non-local thermodynamic equilibrium (NLTE) model of Mn, including new photo-ionisation cross-sections and new transition rates caused by collisions with $\mathrm{H}$ and $\mathrm{H}^{-}$atoms. We applied the model in combination with onedimensional (1D) LTE model atmospheres and 3D hydrodynamical simulations of stellar convection to quantify the impact of NLTE and convection on the line formation. We show that the effects of NLTE are present in Mn I and, to a lesser degree, in Mn II lines, and these increase with metallicity and with the effective temperature of a model. Employing 3D NLTE radiative transfer, we derive a new abundance of $\mathrm{Mn}$ in the Sun, $A(\mathrm{Mn})=5.52 \pm 0.03 \mathrm{dex}$, consistent with the element abundance in C I meteorites. We also applied our methods to the analysis of three metal-poor benchmark stars. We find that 3D NLTE abundances are significantly higher than 1D LTE. For dwarfs, the differences between 1D NLTE and 3D NLTE abundances are typically within 0.15 dex, however, the effects are much larger in the atmospheres of giants owing to their more vigorous convection. We show that 3D NLTE successfully solves the ionisation and excitation balance for the RGB star HD 122563 that cannot be achieved by 1D LTE or 1D NLTE modelling. For HD 84937 and HD 140283, the ionisation balance is satisfied, however, the resonance Mn I triplet lines still show somewhat lower abundances compared to the high-excitation lines. Our results for the benchmark stars confirm that 1D LTE modelling leads to significant systematic biases in Mn abundances across the full wavelength range from the blue to the IR. We also produce a list of Mn lines that are not significantly biased by 3D and can be reliably, within the 0.1 dex uncertainty, modelled in 1D NLTE.
\end{abstract}

Key words. stars: abundances - Sun: abundances - stars: atmospheres - Sun: atmosphere - line: formation - radiative transfer

\section{Introduction}

Manganese $(\mathrm{Mn})$ is a prominent member of the iron-group family that has interesting connections to several topics in astrophysics. In particular, from the point of view of stellar nucleosynthesis, this element is very sensitive to the physical conditions in supernovae Type Ia (SNIa; Seitenzahl et al. 2013). Hence, the abundances of $\mathrm{Mn}$ in metal-poor stars provide powerful constraints on the progenitors and explosion mechanism of this important class of $\mathrm{SNe}$.

Mn displays a large number of Mn I lines spanning a range of excitation potentials in the optical spectra of late-type stars

\footnotetext{
* The new cross-sections and rate coefficients are only available at the CDS via anonymous ftp to cdsarc.u-strasbg.fr (130.79.128.5) or via http://cdsarc.u-strasbg.fr/viz-bin/cat/J/A+A/631/ A80

$\star \star$ The atomic model is available at https://keeper.mpdl.
} mpg.de/f/1ce2a838074b49fc9424/?dl=1
(Bergemann \& Gehren 2007). Also a few lines of Mn II can be detected in the blue at $\sim 350 \mathrm{~nm}$, and some strong lines of Mn I are available in the IR at $1.52 \mu \mathrm{m}$. Owing to the large number of observable lines, $\mathrm{Mn}$ is a useful element to test the excitation and ionisation equilibria in stellar atmospheres. The lines of both ionisation stages are affected by hyperfine splitting (HFS), and some are also very sensitive to stellar activity. For example, the resonance Mn I line at $5394 \AA$ is known to vary across the solar cycle (Vitas et al. 2009; Danilovic et al. 2016).

A large number of studies over the past years have been devoted to the analysis of Mn abundances in the context of stellar population studies and nucleosynthesis. Most of these works have assumed local thermodynamic equilibrium (LTE). There is, however, evidence for the breakdown of the LTE assumption. Johnson (2002) reported a systematic ionisation imbalance of Mn I and Mn II in metal-poor stars. Bonifacio et al. (2009) found a 0.2 dex offset between the abundances of $\mathrm{Mn}$ in metalpoor dwarfs and giants. They also observe a significant excitation 
imbalance, with strong Mn I resonance lines resulting in significantly lower abundances compared to the high-excitation features. Sneden et al. (2016) confirm the excitation imbalance in LTE, but they also find that the ionisation balance is satisfied, if one relies on the high-excitation Mn I lines only. However, that study employed one star only, HD 84937, which can make it difficult to generalise these conclusions to a large sample. Mishenina et al. (2015) also employed LTE models to analyse a large sample of main-sequence stars in the metallicity range from -1 to +0.3 . Their abundances suggest a modest systematic correlation with $T_{\text {eff }}$, signifying potential departures from LTE and 1D hydrostatic equilibrium.

In earlier studies (Bergemann \& Gehren 2007, 2008), we showed that $\mathrm{Mn}$ is very sensitive to departures from LTE, also known as non-LTE (NLTE) effects. This is an element of the $\mathrm{Fe}$-group, and is expected to be similar to $\mathrm{Fe}$ in terms of line formation properties. However, $\mathrm{Mn}$ is prone to stronger NLTE effects than Fe given its lower abundance of two orders of magnitude (in the cosmic abundance scale) compared to $\mathrm{Fe}$, but also significantly higher photo-ionisation cross-sections, and a peculiar atomic structure with a very large number of strong radiative transitions between energy levels with excitation potentials of 2 and $4 \mathrm{eV}$. In particular, it was shown, on the basis of detailed statistical equilibrium (SE) calculations, that NLTE Mn abundances are significantly higher compared to LTE. This effect increases with decreasing metallicity and $\log g$ of a star, but also occurs with increasing $T_{\text {eff }}$.

Line formation across the solar granulation has been extensively discussed in the literature, in particular in the series of seminal papers by Dravins et al. (1981); Dravins (1987); Dravins \& Nordlund (1990a,b); Nordlund \& Dravins (1990), but see also more recent studies of the solar center-to-limb variation (e.g. Lind et al. 2017) and solar abundances (e.g. Caffau et al. 2008, 2009, 2010, 2011; Asplund et al. 2009; Amarsi \& Asplund 2017; Amarsi et al. 2018a, 2019). Recently, this work has been extended towards 3D NLTE modelling of spectral line formation in other stars and applied to the lines of $\mathrm{H}, \mathrm{O}, \mathrm{Si}$, and $\mathrm{Al}$ (Amarsi et al. 2016, 2018b, 2019; Nordlander \& Lind 2017).

Given the interest in the impact of NLTE and 3D diagnostic on the abundance measurements, we present a re-analysis of Mn abundances in a small sample of well-studied FGK stars using an updated NLTE model atom, and 1D hydrostatic and 3D hydrodynamical model atmospheres. We use new atomic data, including transition probabilities, photo-ionisation cross-sections, and rate coefficients for the transitions caused by the inelastic collisions of $\mathrm{MnI}$ and $\mathrm{Mn}$ II ions with $\mathrm{H}$ atoms. We compare the results of two 1D statistical equilibrium codes, DETAIL and MULTI2.3 that are both widely used in the community for NLTE analyses of chemical abundances. We also performed full 3D NLTE radiative transfer calculations with the MULTI3D code (Leenaarts \& Carlsson 2009) to derive Mn abundance from the high-resolution spectra of the Sun and several metal-poor stars.

The paper is organised as follows: in Sect. 2, we describe the observed spectra. The LTE and NLTE calculations in 1D and $3 \mathrm{D}$, spectrum synthesis, and abundance analysis are documented in Sect. 3. We present a considerable amount of details about the methods of calculations, as this is very important for a judgement of the resulting abundances. The results are presented in Sect. 4. This section includes a discussion of 1D NLTE and 3D NLTE abundance corrections, an analysis of the solar Mn abundance, a comparison between 3D LTE and 3D NLTE line profiles, and the excitation-ionisation balance of Mn I/Mn II in benchmark metal-poor stars. We close with a summary of the results and conclusions in Sect. 5.

\section{Observations}

For the Sun, we used the high-resolution flux atlas taken with the KPNO facility (Kurucz et al. 1984). The atlas has a resolving power $R \sim 400000$. Recently, solar spectra taken with the PEPSI instrument at Large Binocular Telescope (LBT; Strassmeier et al. 2018) and with the Fourier transform spectrograph operated by the Institut fuer Astrophysik in Goettingen (Reiners et al. 2016) were released. However, the profiles of Mn lines are very similar in all these atlases. For this reason, we employed the KPNO spectrum in this work.

We also included several metal-poor benchmark stars (HD 84937, HD 140283, and HD 122563) from Bergemann et al. (2012). Their spectra are taken from the UVES-POP database (Bagnulo et al. 2003). These are the Gaia benchmark stars with $T_{\text {eff }}$ and $\log g$ determined using interferometry and astrometry. The estimates of $[\mathrm{Fe} / \mathrm{H}]$ and micro-turbulence were derived using NLTE radiative transfer for Fe lines (Bergemann et al. 2012). The effective temperatures of two of these stars were recently revised (Karovicova et al. 2018). The new estimates, based on the CHARA angular diameters, are $T_{\text {eff }}=5787 \pm 48 \mathrm{~K}$ for HD 140283 and $T_{\text {eff }}=4636 \pm 37 \mathrm{~K}$ for HD 122563. These estimates are fully consistent with the values we adopted in Bergemann et al. (2012). Creevey et al. (2019) propose a new asteroseismic surface gravity for HD 122563, $\log g=1.39 \pm$ 0.01 dex. This is a substantial downward revision of this parameter. However, we tested the effect of $\log g$ on the abundance estimates from Mn lines, but found that the abundances change by only 0.05 dex. Hence, we did not recompute the model and used instead the standard models employed in Bergemann et al. (2012).

\section{Analysis}

\subsection{Model atom and diagnostic lines of $\mathrm{Mn}$}

The model comprises three ionisation stages and 281 energy levels, with 198 levels of Mn I and 81 levels of Mn II. The model is also closed by the Mn III ground state. The radiative transitions were taken from the Kurucz compilation ${ }^{1}$, which includes theoretically predicted and experimental estimates of the oscillator strengths, with the latter given a preference over theoretical estimates. The Mn I part of the model atom is shown in Fig. 1. We do not show the Mn II system in this plot. The ionised species has a very high ionisation potential and the bulk of Mn II lines, which connect the levels do not play any role in the SE of the element (see also the discussion of model atom completeness in Bergemann \& Gehren 2008). In contrast to the latter study, we did not include fine structure for most of the Mn II levels, except those which are relevant for the Mn II near-UV lines used in detailed abundance measurements. The full atom is provided in the MULTI2.3 format at the CDS. We include fine structure levels for all energies up to $47300 \mathrm{~cm}^{-1}$, however, we also test the results using a compact model atom, which is devoid of fine structure for the majority of levels. This is important for our test calculations with full 3D simulations of stellar convection.

The atomic data for the Mn I lines, which we employ in the abundance calculations, are given in Table 1. For all of them, the HFS is included in the model atom directly. That is, the HFS structure of spectral lines was computed for all diagnostic lines of Mn I and Mn II, and included in the radiative transition part of the MULTI model atom. We employed the magnetic dipole constants $A$ and electric quadrupole constants $B$ assembled by

1 http://kurucz.harvard.edu/atoms/2500/ 


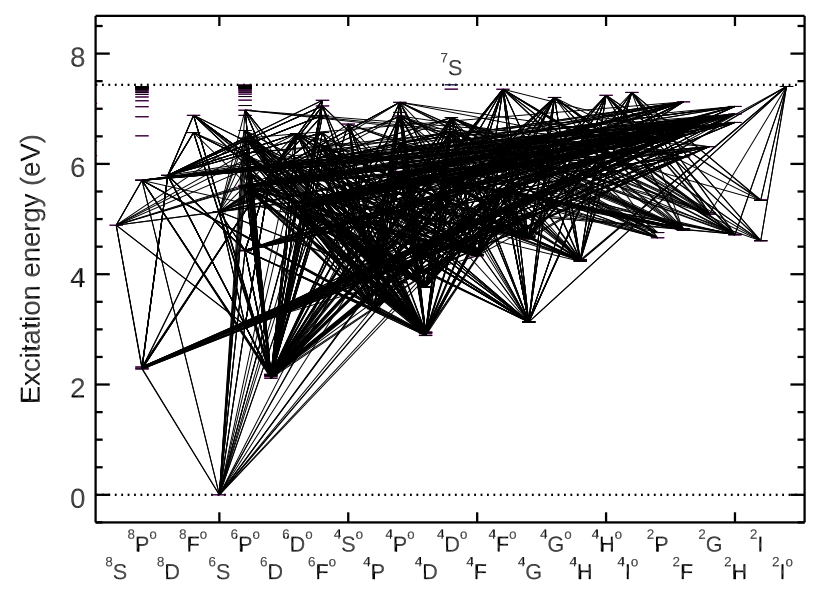

Fig. 1. Grotrian diagram of Mn I atomic system. The energy levels of Mn I are shown with black dashes. The levels are connected by radiative transitions (solid black lines).

Bergemann \& Gehren (2008), complementing these with the data from Holt et al. (1999) for the relevant Mn II levels, $a^{5} \mathrm{D}$ and $z^{5} \mathrm{P}^{\circ}$. The full HFS patterns for each Mn line is provided in supplementary material. In the SE calculations, we treated the diagnostic Mn I and Mn II lines with Voigt profiles, while all other Mn lines were computed with a Gaussian profile with 13 frequency points. Our tests show that increasing the number of frequency points does not change the statistical equilibrium of the ion, on the other hand with this choice we still have a reasonable frequency quadrature to accurately represent each line profile.

We used the new experimental transition probabilities, where these are available. Most data are from Blackwell-Whitehead \& Bergemann (2007) and Den Hartog et al. (2011). For some of the lines, the new $\log g f$ values are typically $0.05-0.1$ dex lower than the old values, that ultimately leads to slightly higher abundances compared to our earlier work.

The broadening due to elastic collisions with $\mathrm{H}$ atoms is adopted from Barklem et al. (2000), where available. These data were derived using the 2nd order Rayleigh-Schroedinger perturbation theory as formulated by Brueckner (1971) and later generalised to transitions between different azimuthal quantum number states by O'Mara (1976), Anstee \& O'Mara (1991, 1995). This theory offers a more accurate representation of the broadening caused by collisions with $\mathrm{H}$ atoms than the theory by Unsöld (1927, 1955). The latter theory assumes that only collisions at large separations between atoms can strongly influence the line broadening, thus severely underestimating the line strengths. The main difference in this work with respect to our earlier studies (Bergemann \& Gehren 2008) is the implementation of the new photo-ionisation cross-sections for Mn I and the new rates of inelastic collisions due to the interactions of Mn I with $\mathrm{HI}$ atoms.

\subsubsection{Photo-ionisation}

We adopted new quantum-mechanical photo-ionisation crosssections for $84 \mathrm{LS}$ terms of $\mathrm{MnI}$, which belong to the configurations $3 d^{6}, 3 d^{5} 4 s, 3 d^{5} 4 p$, and $3 d^{4} 4 s^{2}$. The photo-ionisation cross-sections for dipole that allowed transitions in Mn I were computed using the R-matrix method for atomic scattering calculations (Berrington et al. 1987). The solution of the Schrödinger equation for the $N+1$ electron system is found on
Table 1. Parameters of MnI and Mn II lines used for abundance calculations.

\begin{tabular}{|c|c|c|c|c|c|c|}
\hline $\begin{array}{c}\lambda \\
(\AA) \\
(\AA)\end{array}$ & $M$ & HFS & $\begin{array}{l}\text { low } \\
\text { eV) }\end{array}$ & & & $\log g f$ \\
\hline 88.68 & 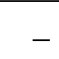 & 3 & 1.85 & & & 0.93 \\
\hline 96.81 & - & 2 & .83 & & & -1.779 \\
\hline 497.53 & - & 3 & 1.85 & $a^{5} \mathrm{D}_{1}^{2}$ & $z^{5} \mathrm{P}_{2}^{\mathrm{o}}$ & -1.418 \\
\hline 018.10 & 9 & 5 & 2.12 & $a^{6} \mathrm{D}_{9 / 2}$ & $z^{6} \mathrm{D}_{7 / 2}^{\mathrm{o}}$ & -0.311 \\
\hline 30.76 & 4 & 5 & 0.00 & & $z^{6} \mathrm{P}_{7 / 2}^{\mathrm{o}}$ & -0.497 \\
\hline 033.07 & 4 & 4 & .00 & 2 & $z^{6} \mathrm{P}_{5 / 2}^{\mathrm{o}}$ & .647 \\
\hline 34.49 & 4 & 3 & 0.00 & $a^{6} \mathrm{~S}_{5 / 2}$ & $z^{6} \mathrm{P}_{3 / 2}^{\mathrm{o}}$ & -0.843 \\
\hline 55. & 9 & 4 & 2.14 & $a^{6} \mathrm{D}_{7 / 2}$ & $z^{6} \mathrm{D}_{7 / 2}^{\mathrm{o}}$ & -0.077 \\
\hline 070.28 & 9 & 3 & 2.19 & $a^{6} \mathrm{D}_{1 / 2}$ & $z^{6} \mathrm{D}_{1 / 2}^{\mathrm{o}}$ & -1.039 \\
\hline 151.58 & 24 & 3 & 2.89 & $a^{4} \mathrm{D}_{7 / 2}^{1 / 2}$ & $z^{4} \mathrm{D}_{7 / 2}^{\mathrm{o}}$ & .278 \\
\hline 90 & 24 & 1 & 2. & $a^{4} \mathrm{D}_{2 / 2}$ & $z^{4} \mathrm{D}_{5 / 2}^{\mathrm{o}}$ & .343 \\
\hline 02.22 & 2 & 2 & 2.92 & $a^{4} \mathrm{D}_{5 / 2}^{2 / 2}$ & $z^{4} \mathrm{D}_{7 / 2}^{\mathrm{o}}$ & -0.345 \\
\hline 54.03 & 18 & 5 & 2.27 & $5 / 2$ & ${ }^{8} \mathrm{~S}_{7 / 2}$ & -0.086 \\
\hline 61.52 & 23 & 4 & 2.94 & $a^{4} \mathrm{D}_{1 / 2}^{5 / 2}$ & $z^{4} \mathrm{~F}_{3 / 2}^{\mathrm{o}}$ & -0.138 \\
\hline 762 & 23 & 5 & $2 . \varepsilon$ & $a^{4} \mathrm{D}_{7 / 2}$ & $z^{4} \mathrm{~F}_{9 / 2}^{\mathrm{o}}$ & .425 \\
\hline 65.86 & 2 & 3 & 2.93 & $a^{4} \mathrm{D}_{3 / 2}$ & $z^{4} \mathrm{~F}_{5 / 2}^{\mathrm{o}}$ & -0.080 \\
\hline 12 & 23 & 4 & 2.91 & $a^{4} \mathrm{D}^{3 / 2}$ & $z^{4} \mathrm{~F}_{7 / 2}^{\mathrm{o}}$ & 0.100 \\
\hline 12 & 18 & 5 & 2.2 & $z^{8} \mathrm{P}_{7 / 2}^{\mathrm{o}}$ & $e^{8} \mathrm{~S}_{7 / 2}$ & .042 \\
\hline 23. & 1 & 6 & & $z^{8} \mathrm{P}_{9 / 2}^{\mathrm{o}}$ & $e^{8} \mathrm{~S}_{7 / 2}$ & 0.144 \\
\hline 04.89 & 22 & 4 & 2.9 & $a^{4} \mathrm{D}_{5 / 2}$ & $z^{6} \mathrm{~F}_{7 / 2}^{\mathrm{o}}$ & -1.630 \\
\hline 17.93 & 39 & 3 & 3.12 & $a^{4} \mathrm{G}_{5 / 2}^{5 / 2}$ & $z^{4} \mathrm{~F}_{3 / 2}^{\mathrm{o}}$ & -1.140 \\
\hline 55.31 & 39 & 6 & 3.1 & $a^{4} \mathrm{G}_{11 / 2}$ & $z^{4} \mathrm{~F}_{9 / 2}^{\mathrm{o}}$ & -0.763 \\
\hline 394.67 & 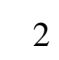 & 6 & 0.0 & $a^{6} \mathrm{~S}_{5 / 2}^{11 / 2}$ & $z^{8} \mathrm{P}_{7 / 2}^{\mathrm{o}}$ & -3.503 \\
\hline 407.42 & 8 & 10 & 2.13 & $6 \mathrm{D}^{3 / 2}$ & $y^{6} \mathrm{P}_{7 / 2}^{\mathrm{o}}$ & -1.743 \\
\hline 420.35 & 8 & ( & 2.1 & $a^{6} \mathrm{D}_{7 / 2}$ & ${ }^{6} \mathrm{P}_{5 / 2}^{\mathrm{o}}$ & -1.462 \\
\hline 32 & 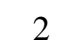 & s & 0.00 & & $z^{8} \mathrm{P}_{5 / 2}^{\mathrm{o}}$ & -3.795 \\
\hline & 4 & 8 & 2.1 & $a^{6} \mathrm{D}_{5 / 2}$ & ${ }^{6} \mathrm{P}_{5 / 2}^{\mathrm{o}}$ & -1.702 \\
\hline 516.77 & 8 & 8 & 2.17 & $a^{6} \mathrm{D}_{3 / 2}$ & $y^{6} \mathrm{P}_{3 / 2}^{\mathrm{o}}$ & -1.847 \\
\hline 37.75 & 4 & 5 & 2.1 & $a^{6} \mathrm{D}_{1 / 2}$ & $y^{6} \mathrm{P}_{3 / 2}^{\mathrm{o}}$ & -2.017 \\
\hline 13.49 & 32 & 6 & 3.0 & $60^{1 / 2}$ & $e^{6} \mathrm{~S}_{5 / 2}^{3 / 2}$ & -0.251 \\
\hline 016.64 & 32 & 5 & 3.06 & $z^{6} \mathrm{P}_{5 / 2}^{\mathrm{o}}$ & $e^{6} \mathrm{~S}_{5 / 2}$ & -0.216 \\
\hline 5021.79 & 32 & 6 & 3.06 & $z^{6} \mathrm{P}_{7 / 2}^{\mathrm{o}}$ & $e^{6} \mathrm{~S}_{5 / 2}$ & 0.034 \\
\hline
\end{tabular}

Notes. The three spectral lines below $4000 \AA$ are Mn II lines. The multiplet is indicated in the second column.

the basis of the close-coupling expansion of the wavefunction as

$\Psi(E ; S L \pi)=A \sum_{i} \chi_{i} \theta_{i}+\sum_{j} c_{j} \phi_{j}$

where $A$ is the anti-symmetrisation operator, $\chi_{i}$ is the target ion wavefunctions in the target state $(S L \pi)_{i}, \theta_{j}$ is the wavefunction for the free electron, and $\phi_{j}$ are short range correlation functions for the bound $\left(\mathrm{e}^{-}+\mathrm{ion}\right)$ system.

The calculations were done in LS-coupling and include all states with valence electron excitations up to the principal quantum number $n=10$. The single-electron orthogonal orbitals that represent the atomic structure of the $\mathrm{Mn}^{+}$target, were derived 


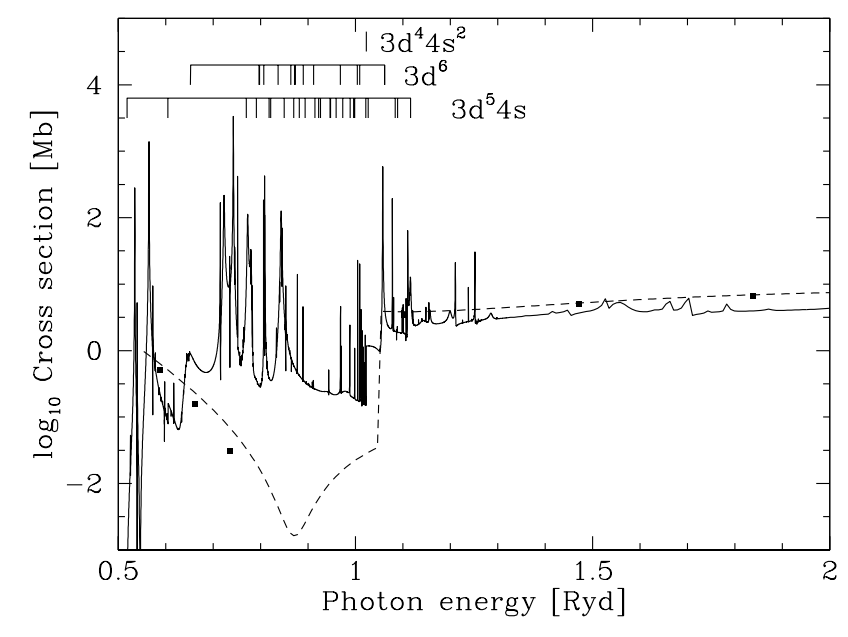

Fig. 2. Photo-ionisation cross-section of $3 \mathrm{~d}^{5} 4 \mathrm{~s}^{2}$ a ${ }^{6} \mathrm{~S}$ ground term of Mn I. The present R-matrix cross-section is depicted by the solid line. The central-field cross-sections of Verner \& Yakovlev (1995) and Reilman \& Manson (1979) are indicated by the dashed line and square dots, respectively. Above the figure we show the energies of all $3 d^{5} 4 s$, $3 \mathrm{~d}^{6}$, and $3 \mathrm{~d}^{4} 4 \mathrm{~s}^{2}$ thresholds.

using the AUTOSTRUCTURE code (Badnell 1997). The code employs scaled Thomas-Fermi-Dirac-Amaldi central-field potential. We adopted configuration interaction expansion with spectroscopic orbitals $1 \mathrm{~s}, 2 \mathrm{~s}, 2 \mathrm{p}, 3 \mathrm{~s}, 3 \mathrm{p} \mathrm{3d}, 4 \mathrm{~s}, 4 \mathrm{p}, 4 \mathrm{~d}, 5 \mathrm{~s}$, and $5 \mathrm{p}$. The configurations and scaling parameters for the orbitals are presented in Table B.2. LS terms of the target Mn II ion included in the close-coupling expansion are provided in Table B.1.

The cross-sections are sampled at 5000 evenly-spaced energy points between zero and 0.8 Ryd above the first ionisation threshold, followed by 250 points from 0.8 Ryd to $2.0 \mathrm{Ryd}$. This mesh is also preserved in our NLTE calculations with DETAIL and with MULTI2.3, such that all resonances are fully accounted for in the statistical equilibrium calculations. These resonances are mostly caused by the photo-excitation of the core and dominate the cross-sections of the majority of Mn I states. For the other levels, we employed hydrogenic cross-sections sampled on a regular mesh. The hydrogenic cross-sections were computed using the effective principal quantum number.

Figure 2 shows the total photo-ionisation cross-section for the ground state of Mn I, compared with the central field approximation results of Verner \& Yakovlev (1995) and Reilman \& Manson (1979). The close coupling expansion accounts for the photo-ionisation of the outer $4 \mathrm{~s}$ sub-shell, as well as the open inner $3 \mathrm{~d}$ sub-shell of the ground state $3 \mathrm{~d}^{5} 4 \mathrm{~s}^{2}{ }^{6} \mathrm{~S}$ of $\mathrm{Mn}$. The coupling of all relevant photo-ionisation channels results in extensive auto-ionisation structures and ensures that no sharp discontinuity in the cross-section at the $3 \mathrm{~d}$ inner-shell ionisation edge is present. By contrast, the central field approximation, which misses channel couplings, yields a cross-section that is severely underestimated up to the opening of the $3 \mathrm{~d}$ sub-shell, where a discontinuity appears. The importance of channel couplings in representing low-energy photo-ionisation cross-sections of ironpeak elements is well known (Bautista \& Pradhan 1995). For energies beyond the $3 \mathrm{~d}$ sub-shell, the central field cross-sections agree very well with our data, which gives additional confidence on the accuracy of our results. Figure A.1 also show the comparison of the cross-sections with the hydrogenic data. Clearly the differences are substantial and amount to several orders of magnitude in the background, but also all resonances that contribute to the over-ionisation at longer wavelengths are missing in the hydrogenic data.

DETAIL does not have a provision for including the partial ionisation channels to specific states of the target ion. Consequently, we adopted the total photo-ionisation crosssections, computed by adding the partial cross-sections for each Mn I state.

\subsubsection{Inelastic collisions with $\mathrm{H}$ atoms}

The rate coefficients for the bound-bound transitions in Mn I caused by collisions with $\mathrm{H}$ atoms, as well as for Mn II collisions with $\mathrm{H}^{-}$, were taken from Belyaev \& Voronov (2017c). We also computed new rates for these processes in this work.

The data from Belyaev \& Voronov (2017c) are available for the transitions between 19 levels $^{2}$ of $\mathrm{Mn} \mathrm{I}$ interacting with $\mathrm{H}$ and the ground state of Mn II interacting with $\mathrm{H}^{-}$. They represent collisional excitation, de-excitation, mutual neutralisation, and ion-pair formation ${ }^{3}$ due to the transitions between ${ }^{7} \Sigma^{+}$molecular states.

Here we present new calculations of the H I collision rates for 71 additional levels of $\mathrm{Mn} \mathrm{I}$ interacting with $\mathrm{H}$ and the first excited state of Mn II interacting with $\mathrm{H}^{-}$. The first excited ionic state of the MnH molecule has ${ }^{5} \Sigma^{+}$symmetry and only covalent molecular states of the same symmetry were considered in the non-adiabatic nuclear dynamical calculations. These states are listed in Table B.3.

All calculations were performed within the simplified quantum model (Belyaev \& Yakovleva 2017b,a), which allows the identification of a rate coefficient for a particular process using general dependences of the reduced rate coefficients on the electron binding energies. The binding energies are calculated from different ionic limits for the cases of non-adiabatic transitions between ${ }^{7} \Sigma^{+}$molecular states and between ${ }^{5} \Sigma^{+}$states. The rate coefficients for the excitation and de-excitation processes are summed over molecular symmetry, when the initial and the final state of the process have both ${ }^{7} \Sigma^{+}$and ${ }^{5} \Sigma^{+}$symmetries.

Neutralisation rate coefficients for collisions of $\mathrm{Mn}^{+}\left(3 \mathrm{~d}^{5} 4 \mathrm{~s}^{5} \mathrm{~S}\right)+\mathrm{H}^{-}$are presented in Fig. 3 as a function of the electron binding energy. For the case of the $\mathrm{MnH}$ collisional system involving quintet molecular states, the largest rate coefficients at $6000 \mathrm{~K}$ correspond to the mutual neutralisation processes at $\operatorname{Mn}\left(3 \mathrm{~d}^{5} 4 \mathrm{~s} 4 \mathrm{pu}^{6} \mathrm{P}^{\circ}\right)+\mathrm{H}, \quad \mathrm{Mn}\left(3 \mathrm{~d}^{5} 4 \mathrm{~s} 5 \mathrm{df} \mathrm{f}^{6} \mathrm{D}\right)+\mathrm{H}$, $\operatorname{Mn}\left(3 d^{5} 4 s 4 f w^{6} F^{\circ}\right)+H, \quad M n\left(3 d^{5} 4 s 6 \mathrm{pt}^{6} \mathrm{P}^{\circ}\right)+\mathrm{H}$ states, having values $\sim 6 \times 10^{-8} \mathrm{~cm}^{3} \mathrm{~s}^{-1}$. The rate coefficients for the (de-)excitation processes are, at least, one order of magnitude lower than the rates for the neutralisation and ion-pair formation processes, as found in previous calculations for other chemical elements (Belyaev et al. 2014, 2017; Yakovleva et al. 2017).

We also derive new rate coefficients for the 42 levels of $\mathrm{Mn}$ II interacting with $\mathrm{H}$ and for the ground state of Mn III interacting with $\mathrm{H}^{-}$. These calculations were performed for the transitions between ${ }^{6} \Sigma^{+}$molecular states as the ionic state of $\mathrm{MnH}^{+}$has ${ }^{6} \Sigma^{+}$symmetry. The states are presented in Table B.4. Neutralisation rate coefficients for collisions $\mathrm{Mn}^{2+}\left(3 \mathrm{~d}^{56} \mathrm{~S}\right)+\mathrm{H}^{-}$ as a function of the electron bound energy are shown in Fig. 4. The largest rate coefficient for the case of $\mathrm{MnH}^{+}$collisions at $6000 \mathrm{~K}$ with the value of $7.5 \times 10^{-8} \mathrm{~cm}^{3} \mathrm{~s}^{-1}$ corresponds

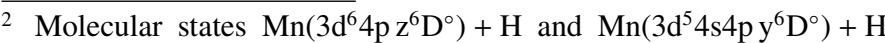
are excluded from the calculations because they have ${ }^{7} \Sigma^{-}$symmetry, while other considered states have ${ }^{7} \Sigma^{+}$symmetry.

3 We emphasise that all these processes are bound-bound transitions, in which electrons remain bound to $\mathrm{Mn}$ or to $\mathrm{H}$ atoms, and are referred to this way in the text.
} 


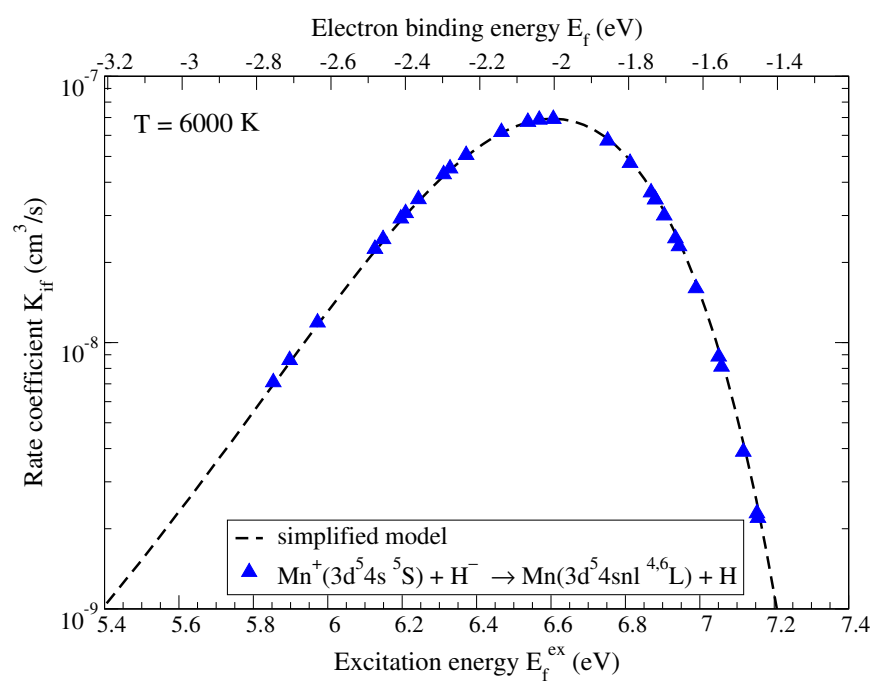

Fig. 3. Neutralisation rate coefficients for $\mathrm{Mn}^{+}\left(3 \mathrm{~d}^{5} 4 \mathrm{~s}^{5} \mathrm{~S}\right)+\mathrm{H}^{-}$collisions as a function of electronic energy in different excited states of $\mathrm{Mn}$ I. The dashed line represents the reduced rate coefficient given by the simplified model.

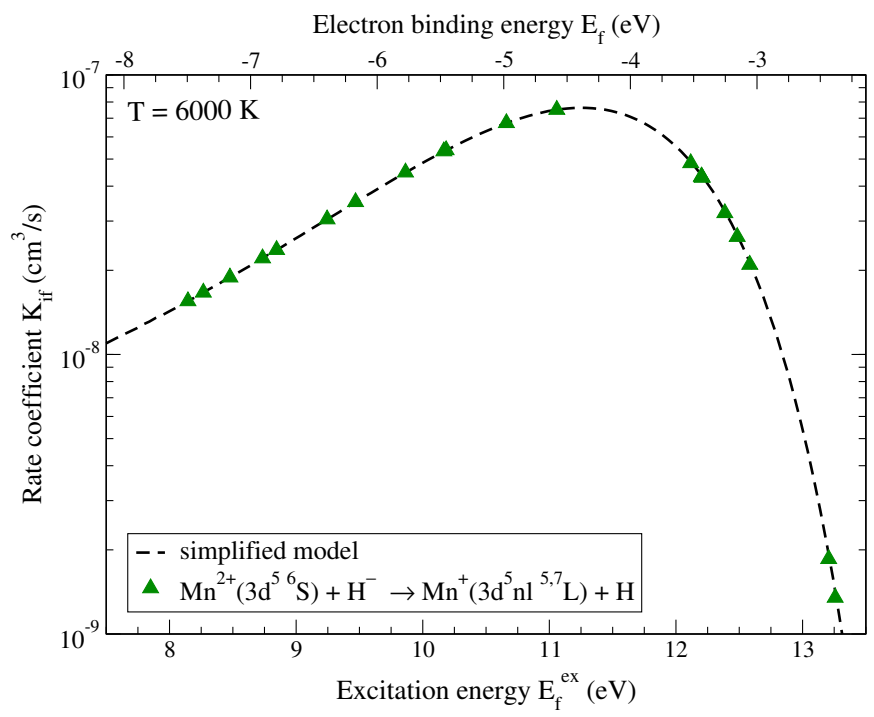

Fig. 4. Neutralisation rate coefficients for $\mathrm{Mn}^{2+}\left(3 \mathrm{~d}^{5}{ }^{6} \mathrm{~S}\right)+\mathrm{H}^{-}$collisions as a function of electronic energy in different excited states of Mn II. The dashed line represents the reduced rate coefficient given by the simplified model.

to the mutual neutralisation process $\mathrm{Mn}^{2+}\left(3 \mathrm{~d}^{56} \mathrm{~S}\right)+\mathrm{H}^{-} \rightarrow$ $\mathrm{Mn}^{+}\left(3 \mathrm{~d}^{5} 5 \mathrm{p} \mathrm{v}^{5} \mathrm{P}^{\circ}\right)+\mathrm{H}$.

Our data apply to $J$-averaged energy states, but the NLTE model atom includes fine structure. We have tested different recipes that are used in the literature to deal with this case. In particular, Barklem (2007) propose to divide the rate coefficient by the number of the target states. However, we found that the effect of distributing the collision rate coefficients across the target states is virtually null. In particular, for the high-excitation Mn I lines in the model of a metal-poor dwarf, this leads to an error in the line equivalent width of less than $0.1 \%$, which is negligibly small for abundance determinations. We, hence, assigned the same rate coefficient for each fine structure level of a given term ${ }^{4}$. This is analogous to our handling of the photo-ionisation

4 Both Mn model atoms, with and without distributed rates, can be obtained from the corresponding author by request. data, which are also provided for a given term. The new rate coefficients are available in the supplementary material.

In the model atom, we tabulated the rates of exothermic processes for the bound-bound reactions, meaning the transitions accompanied by the release of energy $\left(E_{j}>E_{i}\right.$, where the transition occurs from the higher energy level $j$ to the lower energy level $i$ ). These rates are almost independent of temperature, which minimises interpolation errors. For the charge transfer, the reverse is true, hence we tabulated the rates of endothermic processes. The reverse rates are computed from the detailed balance internally within the code, see Eq. (5) in Belyaev \& Voronov $2017 \mathrm{c}$ (note that neither $n_{\mathrm{H}}$ nor $n_{\mathrm{H}^{-}}$explicitly enter these equations):

$r_{i j}=r_{j i} \frac{g_{j}}{g_{i}} \exp \frac{-\Delta E_{j i}}{k_{\mathrm{B}} T}$,

where $r_{i j}$ and $r_{j i}$ are rate coefficients for the transitions between $i$ and $j ; g_{i}$ and $g_{j}$ the statistical weights of these levels; and $E_{j i}$ the energy difference ("energy defect") between the energies of the two states.

Apart from the avoided crossing mechanism, one can estimate additional rate coefficients using the free electron model, which is expected to include other inelastic mechanisms except the long-range ionic covalent mechanism (Barklem 2016; Amarsi et al. 2018c, 2019). We hence also supplemented the model atom with collision rates for all Mn I states computed using the scattering-length approximation ${ }^{5}$ according to Eq. (18) of Kaulakys (1991) using the code developed by Barklem (2017). As described by Osorio et al. (2015) and Barklem (2016), the rate coefficients computed in this way need to be redistributed over all possible final spin states. Here, we assume that all transitions have two possible final spin states, and that each final spin state is equally likely, so that the rate coefficients were reduced by a factor of two. The error associated with this assumption is less than a factor of two. The Kaulakys model is developed for application to Rydberg molecular states, hence, our implementation shall be viewed as a limiting case with strong collisional binding, and, hence, might underestimate NLTE effects.

In Fig. 5 we compare the new $\mathrm{H}$ collision rates with the data computed using the Steenbock \& Holweger (1984) formulation of the classic theory developed by Drawin $(1968,1969)$. The classic formalism does not cover the mutual neutralisation and ion-pair formation processes. The differences in the $\mathrm{H}$ excitation rates for the individual energy levels amount up to 7 orders of magnitude in both directions. The overall distributions as a function of energy difference have similar shapes, with the largest rate coefficients for the transitions between nearby energy levels. The rate coefficients for the charge transfer reactions are also qualitatively similar to the Drawin's bound-free recipe, which describes collisional ionisation, but quantitatively there are major differences of up to 5 orders of magnitude. The quantum-mechanical charge transfer rates are typically lower for the neutral species, whereas for the singly-ionised species these are larger than the Drawin's rates. The Kaulakys rates are typically very low for the low-energy levels, but gradually increase closer to the ionisation threshold and thus somewhat compensate the downturn in the quantum-mechanical data, leading to higher collisional thermalisation. In Sect. 4.1.2 we briefly report on how this impacts the line profiles and the NLTE abundance corrections.

5 These data were calculated with assistance from Anish Amarsi and Paul Barklem. 

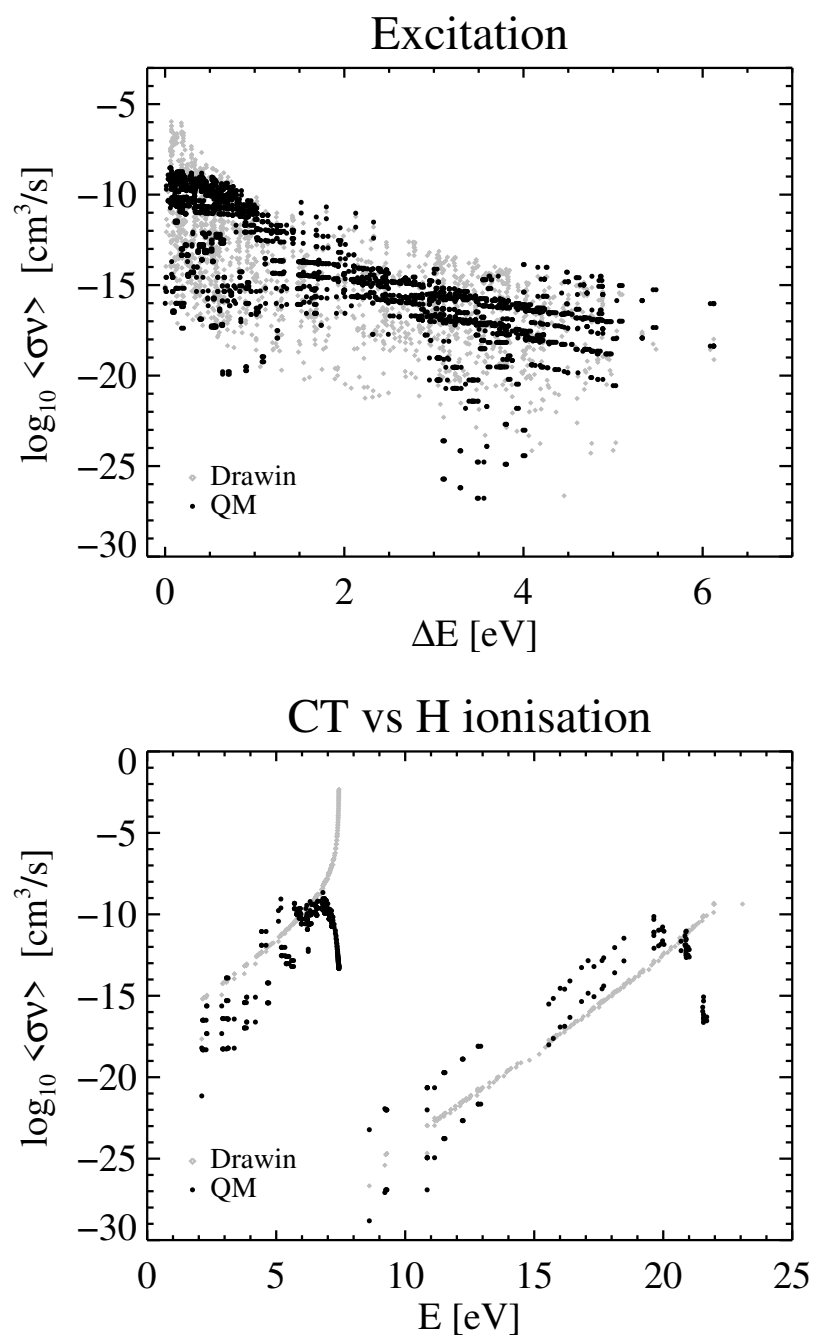

Fig. 5. Excitation (top panel) and ion-pair formation (bottom panel, CT stands for "charge transfer") rate coefficients for Mn I and Mn II as used in this work and compared to Drawin's formulae (Drawin 1968, 1969; Steenbock \& Holweger 1984).

\subsection{Model atmospheres}

As in our previous papers, we used MARCS (Gustafsson et al. 2008) and MAFAGS-OS (Grupp 2004a,b) model atmosphere grids. These are 1D LTE model atmospheres, with certain differences regarding the treatment of convective energy transport (mixing length), opacity, and the solar abundance mixture. The depth discretisation and the vertical extent of the models are also slightly different, as MAFAGS-OS covers the range from -6 to +2 in $\log \tau_{5000}$, whereas the MARCS models sample the Rosseland optical depths from -4 to +2 . Nonetheless, the thermodynamic structures of the models for the given input parameters are very similar (Bergemann et al. 2012, 2017).

The 3D model atmospheres are taken from the STAGGER model atmosphere grid (Collet et al. 2011; Magic et al. 2013) and computed with the STAGGER code (Nordlund \& Galsgaard 1995). A 3D model consists of a series of computational boxes that represent a time series, which are referred to as snapshots. These snapshots are selected from a larger time series of snapshots that are produced from the STAGGER code and are selected at a time when they have reached dynamical and thermal relaxation. For our purpose and for the sake of time, we have chosen to work with five snapshots. Importantly, and unlike an equivalent
1D model, 3D models provide $x, y$, and $z$ velocity fields for every voxel. This means that post-processing spectrum synthesis code provides parameter-free description of Doppler broadening, including asymmetric line profiles, which trace these gas flows at each voxel.

Figure 6 depicts the 3D temperature structures (blue 2D histogram) in a representative snapshot for four benchmark stars, along with the 1D MARCS (solid red line) and $\langle 3 \mathrm{D}\rangle$ (dashed grey line) profiles. The average temperature of the full 3D model and the 1D hydrostatic model are fairly different in the outermost regions of the atmosphere, as seen by comparing the 1D hydrostatic models with the $\langle 3 \mathrm{D}\rangle$ models. In particular, in the outer layers of metal-poor models the $1 \mathrm{D}$ hydrostatic models are significantly hotter (up to $500 \mathrm{~K}$ ) compared to the 3D structures (see also Bergemann et al. 2017). Also the models diverge in deeper regions of the models, where the continuum usually forms. This is mostly due to the treatment of convection between the $1 \mathrm{D}$ and 3D model atmospheres.

The average temperature structure of the 1D model of the metal-poor RGB star HD 122563 is not very different from its $\langle 3 \mathrm{D}\rangle$ counterpart. Our adopted MARCS models are taken from Bergemann et al. (2012, their Fig. 1). We explore the impact of an adopted 1D model in Sect. 4.6, by performing the abundance analysis with a MAFAGS-OS suit of models. We also note that line formation is not only sensitive to the mean $T(\tau)$ and $P(\tau)$ structures, but also to the horizontal inhomogeneities. The latter play a significant role in the abundance analysis.

The scope of this paper is limited to the analysis of a small sample of 3D models, including that of the Sun, a typical dwarf, and a typical giant (Table 2). We also include tailored 3D models computed for the parameters of the benchmark metal-poor stars HD 122563, HD 140283, and HD 84937 (Sect. 2). To make the NLTE radiative transfer problem computationally tractable, we have to resample the full 3D model cubes onto a less fine, yet equidistant, grid in horizontal coordinates. However, we test the effect of the resolution of the cube for radiative transfer in Sect. 4.2 and find virtually no differences in the resulting atomic number densities and line profiles for horizontal resolutions of $(x, y, z)=30,30,230$ and the original cubes $((x, y, z)$ $=240,240,230)$. Hence, the former is taken to be the default resolution for most of the analysis presented in this work. We solve the 3D NLTE radiative transfer problem for a set of snapshots for each of the 3D model atmospheres listed in Table 2. These snapshots are extracted at regular time intervals from the full simulation that covers roughly two convective turnover timescales (Collet et al. 2011; Magic et al. 2013). The convergence criterion, that is the maximum relative correction in the population numbers, $\max |\delta N / N|$, is set to $10^{-3}$. This is fully sufficient according to our experience with 1D NLTE radiative transfer.

\subsection{Statistical equilibrium}

We used two different codes to compute the SE of Mn. One is MULTI2.3 (Carlsson 1992), the other code is DETAIL (Butler $\&$ Giddings 1985). The codes solve the equations of radiative transfer and SE assuming a 1D geometry. The assumption of trace elements is used, that is, the element that is modelled in $\mathrm{SE}$ is assumed to have no effect on the model atmosphere. This is a good assumption for $\mathrm{Mn}$ as it is not an electron donor, nor does it have a high impact on the overall opacity. Both codes adopt the accelerated lambda iteration (ALI) technique and the operator acting on the source function.

The basic differences between the codes are described in Bergemann et al. (2012). The tests described in the following 
M. Bergemann et al.: Observational constraints on the origin of the elements. I.

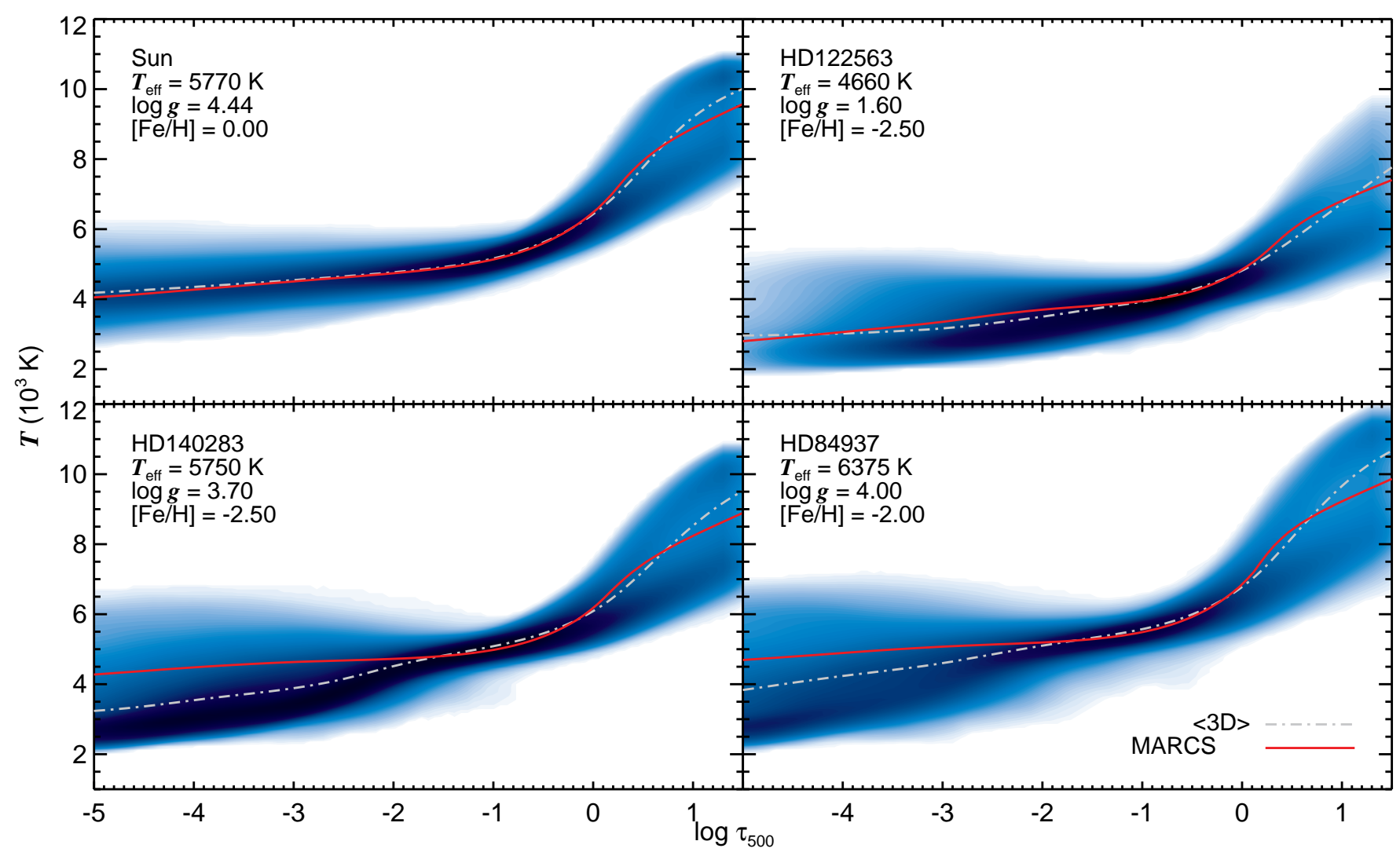

Fig. 6. 3D, 1D, and $\langle 3 \mathrm{D}\rangle$ temperature structures as a function of Rosseland optical depth for benchmark stars. The blue-shaded regions indicate the kinetic temperature distributions in the representative snapshot from the 3D convection simulations. The stellar parameters are given in the inset. We note that $\langle 3 \mathrm{D}\rangle$ models are provided only to illustrate the difference between the average structure of the $1 \mathrm{D}$ and 3D models, however, the $\langle 3 \mathrm{D}\rangle$ are not used in our abundance analysis.

Table 2. Parameters of 3D convective and 1D hydrostatic model atmospheres.

\begin{tabular}{llccc}
\hline \hline No. & Name & $\begin{array}{c}T_{\text {eff }} \\
\mathrm{K}\end{array}$ & $\begin{array}{c}\log g \\
\mathrm{dex}\end{array}$ & $\begin{array}{c}{[\mathrm{Fe} / \mathrm{H}]} \\
\mathrm{dex}\end{array}$ \\
\hline 1 & Sun & 5777 & 4.4 & 0.0 \\
2 & HD 84937 & 6400 & 4.0 & -2.0 \\
3 & HD 140283 & 5750 & 3.7 & -2.5 \\
4 & HD 122563 & 4600 & 1.6 & -2.5 \\
\hline 5 & Sub-solar metallicity dwarf & 6500 & 4.0 & -1.0 \\
6 & Metal-poor dwarf & 6500 & 4.0 & -2.0 \\
7 & Sub-solar metallicity giant & 4500 & 2.0 & -1.0 \\
8 & Metal-poor giant & 4500 & 2.0 & -2.0 \\
\hline
\end{tabular}

Notes. In the 1D LTE radiative transfer calculations with MARCS models, we assume $\xi_{\mathrm{t}}=1$.

sections will be performed imposing the same input conditions (LTE populations) and the same model atmospheres, in order to maximise the consistency. The main difference between the codes are in the handling of thermodynamic parameters and of the background opacities. In particular, DETAIL takes the partial pressures and partition functions from the input model atmosphere, whereas MULTI2.3 includes a package to compute these parameters given the input structures $T(\tau)$ and $P_{\mathrm{e}}(\tau)$ as a function of optical depth or column mass.

In order to maximise the homogeneity of the analysis, we have also computed background opacity tables for MULTI2.3 using the updated line lists from DETAIL (Bergemann et al. 2015). The MARCS (Gustafsson et al. 2008) and Turbospectrum (Plez 2012) codes were used to generate a table of opacities for a set of temperature and pressure points at more than $10^{5}$ wavelengths, $\mathrm{Mn}^{6}$ lines being omitted. This table is then interpolated by MULTI2.3 to produce detailed line background opacities.

All calculations with MULTI2.3 are carried out by simultaneously solving the intensity at all angles, using the Feautrier method with all scattering terms included consistently (the ISCAT option set to 1). This is important when scattering in the background opacity is significant, as is the case in the Wien regime. We have tested the line formation disabling this option, but found that this has a very strong effect on the blue and UV lines of Mn I and Mn II, significantly over-estimating the line abundances, because of reduced continuum intensities.

MULTI3D is an MPI-parallelised, domain-decomposed NLTE radiative transfer code that solves the equations of radiative transfer in 3D geometry using the ALI method. The formal solution of radiative transfer is done via the short characteristics method (Kunasz \& Auer 1988) that solves the integral form of the radiative transfer equation across one subdomain per time step. Carlson (1963)'s A4 quadrature is employed to compute the angle-averaged radiation field in the SE solution. The approximate operator is constructed using the formulation developed by Rybicki \& Hummer (1991, 1992), where only the diagonal of the full $\Lambda$ operator is used (for discussion of this approximation, see, e.g. Bjørgen \& Leenaarts 2017).

6 Note that also Ba lines were omitted, in order to use these opacity tables for the 2 nd paper in the series by Gallagher et al. (in prep.). 
MULTI3D will accept three types of 3D model atmosphere formats, including the commonly used Bifrost (Gudiksen et al. 2011) and STAGGER models (Magic et al. 2013). The code will also accept any 3D model providing the temperature, density, electron number density, and $x, y$, and $z$ velocity fields are supplied on a Cartesian grid that is both horizontally periodic and equidistantly spaced. The code can compute radiative transfer using the $1.5 \mathrm{D}$ approximation, which treats each column of grid points in a model as a separate plane-parallel atmosphere, or using full $3 \mathrm{D}$ radiative transfer. The rate equations are assumed to be time-independent and the advection term is not included. For more information about the code, we refer to Leenaarts et al. (2012) and Bjørgen \& Leenaarts (2017).

\section{Results}

We begin the discussion of results with a brief account of NLTE effects in Mn (Sect. 4.1). The key properties of the statistical equilibrium of Mn are summarised in Sect. 4.1.1. 1D NLTE abundance corrections across a large parameter space are presented in Sect. 4.1.2. Line formation and abundances determined using 3D inhomogeneous atmospheres are the subjects of subsequent sections. Section 4.2.1 deals with the properties of line formation in full 3D solar simulation cubes, that we refer to as photon kinematics. In Sect. 4.2.2, we discuss simplified radiative transfer models and explore how these impact the line profiles compared to the full 3D NLTE solution. The results of the 3D NLTE solar abundance analysis are presented in Sect. 4.3. In Sect. 4.4, we discuss the impact of 3D NLTE on the line profiles, on their equivalent widths (EW), and on the abundance diagnostic for four metal-poor models. The results of LTE and NLTE calculations with 3D models are discussed in Sect. 4.5. Finally, in Sect. 4.6, we use 3D convective models of the benchmark metal-poor stars HD 84937, HD 140383, and HD 122563 to derive 3D NLTE abundances.

\section{1. $1 D$ NLTE}

\subsubsection{Departures from LTE}

This work does not deal extensively with the properties of statistical equilibrium of Mn nor with the details of line formation, as this has been discussed in great detail in our previous work (Bergemann \& Gehren 2007). Additionally Bergemann \& Gehren (2008) address the details of line transfer in metal-poor stars. It suffices to remind the reader that $\mathrm{Mn}$, similar to other Fe-group elements, is a photo-ionisation dominated ion. Simply stated, the large photo-ioinisation cross-sections of Mn I energy levels imply significant over-ionisation in the atmospheres of cool stars in the more general SE case compared to LTE. Mn I becomes significantly over-ionised (compared to LTE) in metalpoor or in hotter stellar atmospheres due to their strong UV radiation fields. The effect of the radiation field is furthermore amplified in the atmospheres of giants owing to their lower densities and hence, less efficient collisional processes.

The effect of the radiation field is reflected in the behaviour of Mn I level departure coefficients, $b_{i}$, which describe the ratio between NLTE and LTE atomic number densities. Departures from LTE take place in the line formation layers. Figure 7 shows that for Mn I, this ratio is typically $\lesssim 1$, as in NLTE the fraction of atoms in a given energy state is less than that predicted by the Saha-Boltzmann formulae. The departure coefficients of the ionic levels, Mn II, are very close to unity for the lower-lying energy levels, but deviate from thermal for the levels of higher excitation energy, $E \gtrsim 1 \mathrm{eV}$.
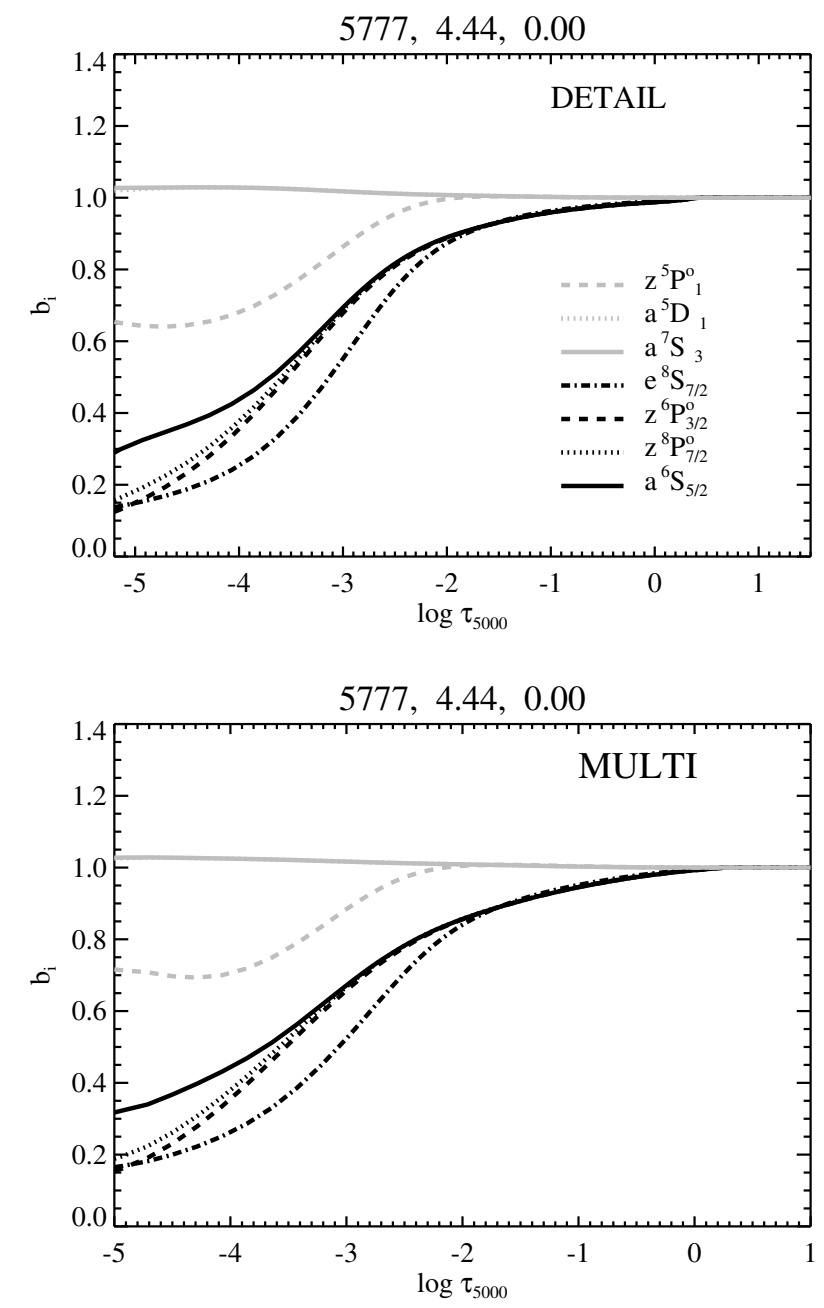

Fig. 7. Mn departure coefficients for solar MARCS model atmosphere as a function of optical depth at $5000 \AA$, computed using DETAIL (top panel) and MULTI (bottom panel) codes. The surface parameters of the Sun, $\left(T_{\text {eff }}, \log g\right.$, and $\left.[\mathrm{Fe} / \mathrm{H}]\right)$ are given in the figure details.

Figure 7 shows that the departures from NLTE in the lineformation region, $-2 \lesssim \log \tau_{5000} \lesssim 0$, are moderate in the solar atmosphere, but only slightly smaller than our previous estimates in Bergemann \& Gehren (2007). The differences with respect to the latter study are caused by the use of new quantummechanical photo-ionisation rates and $\mathrm{H}$ collision rates, as well as the implementation of fine structure for most of the Mn I levels. Collisions with electrons are not important in the physical conditions of the solar atmosphere. On the other hand, inelastic collisions with $\mathrm{H}$ atoms have a non-negligible effect on the atomic level populations and significantly contribute to the overall thermalisation of the system. This effect is not linear and may increase or decrease the departures from LTE for individual energy levels, and hence spectral lines, depending on the $T_{\text {eff }}$, $\log g$, and metallicity of a star.

The comparison of the $b_{i}$ profiles computed using DETAIL and MULTI2.3 (Fig. 7) suggests that the codes are consistent, given the same input conditions, such as the model atom, line opacities, and model atmospheres. MULTI2.3 predicts slightly larger departures from LTE compared to DETAIL. This has also been shown in our earlier paper for Fe (Bergemann et al. 2012), and is likely related to continuum opacities and/or the numerical implementation of the coupled SE and radiative transfer equations. 
The behaviour of departure coefficients is very different in the model atmosphere of a metal-poor red giant star (Fig. 8). All Mn I levels show a stronger under-population compared to the solar model, implying larger differences between LTE and NLTE abundances in metal-poor stars. The energy levels of the Mn II lines are also affected by NLTE. In particular, the levels of the upper term $z^{5} \mathrm{P}^{\mathrm{o}}$ experience overpopulation caused by the radiative pumping in nine strong near-UV lines of Mn II multiplet Nr. $31\left(a^{5} \mathrm{D}-z^{5} \mathrm{P}^{\mathrm{o}}\right)$ in the deeper layers. However, the levels of $z^{5} \mathrm{P}^{\mathrm{o}}$ become under-populated at $\log \tau_{5000} \sim-1.5$, as these lines progressively become optically thin. Consequently, one would expect significant NLTE effects on the formation of Mn II lines, largely driven by the changes in the line source function itself. Comparing the departure coefficients computed using the old atom from Bergemann \& Gehren (2008) (Fig. 8, top panel) and the new atom in this work (Fig. 8, middle panel), we find substantial differences. The influence of new quantum-mechanical collisions with hydrogen is admittedly greater in the new atom, despite the larger photo-ionisation cross-sections. On the other hand, contrasting the results obtained using the DETAIL code with MULTI2.3 (Fig. 8, middle and bottom panels) confirms that, similar to the Sun (see Fig. 7), the two codes produce quantitatively similar outputs. In the outer layers, the departures are slightly different, which could be related to the differences in the outer boundary conditions.

\subsubsection{NLTE abundance corrections}

An NLTE abundance correction is the quantity that is commonly used in stellar abundance studies to correct the abundances derived under the assumption of LTE. The abundance correction is defined as $\Delta=A$ (NLTE) $-\mathrm{A}(\mathrm{LTE})$, that is the difference in abundance required to fit a given spectral line assuming 1D LTE or 1D NLTE. We also employ this concept in our 3D NLTE analysis in Sect. 4.2.

Figure 9 illustrates the NLTE abundance correction for several metal-poor models in the metallicity range -3 to 0 . We only plot selected lines to illustrate the key results: the $3488 \AA$ line of Mn II, multiplet 4 (4030, 4033, $4034 \AA$ ) ), multiplet 18 (4783, $4823 \AA)$, and multiplet $32(6013,6016,6021 \AA)$ of Mn I. The behaviour of abundance corrections within a given multiplet is very similar, hence the lines are grouped by multiplets. The correction is not tailored to any particular star and is computed assuming the reference NLTE [Mn/Fe] of zero. We also explored the abundance corrections computed using LTE $[\mathrm{Mn} / \mathrm{Fe}]$ abundance of -0.5 to -0.8 , as it would be typically measured assuming LTE in metal-poor stars and found no significant differences in the corrections. The individual curves represent three possible scenarios that differ in the completeness of the model atom.

In Fig. 9, we illustrate the sensitivity of the corrections to the quantum-mechanical $\mathrm{H}$ data. The $\mathrm{CH}$ case (note that $\mathrm{CH}$ is not an abbreviation) corresponds to the model atom, which is devoid of quantum-mechanical H-collisional excitation processes (but includes charge transfer). The $\mathrm{CH} 0$ model lacks charge transfer processes, but includes collisional excitation. These two cases are compared to the model, which has both excitation and charge transfer $(\mathrm{CH} / \mathrm{CH} 0$ included). None of the three cases presented in this figure includes the Kaulakys (1985) collision rates.

The $\mathrm{H}$ collisions clearly have a different impact on the line formation in the atmospheres of giants and dwarfs. Based on the NLTE abundance corrections in the figures, it appears that for dwarfs, $\mathrm{H}$ collisions serve as a thermalising agent, decreasing the difference between NLTE and LTE. In the atmospheres of red giants, the effect is somewhat counter-intuitive: the lines
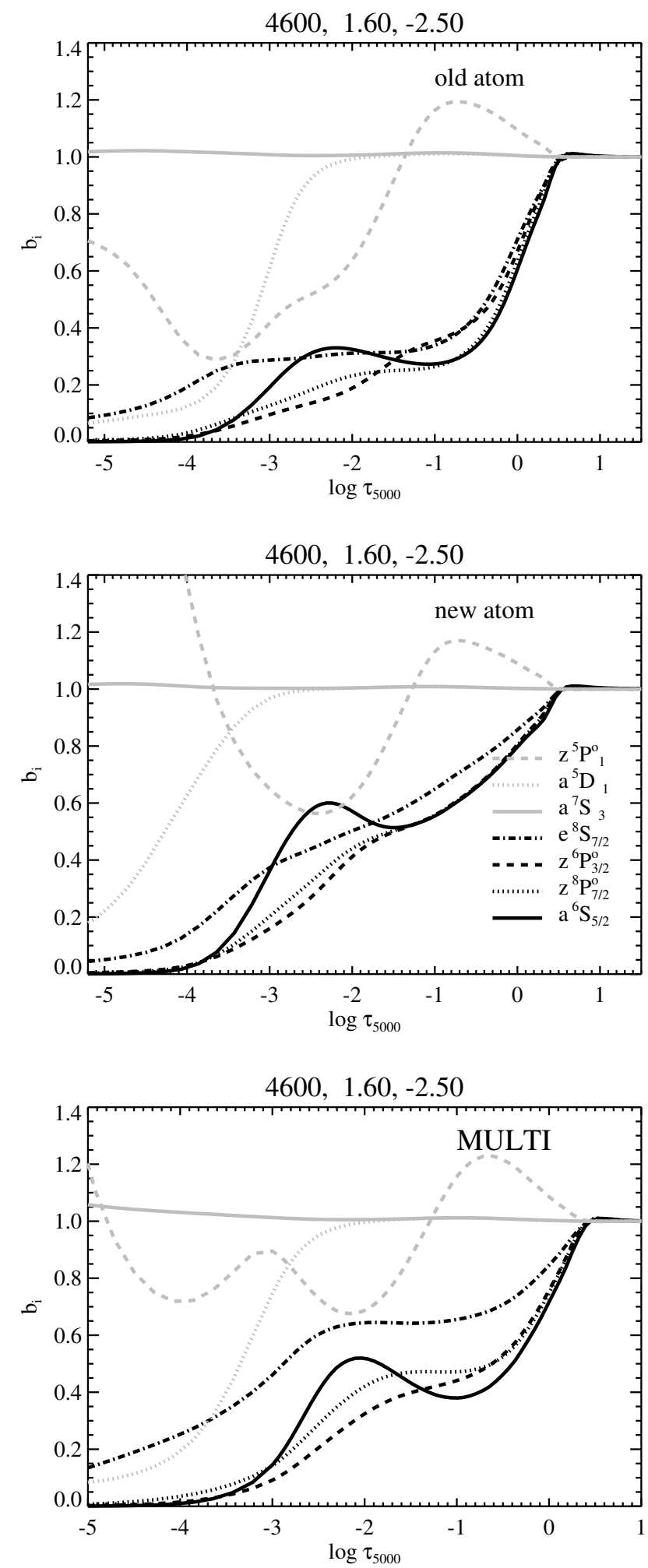

Fig. 8. Mn departure coefficients for model atmosphere of a metalpoor red giant. These were computed using the old $\mathrm{Mn}$ atom from Bergemann \& Gehren (2007) (top) and the new atom from this paper (middle panel: DETAIL, bottom panel: MULTI). The stellar parameters $\left(T_{\text {eff }}, \log g\right.$, and $\left.[\mathrm{Fe} / \mathrm{H}]\right)$ are given in the figure titles.

of multiplet 32 (6013-6021 $\AA$ triplet) have smaller NLTE corrections when $\mathrm{H}$ collisions are excluded. In fact, this is the indirect effect of over-ionisation, which is more efficiently transferred to higher-excitation states by collisions with $\mathrm{H}$. On the 


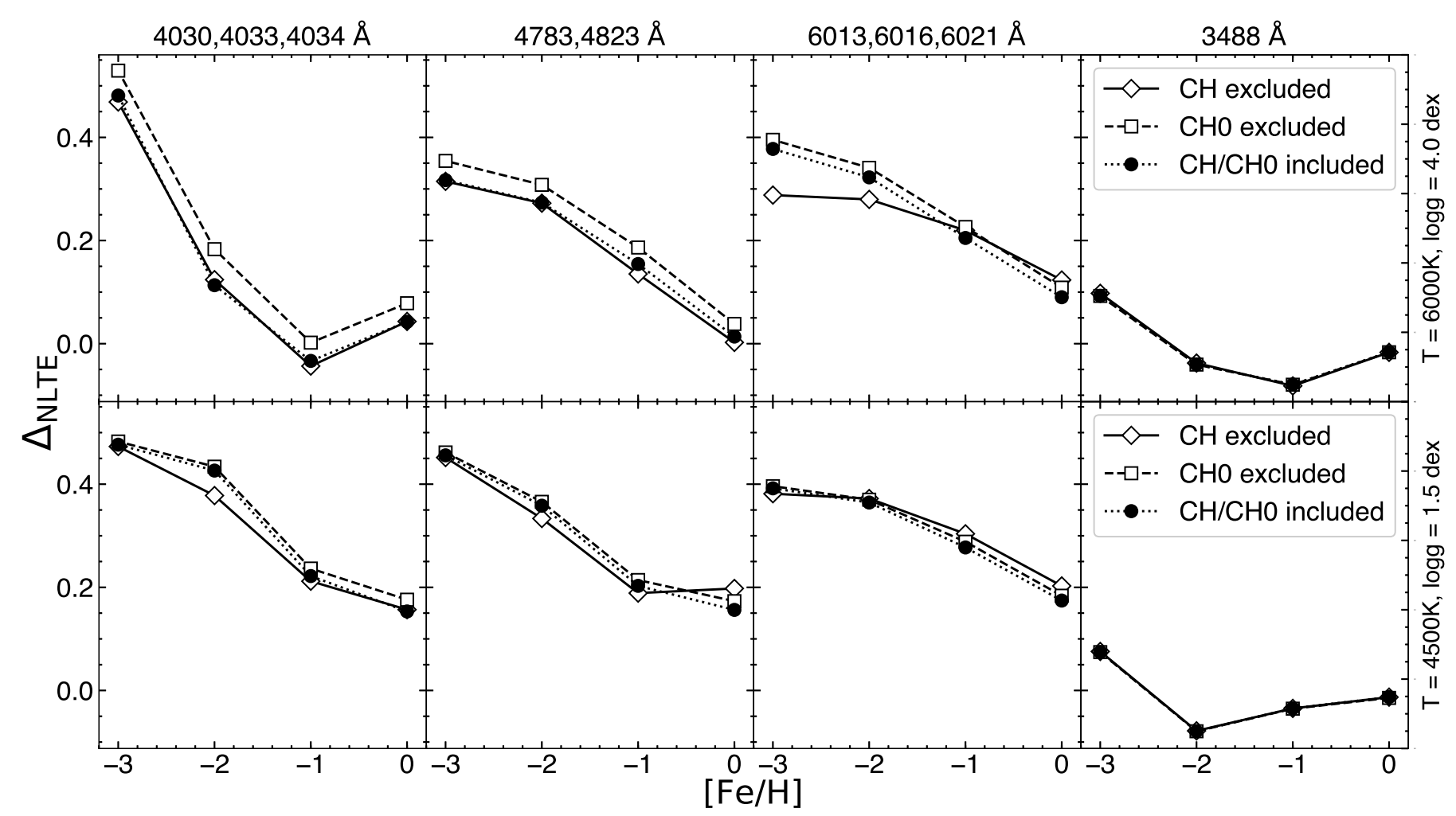

Fig. 9. NLTE abundance corrections for Mn I (4030, 4033, 4034, 4783, 4823, 6013, 6016, and $6021 \AA$ and Mn II (3488 $⿱$ ) lines. These are computed for a small grid of MARCS model atmospheres representative of dwarfs: $T_{\text {eff }}=6000, \log g=4.0$ (top panels), and red giants: $T_{\text {eff }}=4500, \log g=1.5$ (bottom panels) for a range of metallicities from 0 to -3 dex. Different curves represent the corrections derived using the model atoms with reduced complexity: (a) $\mathrm{CH}$ excluded, ignoring the excitation processes by collisions with $\mathrm{H}$ atoms; (b) $\mathrm{CH} 0$ excluded, ignoring the charge transfer reactions; and (c) $\mathrm{CH} / \mathrm{CHO}$ included with excitation and $\mathrm{CT}$ rates from the quantum-mechanical calculations. None of the three models (a-c) include the Kaulakys recipe.

other hand, the lines of multiplet 18 (4783, $4823 \AA)$ behave as expected from the simple considerations of increased rate of collisional thermalisation. Figure 9 also suggests that charge transfer (CT) reactions are more important in the atmospheres of dwarfs. Neglecting CT fully typically leads to abundance corrections being over-estimated by 0.05 dex for the models with $[\mathrm{Fe} / \mathrm{H}] \gtrsim-3$.

Figure 10 illustrates the influence of collision rates computed using the Kaulakys (1985) recipe. The reference atom includes the Kaulakys (1985) collisions in addition to the data from Belyaev et al. (2017), and we compare this model with the model that is devoid of the Kaulakys data. The differences with our reference model atom are modest, and do not exceed 0.05 dex for the dominant part of the parameter space. The effects that are possibly most significant occur when we neglect Kaulakys (1985) collisions. This leads to slightly over-estimated NLTE abundance corrections for the multiplet $32 \mathrm{Mn}$ I lines (6013 $6021 \AA$ triplet) in the RGB models. On the other hand, the NLTE abundance corrections for these very high-excitation lines are systematically under-estimated in the model of a dwarf.

The general picture is that the NLTE abundance corrections for the Mn I lines are positive and increase with decreasing metallicity, supporting our earlier study of Mn (Bergemann \& Gehren 2007, 2008) and of other similar ions (e.g. Fe, Bergemann et al. 2012; Lind et al. 2012). The corrections are slightly larger for the RGB model, especially at lower $[\mathrm{Fe} / \mathrm{H}]$. The higher-excitation lines, such as those of multiplets 18 and 32 are more sensitive to NLTE. Their NLTE abundance corrections typically increase with decreasing metallicity, but this trend slightly flattens below $[\mathrm{Fe} / \mathrm{H}] \sim-2$. Another noteworthy feature of these diagrams is the fact that the Mn II lines are also not immune to NLTE. It has been often assumed in the literature that the lines of ionic species do not show NLTE effects. The few strong excited lines of Mn II at $1.85 \mathrm{eV}$ show the classical NLTE effect of photon loss. This effect is small, but shows in the atmospheres of dwarfs and giants (see Fig. 10). It implies that lower abundances would be obtained from Mn II line, especially for the metal-poor stars with $[\mathrm{Fe} / \mathrm{H}]=-2$. At lower metallicity, the Mn II lines become sufficiently weak and radiative pumping effects dominate, leading to positive NLTE abundance corrections.

It is interesting, and it possibly presents the main difference with respect to our earlier study, that the strong resonance triplet of Mn I at 4030-4034 $\AA$ and the excited lines show very similar NLTE abundance corrections. The NLTE corrections exceed just about 0.4 dex in the atmospheres of RGB stars with $[\mathrm{Fe} / \mathrm{H}]=-3$. This is important, as LTE abundances derived from the resonance Mn I lines are known to be significantly lower compared to high-excitation Mn I features (Bonifacio et al. 2009; Sneden et al. 2016). Compared to the high-excitation features, in the 1D NLTE analysis there is no room for differentially larger NLTE corrections for the 4030-4034 $\AA$ triplet lines. In our previous work, a higher degree of over-ionisation in $\mathrm{Mn} \mathrm{I}$, and, in particular, over-ionisation from the ground state, was achieved by employing a tailored $S_{\mathrm{H}}$ scaling factor to the Drawin collisional (excitation and ionisation) $\mathrm{Mn} \mathrm{I}+\mathrm{H}$ I rates. As a consequence, the NLTE abundance corrections for the resonance triplet lines were significantly higher. 


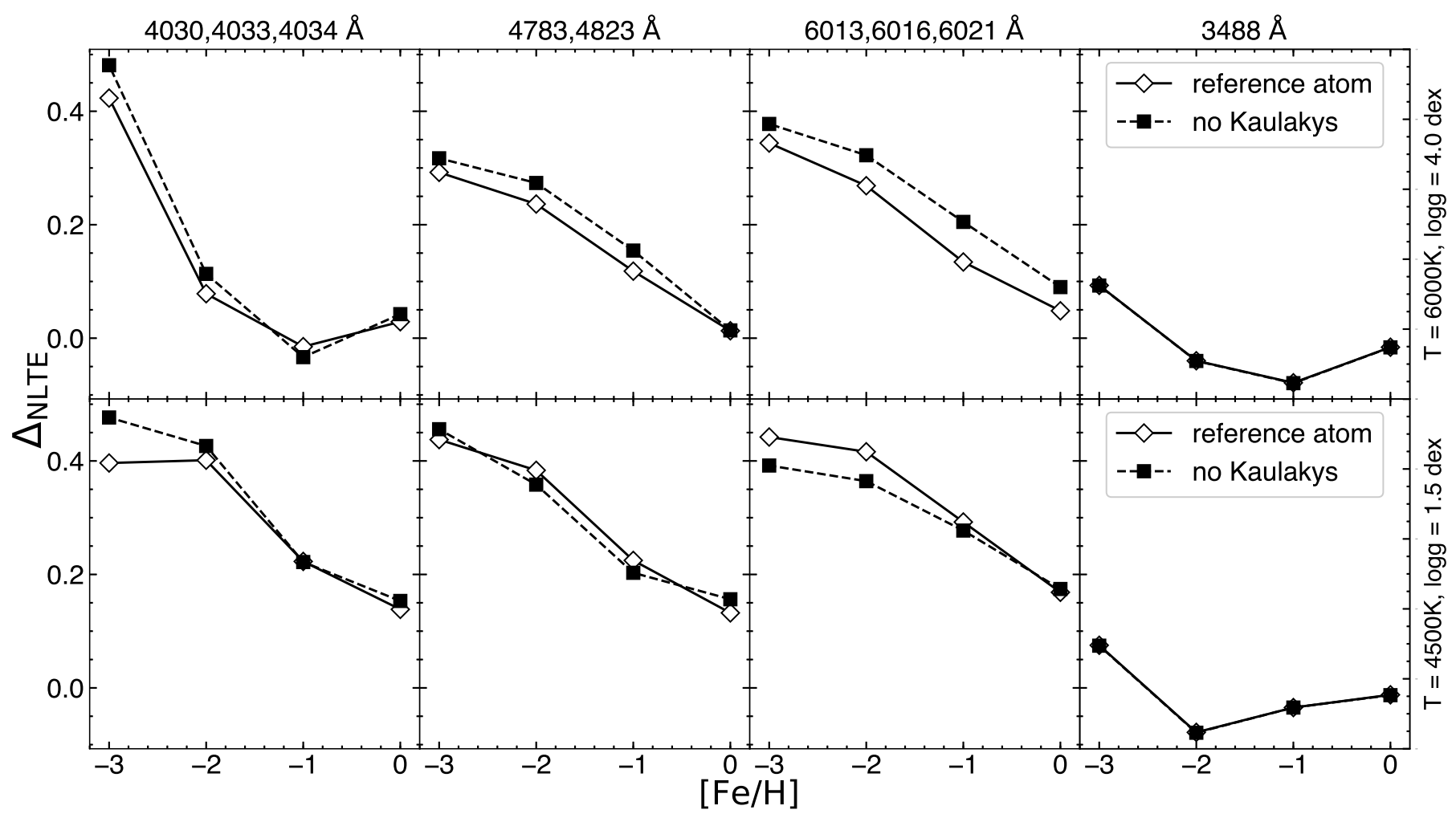

Fig. 10. NLTE abundance corrections for Mn I and Mn II lines. These are computed for a small grid of MARCS model atmospheres representative of dwarfs: $T_{\text {eff }}=6000, \log g=4.0$ (top panels), and red giants: $T_{\text {eff }}=4500, \log g=1.5$ (bottom panels) for a range of metallicities from 0 to -3 dex. Different curves represent the corrections derived using two model atoms, one with and the other without collisions derived using the Kaulakys recipe.

Below we show that 3D NLTE calculations suggest substantial differential effects between Mn I lines of different excitation potentials. These differential effects help to improve the excitation balance (Sect. 4.2), effectively providing the physical basis for the effect, which is mimicked by using inefficient $\mathrm{H}$ collisions in $1 \mathrm{D}$ models.

\section{2. $3 D N L T E$}

\subsubsection{Photon kinematics}

Figure 11 illustrates spatially-resolved NLTE intensity profiles of two Mn I lines in the solar model at the disc centre. All profiles are normalised to the average continuum intensity for the corresponding spectral line in the snapshot. The lines were chosen such that the effect of the HFS is minimal, in order to isolate the effect of granular motions on the profiles. The profiles are taken for every fourth point along each horizontal coordinate in the simulation domain (i.e. for $8 \times 8=64$ points out of 900) in order to not overload the figure. The bisectors for each line component are shown in the right-hand side panels. In addition, the solid curves indicate the profiles extracted from the granule and inter-granular lane in Fig. 12.

Overall, the behaviour of the lines is very similar to that described earlier by Dravins \& Nordlund (1990a, for example in their Fig. 6 for a Sun-like star $\alpha$ Cen A). The weaker highexcitation Mn I line at $5004 \AA$ shows a strong anti-correlation between the depth of the line core and the line shift (right-hand panel). This is, in fact, the weakest unblended solar Mn I line with the EW of only $13 \mathrm{~mA}$. The line profiles with the strongest blue-shift and the highest intensity contrast form above the granules, where the upwards streaming motions of hotter material are characterised by higher velocities, and higher granular temperatures account for the brighter background continuum. The dominant NLTE effect of over-ionisation leads to brightening in the line core. The lack of any pronounced curvature in the bisectors of the blue-shifted components suggests that there is little vertical variation of velocity field in the upflows.

The Mn I line at $4502 \AA$, which is stronger but has the same lower excitation potential as the $5004 \AA$ line, also shows a very broad distribution of line shifts. This line is close to saturation, as is evidenced by the broad, rectangular inner core in the bluest components. Similar to the 5004 line, the bisectors of the blueshifted components, which form above granules, are typically $l$-shaped, that is, the line profiles are nearly symmetric. Although the blue-shifted components are very strong, and are characterised by very (vertically) extended line-formation regions, this again suggests that the vertical variation of velocity fields in the granules is small. On the other hand, the bisectors of the profiles that form above the inter-granular lanes tend to approximate a $c$-shape. These line components are highly asymmetric, their cores are very broad, and tend be skewed to the red. Dravins \& Nordlund (1990a) found that this is a characteristic feature of the lines that form across inter-granular regions with a larger vertical-velocity gradient with depth.

\subsubsection{D test cases}

Calculations of 3D NLTE radiative transfer are very computationally expensive. Hence, we explored whether a simplified 

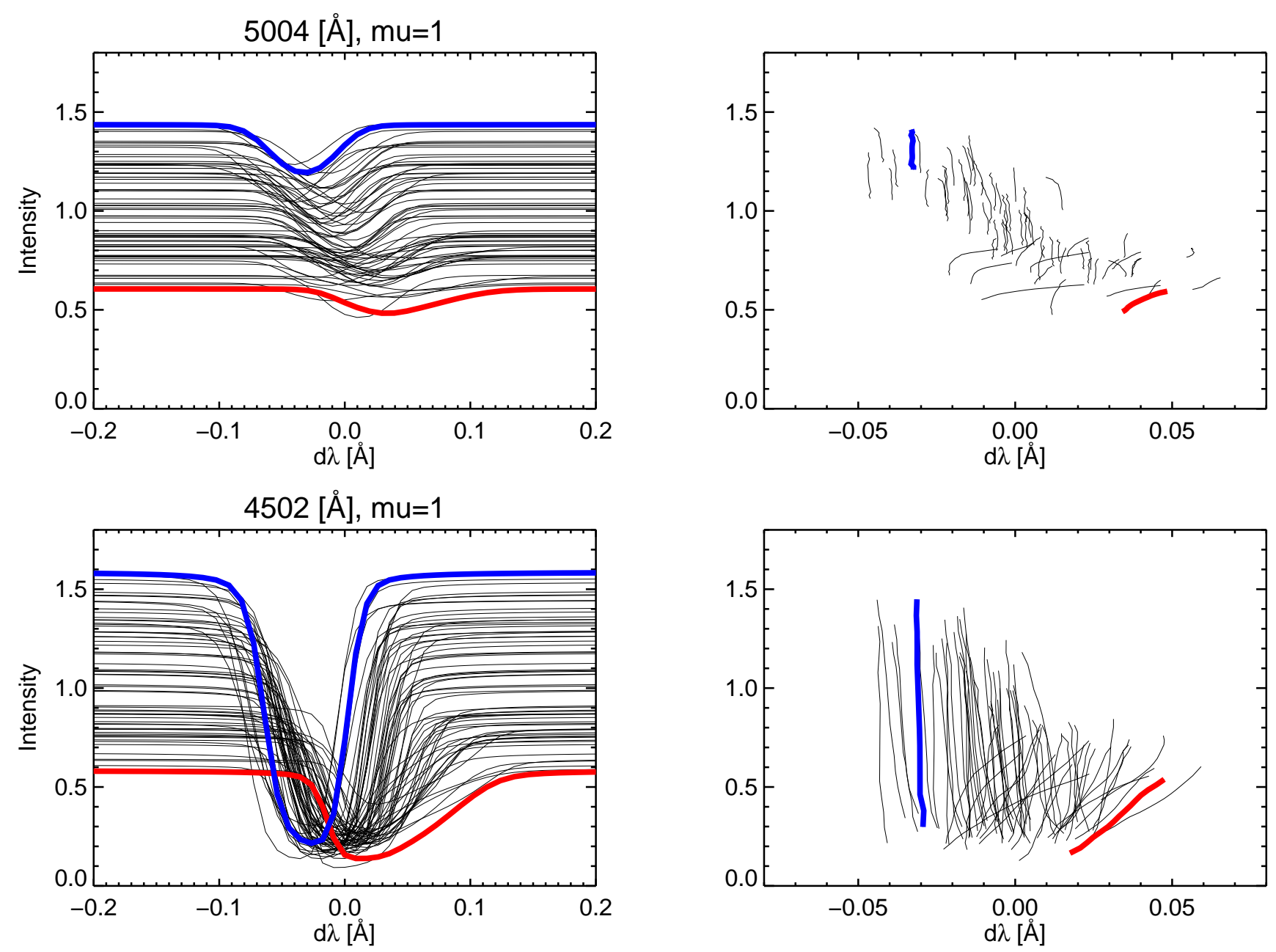

Fig. 11. Spatially resolved line profiles (left panels) and their bisectors (right panels) for solar disc centre in model snapshot 020 . The solid blue curve indicates the line profile extracted from the granule, whereas the red curves correspond to the inter-granular lane. The corresponding surface areas are marked with yellow and blue boxes in Fig. 12.

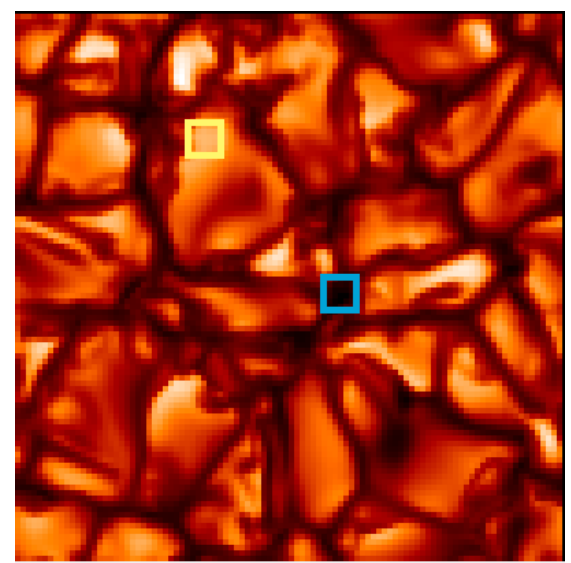

Fig. 12. Monochromatic image of solar granulation at wavelength of $5394 \AA$ at solar disc centre.

treatment of NLTE radiative transfer with 3D simulations offers a suitable alternative to full 3D NLTE calculations.

In particular, we illustrate and discuss the results of calculations obtained using more compact model atmosphere cubes and different radiative transfer solvers (1.5D versus full $3 \mathrm{D})$. We also compare the flux profiles obtained using different solar snapshots. In what follows, we limit the discussion to two Mn I lines only. One of them is a strong resonance line at $5394 \AA$, where the effects of NLTE and 3D convection are most pronounced. The second line is that at $4502 \AA$, discussed in the previous section. This line is least affected by the HFS, hence its shape is a good test case to explore the effects of NLTE and 3D convective flows.

The top left panel of Fig. 13 shows the line profiles computed in full 3D NLTE versus 1.5D calculations (Sect. 3.3). The difference is negligible and it amounts to less than $0.5 \%$ in the flux level, or $<0.01 \mathrm{dex}$ in abundance, that is, far less than the uncertainty incurred by the other sources of error. In the case of metal-poor stars, the differences are also modest and do not exceed a few percent in EW. Since the computational time of 3D NLTE scales as $O\left(n^{4 / 3}\right)$, where $\mathrm{n}$ is the number of grid points (meaning the number of iterations is proportional to the number of grid points in the $z$ direction), it is a reasonable approximation to use 1.5D NLTE calculations, at least for the stars with parameters similar to the models tested in this work.

The top right panel of Fig. 13 illustrates the impact of the model atmosphere resolution. We employ three models: one resampled to the resolution of $5 \times 5 \times 102$, the other with the resolution of $30 \times 30 \times 230$ (our default resolution), as well as the maximum resolution of $100 \times 100 \times 230$ that we can afford 

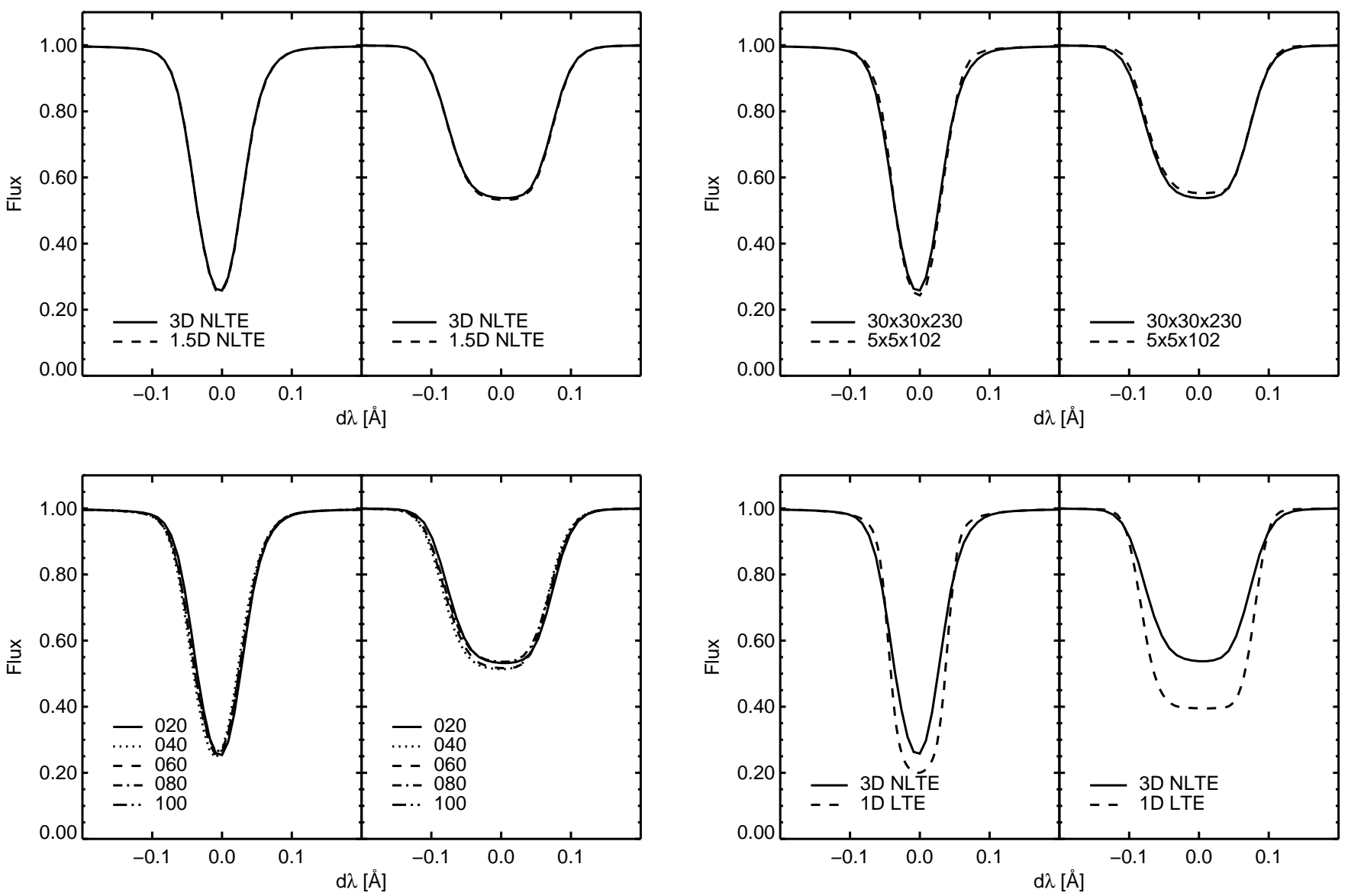

Fig. 13. Comparison of theoretical profiles for Mn I lines at 4502 and $5394 \AA$ for solar snapshot 020. Top left panel: 1.5D NLTE versus full 3D NLTE. Top right panel: line profiles computed with the 3D data cubes scaled down to the resolution of $(x, y, z)=30,30,230$ (solid line) and 5, 5, 102 (dashed line). Bottom left panel: full 3D NLTE for the five solar snapshots. Bottom right panel: 3D NLTE profiles versus 1D LTE profiles.

with the computing time available to us. The comparison of the medium- and maximum- resolution simulation can be found in the appendix. The effects are visible in the line profiles, but they only slightly change the shape of the line and have a very modest effect on the line equivalent width. This effect comes mostly through the impact of velocities on the line opacity, $\kappa(x, v \times(1-v(x) / c))$ as is best seen in lines that are not very sensitive to HFS, such as the $4502 \AA$ line (left panel in Fig. 13). Their shapes are closely approximated by a Gaussian fit, and the effects of velocity fields are easily distinguished. In particular, the red wing of the line appears to be slightly darker in the model with higher resolution. There is also an implicit effect of velocities on the line source function, as it depends on the radiation field at the line frequencies, which is non-local and connected to the velocity distribution at all spatial points along the beam.

We also find (Fig. 13, bottom left panel) that the flux profiles computed using different (regularly-spaced in time) snapshots do not show significant variations. The $5394 \AA$ line changes its depth by $\sim 3 \%$. The position of the line centre changes by a few $\mathrm{m} \AA$. The $4502 \AA$ line is almost insensitive to the temporal effects. This suggests that the statistical properties of velocity fields, temperature, and pressure inhomogeneities in each snapshot are representative of some average distribution values for the relaxed simulation given the values of $T_{\mathrm{eff}}, \log g$, and $[\mathrm{Fe} / \mathrm{H}]$. The temporal variability of spatially resolved profiles has been examined in detail in Dravins (1990); Dravins \& Nordlund (1990b, their Sect. 5). They found that the characteristic evolution of line shapes and depths lacks a unique timescale, that was a manifestation of the lack of a unique scale in the spatial power spectra. Hence, the ensemble averages of spatially resolved line profiles are very similar in different snapshots, as we will also demonstrate in Sect. 4.4 for other models.

Finally, the bottom right panel of Fig. 13 illustrates the line profiles computed with 1D LTE and 3D NLTE. This is a major difference that amounts up to 0.2 dex in the abundance (see next section). Similar to the earlier studies for ions with similarly complex electronic structure, and ionisation potential (e.g. Amarsi et al. 2016; Lind et al. 2017, for Fe I), we find that the effects of 3D NLTE are to weaken the line compared to 1D LTE. Mn I is a photo-ionisation-dominated ion, that is the NLTE effects are driven by a strong non-local radiation field across multiple ionisation channels of the ion in the UV and in the optical. Of all Mn I lines, the resonance lines at 5394 and $5432 \AA$ are most sensitive to this effect, as the lines are comparatively weak in the solar spectrum, and their NLTE effect is mainly caused by the change in the line opacity that scales in the first order as the ratio of the NLTE to LTE number densities of the lower energy level of the transition. The higher-excitation lines, such as that at $4502 \AA$ show more modest NLTE effects in the model atmosphere of the Sun, both in 1D and in 3D.

The results of our test calculations indicate that vertical radiative transfer in NLTE with full 3D data cubes at the resolution of $(x, y, z)=30,30,230$ is the most suitable approach and we employed this method in our calculations of Mn abundances for the Sun (Sect. 4.3) and the benchmark stars (Sect. 4.6). The approach allows affordable 3D NLTE calculations within 


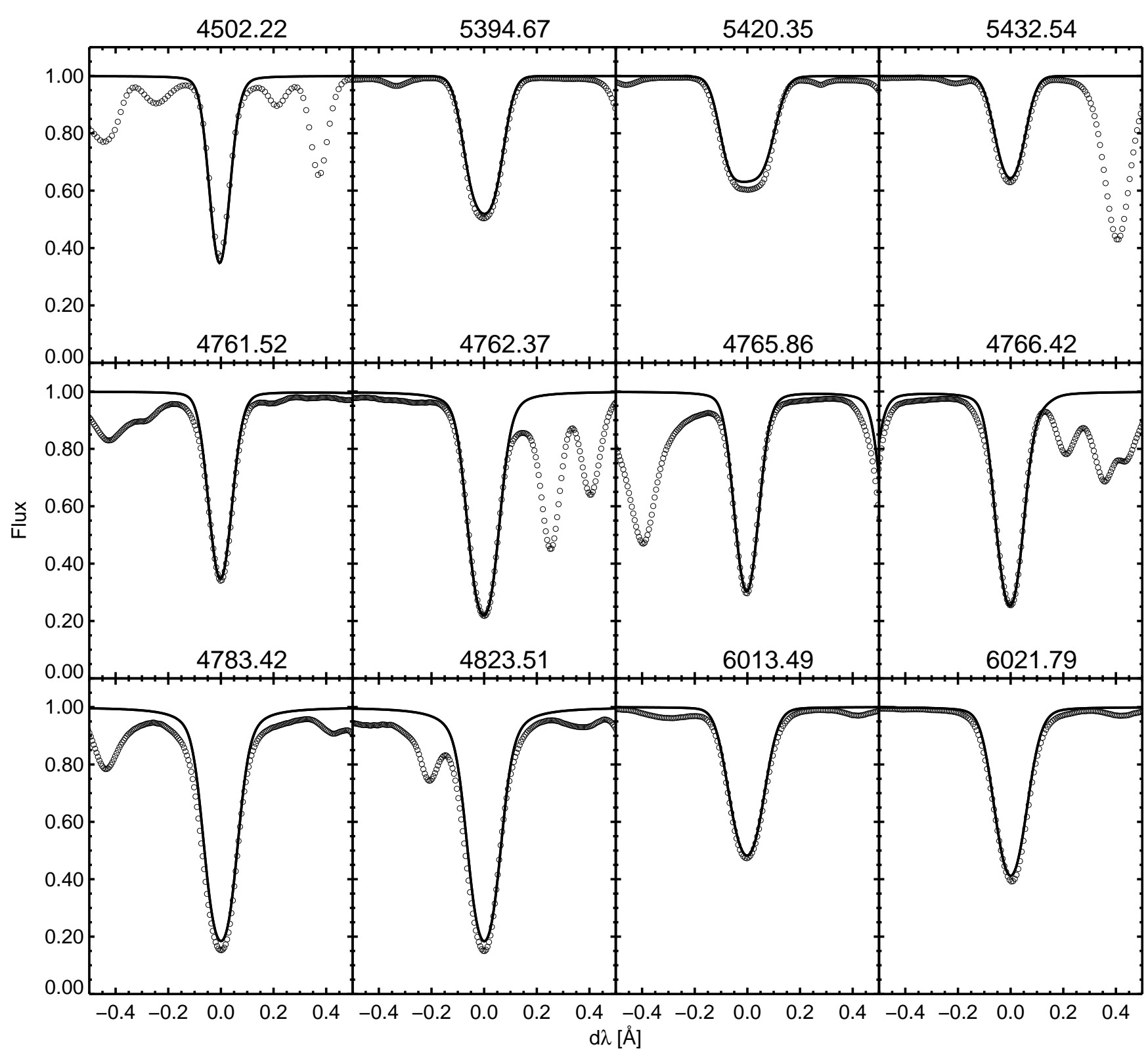

Fig. 14. Comparison of 3D NLTE line profiles for selected Mn I lines with observed solar flux spectrum. All model profiles were generated using the same Mn abundance of $5.47 \mathrm{dex}$.

reasonable timescales and provides line profiles consistent with the original resolution of the $3 \mathrm{D}$ cubes, as well as, with the full $3 \mathrm{D}$ radiative transfer calculations.

\subsection{Solar Mn abundance}

The Sun is a metal-rich star, and as such, the spectral lines in most cases appear to suffer from blending and/or strong sensitivity to damping or turbulence. As a matter of fact, most Mn I lines are not very useful, being either blended or too strong, with a few exceptions. Nonetheless, we start with the analysis of all Mn I lines that are typically used in the analysis of FGK stars, and later assume a more conservative approach that takes into account only the most reliable features.

The LTE analysis of the solar Mn I lines is carried out using the SIU spectrum synthesis code (Reetz 1999). SIU allows interactive spectral fitting, which is very convenient to test for the presence and effects of blends and asymmetries within a line.
This is a very accurate approach, especially when applied to ultra-high-resolution spectra, such as the solar atlases, and has been commonly exploited in the literature.

Figure 14 presents some examples of 3D NLTE line profiles for the solar spectrum. Note that in this plot, we have not adjusted the Mn abundances, but assumed the abundance of $5.47 \mathrm{dex}$, as recommended by earlier studies of the solar Mn abundance. Most of the Mn I lines are very broad, with the width of $0.2-0.3 \AA$, which is a consequence of the HFS. It is obvious that the available atomic and HFS data describe the line profiles remarkably well, both in terms of the asymmetries and the multi-component structure. The latter is particularly prominent in the cores of the weak resonance or ground-state lines, such as the 5394 and $5516 \AA$ line. The core of the line at $5420 \AA$ is slightly too weak, compared with the observations. This could be due to its sensitivity to activity (Wise et al. 2018). Note that also the 5394, 5432, and 6013 lines could be affected (Wise et al. 2018), and in particular, the $5394 \AA$ line has a long history of research owing 

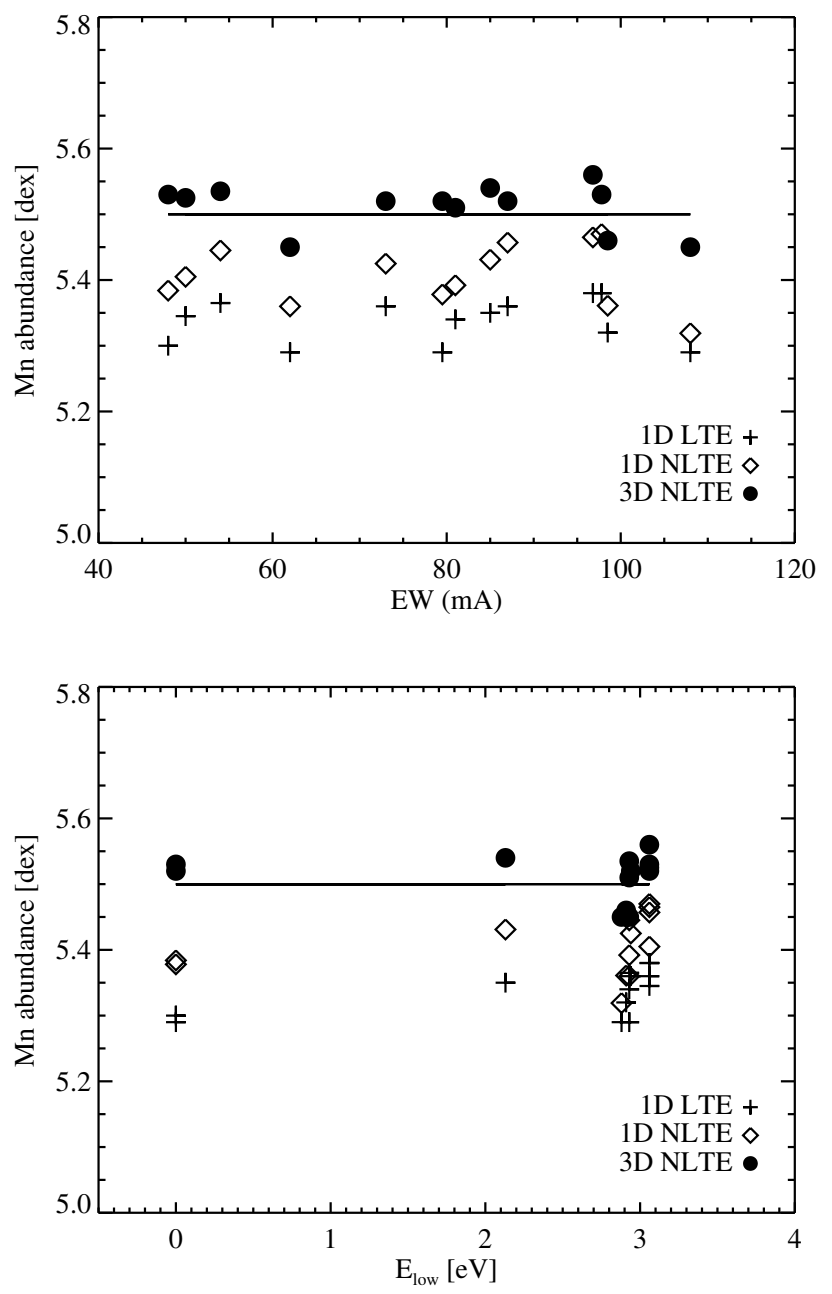

Fig. 15. Mn abundances determined from individual Mn I lines in solar flux spectrum using different models: 1D LTE, 1D NLTE, and 3D NLTE. The solid line denotes the Mn abundances measured in CI chondrites, $5.50 \pm 0.03$ dex (Lodders 2003).

to its variation with the global solar activity cycle (Danilovic \& Vince 2005; Livingston et al. 2007). Some studies suggest that the line is not as sensitive to granular motions as other features, because of its wide HFS, and is more sensitive to the intergranular magnetic concentrations, similar to the $\mathrm{CH} G$-band (Vitas et al. 2009). According to our 3D NLTE results, however, the $5394 \AA$ line is sensitive to convection and shows a significant difference between modelling with 1D hydrostatic and 3D convective models. Overall, the agreement of the model 3D NLTE line profiles with the observed solar data suggests that the atomic data quality and the physical quality of spectral models are sufficient to satisfactorily describe the properties of Mn lines in the solar spectrum.

The abundances determined from the Mn I lines that we view as reliable photospheric abundance diagnostics are shown in Fig. 15. The major challenge in the analysis of the solar spectrum is the contamination of line profiles by blends. These are present in the wings of the strong Mn I lines, such as the 4761, 4762,4765 , and $4766 \AA$, but also the lines of multiplet 18 (4783 and $4823 \AA$ ). Besides, there are no quantum-mechanical data for elastic collisions with $\mathrm{H}$ atoms for the two latter lines (Barklem et al. 2000), hence, we have to resort to the standard Unsöld formalism that carries an additional source of an uncertainty. We disregard such lines when computing the solar abundance of
Mn. The 1D NLTE and 3D NLTE Mn abundances are derived by applying the corrections computed using MULTI2.3 and MULTI3D for the individual spectral lines.

In 1D LTE, the low-excitation potential and/or very strong lines give a lower abundance of Mn compared to the high-EP (weaker) lines. The difference is not large, but significant given the very quality of the observed data and well-constrained fundamental parameters of the Sun. The effects on NLTE in the solar atmosphere are moderate and change the average solar abundance by only $\sim 0.02-0.07$ dex. NLTE alone does not help to improve the solar excitation balance. More important is the combined influence of convection and NLTE. In the case of NLTE calculations with the 3D convective solar model, the effect of over-ionisation is amplified owing to stronger background radiation fields in the granules. The 3D NLTE-1D LTE difference is equivalent to $\sim 0.22$ dex in abundance for the resonance lines, but 0.15 dex for the higher excitation lines of other multiplets.

Our solar abundances of $\mathrm{Mn}$ are $5.34 \pm 0.04 \mathrm{dex}$ in LTE, $5.41 \pm 0.05 \mathrm{dex}$ in NLTE, and $5.52 \pm 0.04$ in 3D NLTE. The 1D NLTE and 3D NLTE values are consistent with the meteoritic abundance of Mn 5.50 \pm 0.03 dex reported by Lodders (2003). Our 1D LTE result is $\sim 0.1$ dex higher compared to the previous estimate reported in Bergemann \& Gehren (2007), $5.23 \pm$ 0.1 dex. On the other hand, it matches very well with our LTE estimate obtained using the MAFAGS solar model, the revised transition probabilities, and the SIU spectrum synthesis code in Blackwell-Whitehead \& Bergemann (2007), $5.33 \pm 0.1$ dex. The new 1D NLTE values are also higher than the estimates in Bergemann \& Gehren (2007). In both cases, 1D LTE and 1D NLTE, the difference is due to the revision of the transition probabilities and the NLTE model atom.

\subsection{D NLTE effects in metal-poor model atmospheres}

Our analysis of line formation with 1D models suggests that the majority of Mn lines are sensitive to NLTE. Also the calculations for the 3D hydrodynamical model of the Sun indicate a strong impact of convection on the line profiles, which does not cancel the NLTE effects, but rather amplifies them for the most spectral features of Mn I.

In this section, we present the analysis of line formation in several model atmospheres of metal-poor stars. We explore the models with parameters with $T_{\text {eff }}=6500 \mathrm{~K}, \log g=4$ dex, $[\mathrm{Fe} / \mathrm{H}]=-1$ and $T_{\text {eff }}=4500 \mathrm{~K}, \log g=2,[\mathrm{Fe} / \mathrm{H}]=-1$, as well as their metal-poor counterparts with $[\mathrm{Fe} / \mathrm{H}]=-2$. For simplicity and didactic clarity, all calculations were performed assuming a scaled solar Mn abundance. Deviations from this assumption may affect the line formation, yet at this stage we are interested in the combined effects of 3D radiative transfer in convective models and NLTE and the impact on Mn I lines with different properties. We explore which lines are least sensitive to these effects, and employ them in the abundance analysis in the follow-up study of a larger stellar sample.

Our results for the four 3D models are illustrated in Fig. 16. We also overplot the predictions of 1D LTE modelling computed using the same Mn model atom and the same Mn abundance. Similar to the solar model, the 3D NLTE line profiles are much weaker than the 1D LTE counterparts. This is the consequence of over-ionisation, which is stronger in metal-poor conditions due to reduced line blanketing and stronger UV radiation fluxes. The topology of convection in FGK stars is set by mass conservation. The hotter rising granules occupy the dominant $(\sim 2 / 3)$ part of the stellar surface, whereas the cooler downdrafts are confined to narrow inter-granular lanes (Stein \& Nordlund 1989; 

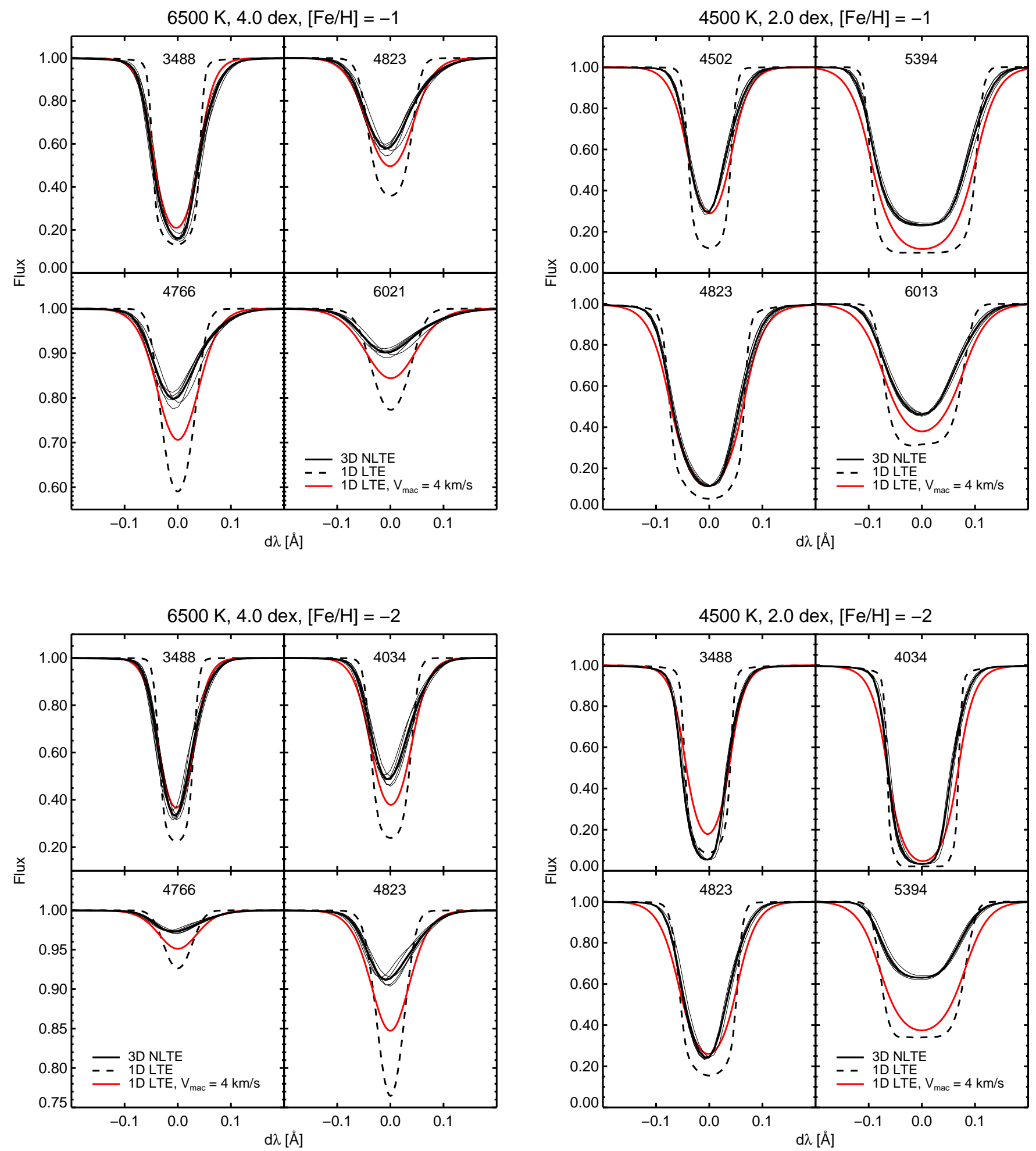

Fig. 16. Line profiles of selected $\mathrm{Mn} \mathrm{I}$ lines in dwarf and RGB models with metallicities $[\mathrm{Fe} / \mathrm{H}]=-1$ (top panels) and $[\mathrm{Fe} / \mathrm{H}]=-2($ bottom panels $)$. Thick solid curves correspond to the averaged profiles from five snapshots taken at equidistant time steps. Thin lines are the profiles from individual snapshots. 1D LTE profiles are computed assuming $\xi_{\mathrm{t}}=1 \mathrm{~km} \mathrm{~s}^{-1}$. The 1D LTE profiles would be stronger, if larger $\xi_{\mathrm{t}}$ values were assumed. Note that different spectral lines of Mn I and Mn II are shown in the panels.

Nordlund et al. 2009), although the area subtended by the down-flows increases with depth. Hence, over-ionisation in the granules is the dominant NLTE effect that defines the appearance of the spatially integrated line profiles. In contrast to the Sun, where the effects of convective shifts were marginal, the integrated flux profiles in the metal-poor models show strong asymmetries, with typical blue-shifted cores and skewed wings. This is best seen by comparing the symmetric 1D profiles, convolved with an arbitrary macro-turbulence and the 3D profiles, which do not assume any ad-hoc broadening. Also the line profiles from individual snapshots are remarkably similar to their temporarily averaged counterparts, although a slightly more 
pronounced temporal variation is seen in the NLTE line profiles computed using the 3D models of dwarfs (as in Fig. 16).

The differences between 1D LTE and 3D NLTE profiles are very large, with only a few notable exceptions. These exceptions are the blue lines of Mn II, such as the 3488 and $3497 \AA$ lines. For dwarfs, these low-excitation Mn II features are nearly invariant to the changes in the model atmosphere structure, and they indeed show very similar line shapes and equivalent widths, regardless of whether one adopts LTE or NLTE radiative transfer. Also a standard choice of the micro-turbulence $\xi_{\mathrm{t}}=1 \mathrm{~km} \mathrm{~s}^{-1}$ appears to be satisfactory for a dwarf model, producing a line profile, which closely resembles the full 3D NLTE profile of the Mn II $3488 \AA$ line even in the model with $[\mathrm{Fe} / \mathrm{H}]=-2$. For the computational complexity of 3D NLTE modelling, we are unable to explore a larger parameter space of $3 \mathrm{D}$ models in this work, but the behaviour of the models with metallicity suggests that this conclusion may hold also for other stars on the mainsequence, as long as $[\mathrm{Fe} / \mathrm{H}]$ is not in the extremely metal-poor domain.

This fortunate result does not hold for the atmospheres of giants, however, and neither does it generally hold for the Mn I lines. The $3488 \AA$ line of Mn II suffers from non-negligible deviations from NLTE in the RGB models, being significantly stronger in 3D NLTE. On the other hand, the 3D NLTE abundances of all lines of Mn I are significantly higher compared to 1D LTE. This is best illustrated by exploring the differences between 1D LTE and 3D NLTE, and 1D NLTE and 3D NLTE abundances derived from the line of a given equivalent width. This quantity is typically referred to as a 3D NLTE abundance correction (3D NLTE - 1D LTE).

The top panel of Fig. 17 shows that the 3D NLTE abundance corrections amount to 0.25 dex for most Mn I lines in the atmospheres of dwarfs, but in the models of RGB stars the (3D NLTE - 1D LTE) abundance differences tend to be significantly larger. In all cases, 1D analysis tends to substantially underestimate the $\mathrm{Mn}$ abundance. In particular, for the lines of multiples 18 and 32 (e.g. 4823 and $6016 \AA$ ) we find the abundance corrections of $0.3-0.4$ dex in metal-poor models, whereas the blue resonance lines at 4030-4034 $\AA$ show even larger $3 \mathrm{D}$ NLTE corrections of $\sim 0.5 \mathrm{dex}$ at $[\mathrm{Fe} / \mathrm{H}]=-2$. This is a very interesting result. On the one hand, our results suggest that the large abundance discrepancies and excitation imbalance seen in the analysis of RGB stars (Bonifacio et al. 2009) may indeed be accounted for by 3D NLTE. On the other hand, we also find that 3D NLTE effects do not significantly impact the excitation balance in the atmospheres of very metal-poor dwarfs, as 3D NLTE corrections are similar for the lines of all Mn I multiplets. This is why this effect may go undetected in 1D LTE studies of stars with similar parameters (e.g. the study of HD 84937 by Sneden et al. 2016, or Mishenina et al. 2015). Also the 3D NLTE corrections for the Mn II lines are very small in the metal-poor atmospheres of dwarfs, and do not exceed -0.05 dex at $[\mathrm{Fe} / \mathrm{H}]=-2$.

Figure 17 also suggests that, whereas 1D LTE always underestimates abundances for the Mn I lines, there are a few Mn I lines that can be reliably modelled with 1D NLTE. These are the lines of multiplets 9 (e.g. 4055, 4070, and $4082 \AA$ ), 23 (e.g. 4761, 4762, 4765, and $4766 \AA$ ), 24 (e.g. 4436, 4451, 4498, and $4502 \AA$ ), and 32 (e.g. 6013, 6016, and $6021 \AA$ ). For these features, the differences between 1D NLTE and 3D NLTE are marginal, and do not exceed 0.1 dex in the lowest metallicity $([\mathrm{Fe} / \mathrm{H}]=-2)$ models of both dwarf and RGB stars. The lines of other multiplets show a very significant systematic deviation from 1D NLTE, caused by the impact of convective
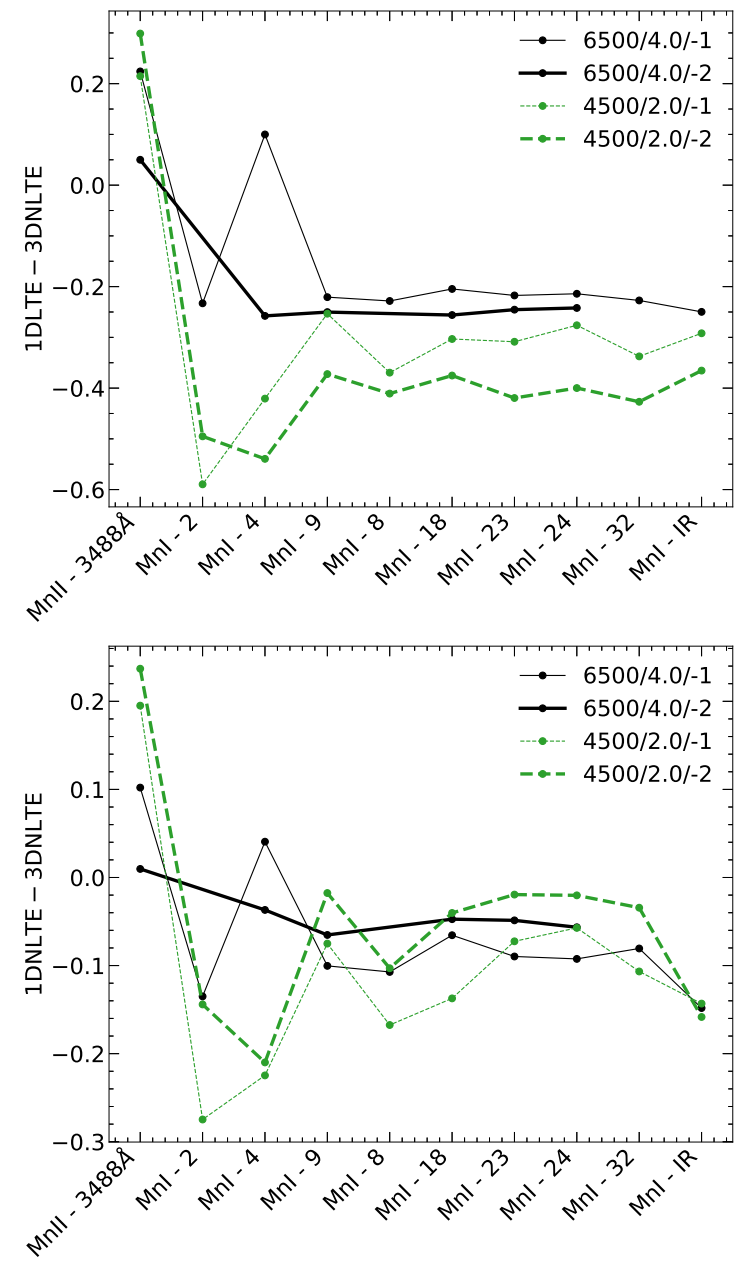

Fig. 17. 3D NLTE abundance corrections for selected models.

inhomogeneities. Additionally, for dwarfs, the impact of convection is modest and indeed, most optical Mn I lines, including the resonance $\mathrm{Mn}$ I triplet at $403 \mathrm{~nm}$, would be suitable for abundance diagnostic, leading to a modest bias of $\sim_{-0.15}^{+0.05}$ dex. The exact value would also depend on the abundance of Mn itself, and additionally on the assumed value of micro-turbulence in the 1D models. So our estimates of 3D NLTE abundance corrections may not be directly applicable to the 1D LTE measurements in the observed stars. Rather we recommend employing those lines, which according to our tests, show minimal impact of convection and/or NLTE.

The IR lines of MnI, like those that are used in the H-band abundance analysis (Shetrone et al. 2015), show significant departures from 1D and LTE. However, it is remarkable that all three Mn lines that we tested in this work show very similar 3D NLTE - 1D NLTE differences in dwarf and RGB models. In effect, the abundances for all these models are systematically underestimated by -0.15 dex with respect to $1 \mathrm{D}$ NLTE, and the bias appears to be insensitive to metallicity of the model. It appears reasonable then to apply a positive correction to all H-band Mn 1D NLTE abundance measurements to obtain physically unbiased results.

\section{5. $3 D$ LTE versus $3 D$ NLTE}

Figure 18 compares the line profiles computed using 3D LTE, 3D NLTE, and 1D LTE. The latter is convolved with a macroturbulence of $4 \mathrm{~km} \mathrm{~s}^{-1}$. 
$6500 \mathrm{~K}, 4.0 \mathrm{dex},[\mathrm{Fe} / \mathrm{H}]=-1$

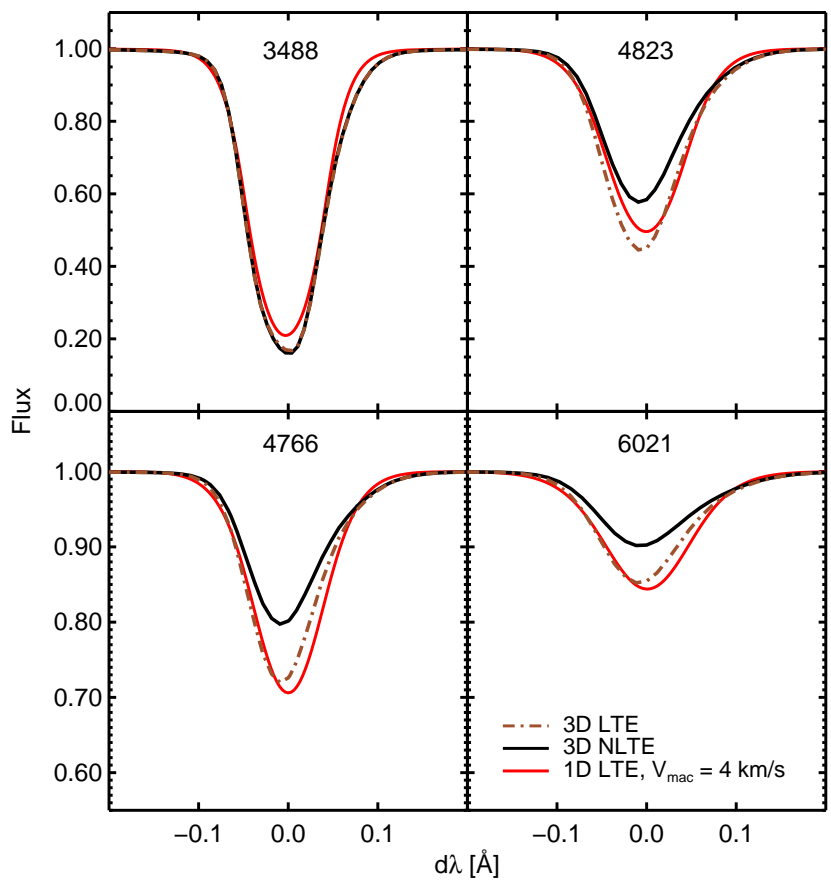

$6500 \mathrm{~K}, 4.0 \mathrm{dex},[\mathrm{Fe} / \mathrm{H}]=-2$

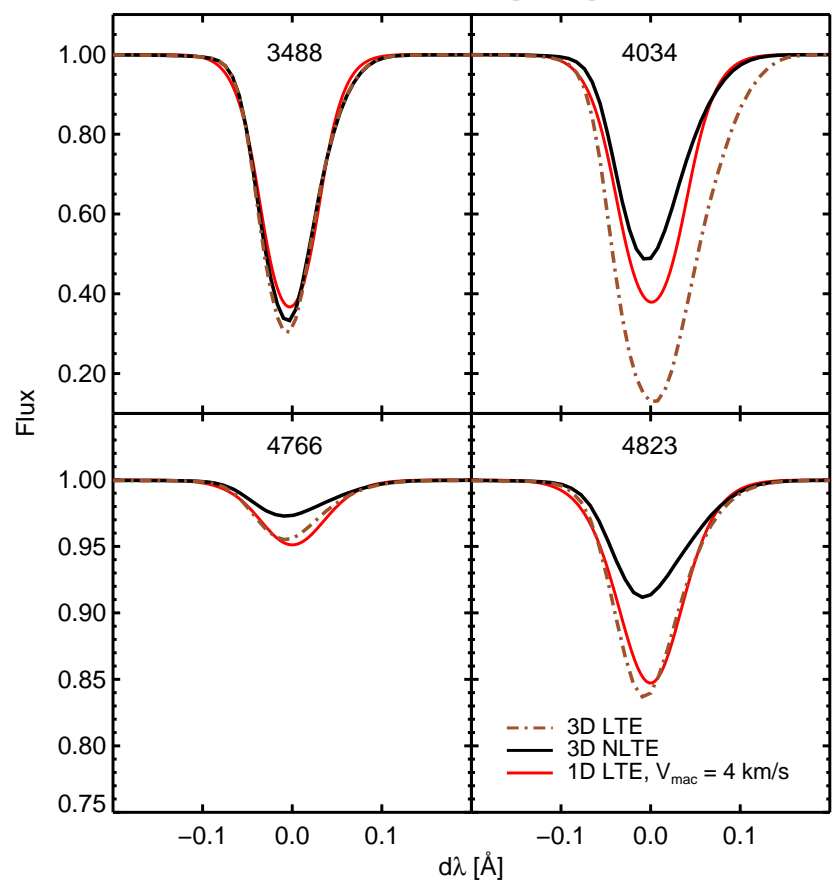

$4500 \mathrm{~K}, 2.0 \mathrm{dex},[\mathrm{Fe} / \mathrm{H}]=-1$

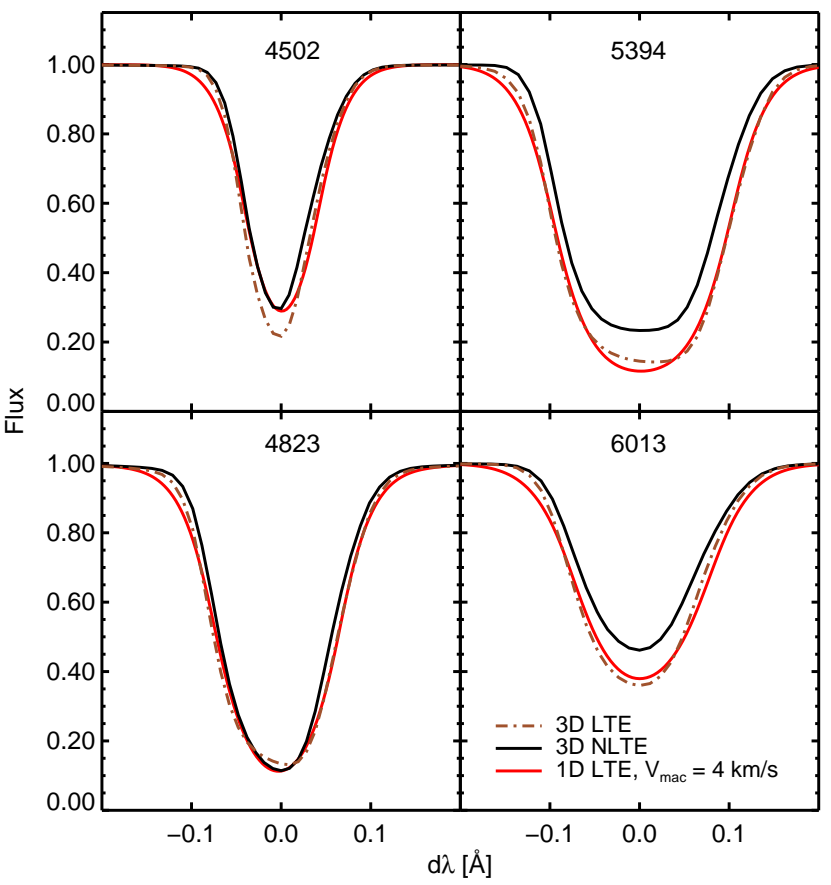

$4500 \mathrm{~K}, 2.0 \mathrm{dex},[\mathrm{Fe} / \mathrm{H}]=-2$

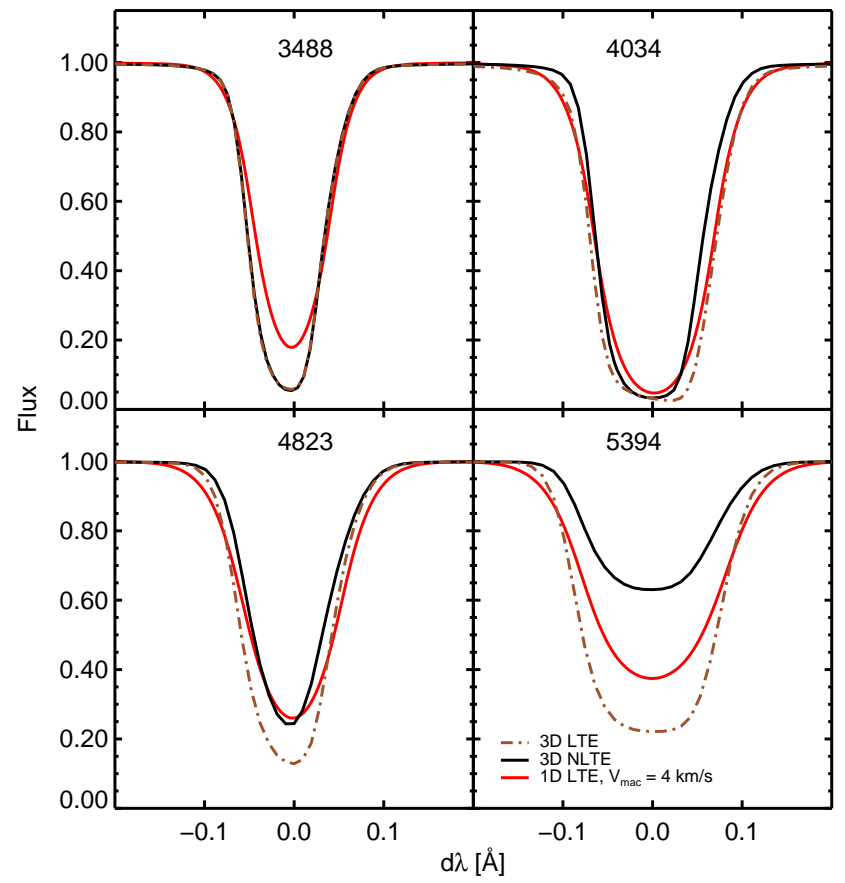

Fig. 18. Same as Fig. 16, but thick dotted-dashed brown curves now denote the profiles from 3D LTE calculations for the same five snapshots.

For the $[\mathrm{Fe} / \mathrm{H}]=-1 \mathrm{~d}$ warf model, the $3 \mathrm{D}$ LTE line profile of the $\mathrm{Mn}$ II line at $3488 \AA$ A closely resembles the equivalent 1D LTE profile. The Mn I lines at 4766 and $6021 \AA$ are slightly shallower in 3D LTE compared to 1D LTE, but deeper for the $4823 \AA$ line. On the other hand, all lines of Mn I are much fainter in 3D NLTE compared to 3D LTE, suggesting that a 3D LTE analysis would strongly underestimate the $\mathrm{Mn}$ abundances.

This tendency is qualitatively very similar in the dwarf model at $[\mathrm{Fe} / \mathrm{H}]=-2$. One notable exception is that the resonance line at $4034 \AA$ is now exceptionally strong in 3D LTE, much stronger than the profiles computed using 1D LTE and 3D NLTE. This behaviour is also known for strong resonance lines of other chemical species. The major implication of this is that 3D LTE modelling is unlikely to solve the excitation imbalance in Mn, which is well-known from 1D LTE studies (e.g. Bonifacio et al. 2009; Sneden et al. 2016). The unfortunate problem is that even in 1D LTE the resonance Mn I lines yield significantly lower abundances, relative to their higher excitation counterparts. The 3D LTE line profiles of the resonance lines are stronger than their 1D LTE counterparts, computed assuming the same 
Mn abundance, meaning that the 3D LTE abundances of these lines must be reduced to recover the correct equivalent width. For the higher-excitation lines, the differences between 1D and $3 \mathrm{D} \mathrm{LTE}$ at $[\mathrm{Fe} / \mathrm{H}]=-2$ are not large, because the lines are very weak, and form deep in the atmosphere, at $\log \tau_{5000}>-2$, where the temperature inhomogeneities are not pronounced and the average structure of the $1 \mathrm{D}$ hydrostatic models closely resembles that of 3D models (see, e.g. Fig. 6).

The behaviour is very similar in the $3 \mathrm{D}$ models of giants. 3D LTE line profiles are not too different from 1D LTE at $[\mathrm{Fe} / \mathrm{H}]=-1$ despite the modest effect of convection on the line shapes, but they are always stronger compared to 3D NLTE. The differences are exacerbated at low metallicity, $[\mathrm{Fe} / \mathrm{H}]=-2$, where 3D LTE calculations greatly overestimate the strength of all Mn I lines compared to 3D NLTE. The UV Mn II line at $3488 \AA$ shows very small NLTE effects in the metal-poor RGB model, in fact, its 3D LTE and 3D NLTE profiles are very similar. On the other hand, the 1D LTE assumption underestimates the strength of the Mn II line, thus overestimating the Mn abundance derived from this feature.

\subsection{Benchmark metal-poor stars}

The Mn abundances in the stellar spectra were computed by matching the observed equivalent widths to the grids of 1D LTE and 1D NLTE spectral lines computed using MULTI2.3. The equivalent widths were measured from the UVES-POP spectra of these stars using the SIU code. Unlike in our analysis of the Sun, we have chosen to not employ SIU for abundance measurements in metal-poor stars, as blending is not a problem anymore and a detailed spectrum synthesis is unnecessary. Also MULTI2.3 has an advantage in that it includes background scattering, which is essential for the blue and UV lines. 3D NLTE corrections were computed separately and applied to the 1D LTE abundances derived using the measured EWs.

The results for all three benchmark metal-poor stars are shown in Fig. 19. The error bars correspond to the uncertainties of the EW measurements. We show the abundances derived using the MAFAGS-OS models (left panels) and the MARCS models (right panels) in order to illustrate the impact of the 1D model atmospheres. Overall, the results obtained MAFAGS-OS and MARCS models are in agreement, especially for the model of the metal-poor RGB star HD 122563. The differences can generally be explained by the small differences in the input parameters of the models. In particular, the MAFAGS-OS models assume the following parameters: (a) HD 122563: $T_{\text {eff }}=4600 \mathrm{~K}, \log g=1.60 \mathrm{dex},[\mathrm{Fe} / \mathrm{H}]=$ $-2.5 \mathrm{dex}$; (b) HD 140283: $T_{\text {eff }}=5773 \mathrm{~K}, \log g=3.66 \mathrm{dex},[\mathrm{Fe} / \mathrm{H}]$ $=-2.38$ dex; and (c) HD 84937: $T_{\text {eff }}=6350 \mathrm{~K}, \log g=4.09$ dex, $[\mathrm{Fe} / \mathrm{H}]=-2.15 \mathrm{dex}$. This is most likely at the origin of the somewhat lower abundances that we get for HD 84937. It is also noteworthy that MARCS models lead to slightly more consistent abundances from Mn I lines for all three reference stars.

Regardless of the choice of 1D models, 1D LTE modelling reveals a significant ionisation imbalance, in that the Mn I lines give systematically lower abundances compared to Mn II lines, confirming the results reported in the literature (Johnson 2002). The difference is most pronounced for the RGB star HD 122563, for which the offset is close to $0.7 \mathrm{dex}$. We also confirm the Mn I excitation imbalance in all three stars, with strong resonance lines of Mn I producing systematically lower abundances compared to higher excitation lines. For HD 122563, the offset between the lines of multiplet 4 (the 4030-4034 $\AA$ triplet) and the lines of other multiplets is $\sim 0.2$ dex, whereas for the subgiant HD 140283 and turn-off star HD 84937 the offset is slightly larger, of the order $\sim 0.3$ dex.

The ionisation balance is significantly improved for all three stars in 1D NLTE. In particular, for HD 84937 and HD 140283, the abundances derived from the high-excitation lines are now consistent with the abundances derived from the Mn II lines. For HD 122563, the ionisation balance in 1D NLTE is only partially improved, but there is still a differential effect of -0.2 dex for the high-excitation lines and -0.5 dex for the low-excitation lines.

The improvement is most striking in 3D NLTE. The 3D NLTE abundance corrections are very large and positive for both ionisation stages. For HD 122563, this brings Mn I and Mn II lines into agreement. For HD 84937 and HD 140283, the 3D NLTE abundances are higher compared to 1D NLTE, however, the differential effect is not as large. It should be emphasised that stellar parameters of these three stars are wellconstrained by independent methods, and the fact that ionisation balance is satisfied in 3D NLTE, without resorting to ad-hoc parameters, like micro-turbulence in $1 \mathrm{D}$, is remarkable. For comparison, to explain the ionisation imbalance in HD 122563 by the error in stellar parameters, its $T_{\text {eff }}$ would have to be increased to $4900 \mathrm{~K}$ (that would bring the resonance lines up by approximately +0.7 dex to be consistent with Mn II), or alternatively the $\xi_{\mathrm{t}}$ increased to $3.5 \mathrm{~km} \mathrm{~s}^{-1}$ dex that would push the Mn II abundances down by approximately -0.7 dex. Decreasing the $\log g$ might help to increase the abundance from the resonance lines, but a change to $\log g<0.5$ would be necessary. All these changes in stellar parameters can be ruled out, given the robust constraints from asteroseismology and interferometry (Creevey et al. 2012; Karovicova et al. 2018; Creevey et al. 2019). Also micro-turbulence, despite being a free parameter, is relatively well-constrained in the literature (Bergemann et al. 2012; Afşar et al. 2016).

Sneden et al. (2016) recently analysed HD 84937 by employing 1D LTE models. They adopted somewhat different stellar parameters for this metal-poor turn-off star: $T_{\text {eff }}=6300 \mathrm{~K}$, $\log g=4.0,[\mathrm{Fe} / \mathrm{H}]=-2.15$, and $\xi_{\mathrm{t}}=1.5 \mathrm{~km} \mathrm{~s}^{-1}$. Their estimates of $[\mathrm{Mn} / \mathrm{Fe}]$ for this star are approximately -0.25 for the Mn II lines and approximately -0.3 for the Mn I lines (their Fig. 7). On the other hand, they also tabulate the abundances derived by taking the ratios of the elements derived from the lines in the same ionisation stage (e.g. Mn I to Fe I and Mn II to Fe II). In the latter case, their values are -0.42 for the Mn II lines and -0.46 for the Mn I lines (their Table 5).

Our 1D LTE estimates for HD 84937 are $[\mathrm{Mn} / \mathrm{Fe}]=$ -0.24 dex, as derived from the Mn II lines, and $[\mathrm{Mn} / \mathrm{Fe}]=$ -0.47 dex for the high-excitation Mn I lines. Similar to Sneden et al. (2016), we find that the MnI triplet lines give the abundances that are $\sim 0.2$ dex lower compared to the highexcitation lines. Our abundances derived from the Mn I lines may appear to be lower, but this is likely the consequence of the adopted metallicity. Indeed, we adopt $[\mathrm{Fe} / \mathrm{H}]=-2.0 \mathrm{dex}$ in this work. This is consistent with our $\langle 3 \mathrm{D}\rangle$ NLTE estimate in Bergemann et al. (2012), where we found metallicities in the range from $[\mathrm{Fe} / \mathrm{H}]=-2.04$ to $-1.95 \mathrm{dex}$, depending on the choice of the H-scaling factor. Amarsi et al. (2016) find $[\mathrm{Fe} / \mathrm{H}]=-1.96 \pm 0.02$ (stat) \pm 0.04 (sys) dex from the full $3 \mathrm{D}$ NLTE Fe analysis. On the other hand, Sneden et al. (2016) adopt a very low metallicity, $[\mathrm{Fe} / \mathrm{H}]=-2.15 \mathrm{dex}$, that leads to significantly higher $\mathrm{Mn}$ abundances. It is not clear which of the estimates from Sneden et al. (2016) are to be given preference and which of their two methodological approaches is more consistent with our estimate. Contrary to that study, we do 

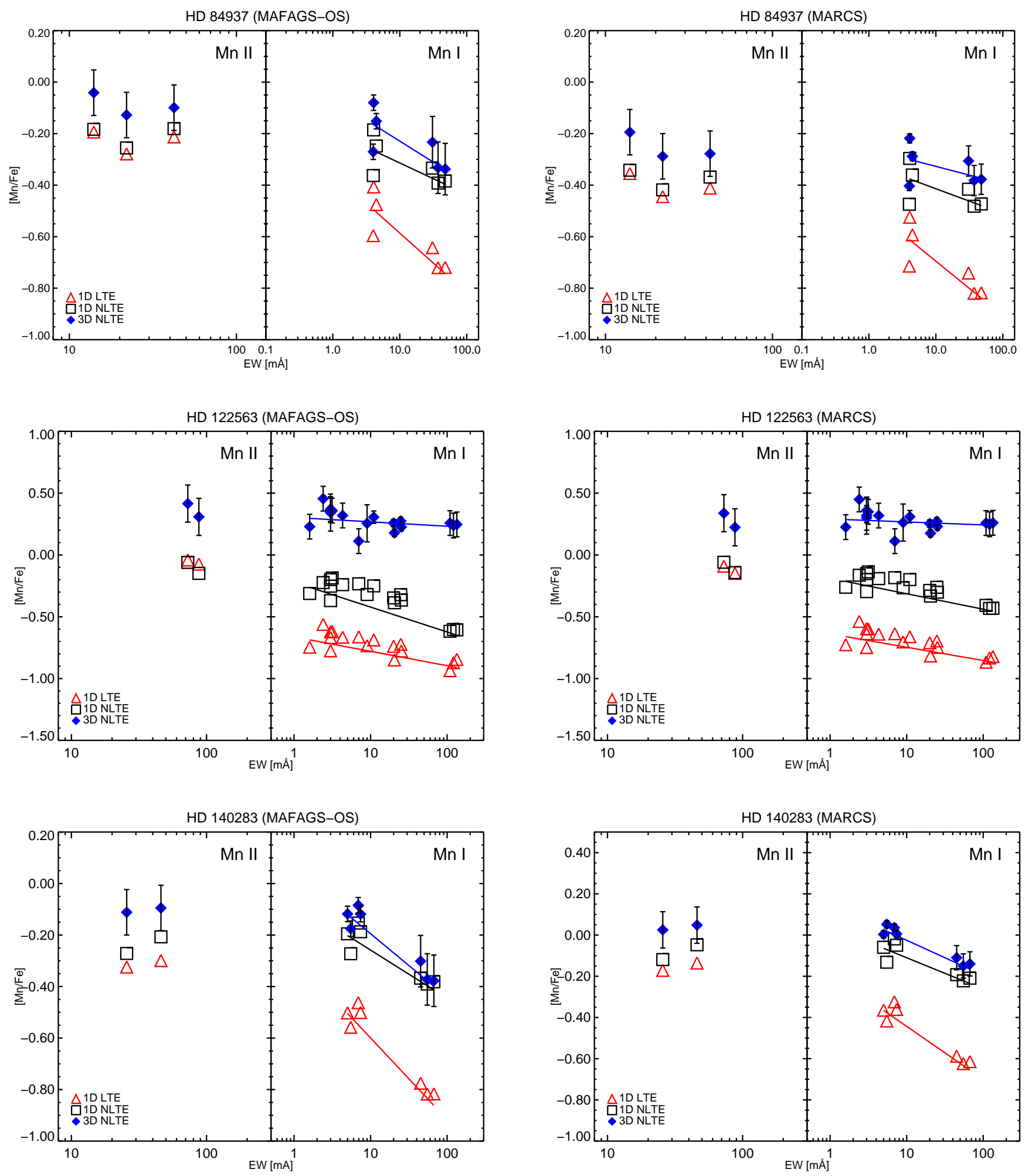

Fig. 19. Mn abundances in three metal-poor stars. Left panels: the abundances determined using MAFAGS-OS and 3D models. Right panels: the abundances determined using the MARCS and 3D models.

not consider lines with EWs $\lesssim 3 \mathrm{~m} \AA$ in the UVES spectrum of HD 84937 as reliable, such as the lines at $4762,4765,4766 \AA$, and the red triplet at 6013-6021 $\AA$. This, combined with the choice of the atmospheric models, stellar parameters, and methods to determine the abundances, may account for the somewhat different results in our study and in Sneden et al. (2016).

\section{Conclusions}

We present the first 3D NLTE analysis of Mn line formation in inhomogeneous model atmospheres. We employed three different statistical equilibrium codes, two of them to compute radiative transfer in 1D geometry (MULTI2.3 and DETAIL), whereas MULTI3D is used for detailed 3D NLTE calculations. 
The NLTE model atom was assembled using the new atomic data for different processes. We used the R-matrix method to compute new photo-ionisation cross-sections for 84 terms of $\mathrm{MnI}$, and employed them in place of hydrogenic crosssections. We also computed new collision rates for 71 terms of $\mathrm{Mn} \mathrm{I}$ interacting with $\mathrm{H}$ and for the first excited state of Mn II interacting with $\mathrm{H}$. The latter are supplemented with the data for $19 \mathrm{MnI}$ levels and the ground state of Mn II presented by Belyaev \& Voronov (2017c). We also implement the collision rates computed using the scattering-length approximation according to Kaulakys (1985); Barklem (2016). The new photo-ionisation cross-sections and the new rates of inelastic collisions represent the main difference with respect to our earlier results.

We confirm that the NLTE effects in Mn I are driven by over-ionisation. The qualitative behaviour of the departure coefficients and the NLTE abundance corrections is very similar. All Mn I lines in the optical suffer from strong NLTE effects and display positive NLTE abundance corrections, which increase with decreasing metallicity of the model atmosphere. LTE modelling underestimates Mn abundances, by approximately -0.5 dex in the models of metal-poor red giants, and to a lesser degree in the models of dwarfs. The NLTE abundance corrections are sensitive to the implementation of collision rates that affects the results at the level of $\sim 0.1$ dex. Departing from Bergemann \& Gehren (2008), we find that the new model does not produce large NLTE effects for the resonance lines of Mn I. We attribute this to the use of a tailored, ad-hoc scaling factor on collisions in the previous study that produced qualitatively similar results to our 3D NLTE calculations.

Our 3D modelling of solar Mn lines reveals unique features of line formation in the convective models, known from the previous studies (e.g. Dravins et al. 1981; Dravins 1987; Dravins \& Nordlund 1990a,b; Nordlund \& Dravins 1990). The spatially-resolved Mn lines show pronounced asymmetries. For the weaker lines, we find a strong anti-correlation between the line core depth and the line shift. The strong lines show a very broad distribution of line shifts, associated with granular motions. The bluer components are rather symmetric, but red components of the spatially-resolved features are very asymmetric and broad, tracing the differences in the line formation in the granules and in the inter-granular lanes.

We perform 1D LTE, 3D LTE, 1D NLTE, and 3D NLTE calculations for a large set of Mn lines, including the Mn II UV lines, but also the commonly-used optical lines, and the IR Mn I lines in the $H$-band. We find large differences between the four scenarios, which can be broadly summarised as follows:

- All lines of Mn I are significantly weaker in 3D NLTE, compared to 1D LTE. As a consequence, 1D LTE underestimates the $\mathrm{Mn}$ abundances by approximately -0.6 dex for the blue resonance Mn I lines, but -0.4 dex for the optical lines in the RGB models with $[\mathrm{Fe} / \mathrm{H}]=-2$. The effect is smaller for the models of dwarfs: the systematic bias incurred by 1D LTE is approximately -0.2 dex and does not change substantially with $[\mathrm{Fe} / \mathrm{H}]$.

- The commonly used blue lines of Mn II at 3488 and $3497 \AA$ are typically too weak in 1D LTE and 1D NLTE calculations, compared to 3D NLTE modelling. This effect is caused by significant line scattering, and is greatly amplified in the 3D inhomogeneous model atmospheres. Hence, 1D LTE and 1D NLTE typically overestimate Mn abundance derived from the Mn II 3488 and $3497 \AA$ lines. 3D NLTE effects depend strongly on the atmospheric parameters of a star, and, particularly in metal-poor dwarf models with $[\mathrm{Fe} / \mathrm{H}]=-2.0$, the 3D NLTE results are close to 1D LTE.

- The impact of convection is modest for the high-excitation $\mathrm{Mn}$ I lines with lower excitation potential $>2 \mathrm{eV}$. In particular, the least affected lines are those belonging to multiplets $9,23,24$, and 32 . We recommend using these lines for the abundance analysis in 1D NLTE.

- 3D LTE modelling substantially overestimates the line strength of resonance Mn I lines, compared to 1D LTE and 3D NLTE. As a consequence, the excitation imbalance reported for $\mathrm{Mn}$ I lines across a broad metallicity range (Bonifacio et al. 2009) will not be cured, but rather amplified by using 3D LTE.

- All IR $H$-band lines of Mn I suffer from strong systematic bias. 1D LTE underestimates the abundance derived from these lines by $0.2-0.35$ dex. On the other hand, the difference between 1D NLTE and 3D NLTE is only -0.15 dex, and this value does not depend on $[\mathrm{Fe} / \mathrm{H}], T_{\text {eff }}$, or $\log g$ of the model atmosphere. We suggest employing this correction in 1D NLTE studies, in order to account for 3D effects.

We derive a new 3D NLTE solar abundance of Mn, 5.52 \pm $0.03 \mathrm{dex}$, which is consistent with the CI meteoritic abundance, $5.50 \pm 0.03$ (Lodders 2003). The 1D LTE and 1D NLTE abundances are lower, $5.34 \pm 0.04 \mathrm{dex}$ in LTE, and 5.41 \pm 0.05 dex in NLTE. Our 3D NLTE value is slightly higher compared to the estimate of $5.42 \pm 0.04$ dex reported by Scott et al. (2015), however, they did not compute full 3D NLTE, but rather applied the NLTE corrections computed using a 1D model. In particular, in the latter study, we employed an Mn atom with Drawin's collision rates and hydrogenic photo-ionisation, in contrast to the detailed quantum-mechanical data in this work.

Our results for the metal-poor benchmark stars offer a considerably different picture on Mn abundances at low metallicity, contrasting with earlier 1D LTE studies. We find that 3D NLTE abundances in HD 84937, HD 140283, and HD 122563 are significantly higher. For HD 122563, we obtain a perfect ionisation balance in 3D NLTE that cannot be otherwise explained by the atomic data uncertainties and stellar parameters. The 3D NLTE Mn abundances derived from the Mn I lines are $~ 0.5-0.7$ (HD 84937, HD 140283) to 1 dex (HD 122563) higher compared to 1D LTE results. Also the 3D NLTE abundance derived from the Mn II lines are $\sim 0.15$ (HD 84937, HD 140283) to 0.4 dex (HD 122563) larger compared to 1D LTE. Effects of this magnitude are also expected for other stars in this metallicity regime. We strongly recommend applying NLTE, and, if possible, 3D NLTE radiative transfer to the analysis of Mn lines. Alternatively, the Mn I lines of certain multiplets (see above) can be used as a relatively reliable (with a bias of $\sim 0.1$ dex) diagnostics with hydrostatic models.

In the next paper in the series (Eitner et al., in prep.), we will apply the models developed in this work to a large sample of stars to explore the chemical evolution and nucleosynthesis of $\mathrm{Mn}$ in the Galaxy.

Acknowledgements. All calculations are run on MPCDF clusters Draco and Cobra. We thank Anish Amarsi for kindly providing us with the $\mathrm{H}$ collision rates computed using the Kaulakys recipe. This study is supported by SFB 881 of the DFG (subprojects A05, A10) and by the Research Council of Norway through its Centres of Excellence scheme, project number 262622. S.A.Y. and A.K.B. gratefully acknowledge support from the Ministry for Education and Science (Russian Federation), projects No. 3.5042.2017/6.7, 3.1738.2017/4.6. J.L. has received support through a grant from the Knut och och Alice Wallenberg foundation (2016.0019). Funding for the Stellar Astrophysics Centre is provided by The Danish National Research Foundation (Grant agreement no.: DNRF106). B.P. is partially supported by the CNES, Centre National d'Etudes Spatiales. 


\section{References}

Afşar, M., Sneden, C., Frebel, A., et al. 2016, ApJ, 819, 103

Amarsi, A. M., \& Asplund, M. 2017, MNRAS, 464, 264

Amarsi, A. M., Lind, K., Asplund, M., Barklem, P. S., \& Collet, R. 2016, MNRAS, 463, 1518

Amarsi, A. M., Barklem, P. S., Asplund, M., Collet, R., \& Zatsarinny, O. 2018a, A\&A, 616, A89

Amarsi, A. M., Nordlander, T., Barklem, P. S., et al. 2018b, A\&A, 615, A139

Amarsi, A. M., Barklem, P. S., Asplund, M., Collet, R., \& Zatsarinny, O. 2018c, A\&A, 616, A89

Amarsi, A. M., Nissen, P. E., Asplund, M., Lind, K., \& Barklem, P. S. 2019, A\&A, 622, L4

Anstee, S. D., \& O’Mara, B. J. 1991, MNRAS, 253, 549

Anstee, S. D., \& O'Mara, B. J. 1995, MNRAS, 276, 859

Asplund, M., Grevesse, N., Sauval, A. J., \& Scott, P. 2009, ARA\&A, 47, 481

Badnell, N. R. 1997, J. Phys. B At. Mol. Phys., 30, 1

Bagnulo, S., Jehin, E., Ledoux, C., et al. 2003, The Messenger, 114, 10

Barklem, P. S. 2007, A\&A, 462, 781

Barklem, P. S. 2016, A\&ARv, 24, 9

Barklem, P. S. 2017, Astrophysics Source Code Library [record ascl:1701.005]

Barklem, P. S., Piskunov, N., \& O’Mara, B. J. 2000, A\&AS, 142, 467

Bautista, M. A., \& Pradhan, A. K. 1995, J. Phys. B At. Mol. Phys., 28, L173

Belyaev, A. K., \& Yakovleva, S. A. 2017a, A\&A, 608, A33

Belyaev, A. K., \& Yakovleva, S. A. 2017b, A\&A, 606, A147

Belyaevc, A. K., \& Voronov, Y. V. 2017c, A\&A, 606, A106

Belyaev, A. K., Yakovleva, S. A., \& Barklem, P. S. 2014, A\&A, 572, A103

Belyaev, A. K., Voronov, Y. V., Yakovleva, S. A., et al. 2017, ApJ, 851, 59

Bergemann, M., \& Gehren, T. 2007, A\&A, 473, 291

Bergemann, M., \& Gehren, T. 2008, A\&A, 492, 823

Bergemann, M., Lind, K., Collet, R., Magic, Z., \& Asplund, M. 2012, MNRAS, 427, 27

Bergemann, M., Kudritzki, R.-P., Gazak, Z., Davies, B., \& Plez, B. 2015, ApJ, 804,113

Bergemann, M., Collet, R., Amarsi, A. M., et al. 2017, ApJ, 847, 15

Berrington, K. A., Burke, P. G., Butler, K., et al. 1987, J. Phys. B At. Mol. Phys., 20,6379

Bjørgen, J. P., \& Leenaarts, J. 2017, A\&A, 599, A118

Blackwell-Whitehead, R., \& Bergemann, M. 2007, A\&A, 472, L43

Bonifacio, P., Spite, M., Cayrel, R., et al. 2009, A\&A, 501, 519

Brueckner, K. A. 1971, ApJ, 169, 621

Butler, K., \& Giddings, J. 1985, Newsletter on Analysis of Astronomical Spectra (London: University of London), 9

Caffau, E., Ludwig, H.-G., Steffen, M., et al. 2008, A\&A, 488, 1031

Caffau, E., Maiorca, E., Bonifacio, P., et al. 2009, A\&A, 498, 877

Caffau, E., Ludwig, H.-G., Bonifacio, P., et al. 2010, A\&A, 514, A92

Caffau, E., Ludwig, H.-G., Steffen, M., Freytag, B., \& Bonifacio, P. 2011, Sol. Phys., 268, 255

Carlson, B. 1963, in Statistical Physics, eds. B. Alder, S. Fernbach, \& M. Rotenberg (Berlin: Springer), 1

Carlsson, M. 1992, ASP Conf. Ser., 26, 499

Collet, R., Magic, Z., \& Asplund, M. 2011, J. Phys. Conf. Ser., 328, 012003

Creevey, O. L., Thévenin, F., Boyajian, T. S., et al. 2012, A\&A, 545, A17

Creevey, O., Grundahl, F., Thévenin, F., et al. 2019, A\&A, 625, A33

Danilovic, S., \& Vince, I. 2005, Mem. Soc. Astron. It., 76, 949

Danilovic, S., Solanki, S. K., Livingston, W., Krivova, N., \& Vince, I. 2016, A\&A, 587, A33

Den Hartog, E. A., Lawler, J. E., Sobeck, J. S., Sneden, C., \& Cowan, J. J. 2011, ApJS, 194, 35

Dravins, D. 1987, A\&A, 172, 211

Dravins, D. 1990, A\&A, 228, 218

Dravins, D., \& Nordlund, A. 1990a, A\&A, 228, 184
Dravins, D., \& Nordlund, A. 1990b, A\&A, 228, 203

Dravins, D., Lindegren, L., \& Nordlund, A. 1981, A\&A, 96, 345

Drawin, H.-W. 1968, Z. Phys., 211, 404

Drawin, H. W. 1969, Z. Phys., 225, 470

Grupp, F. 2004a, A\&A, 420, 289

Grupp, F. 2004b, A\&A, 426, 309

Gudiksen, B. V., Carlsson, M., Hansteen, V. H., et al. 2011, A\&A, 531, A154

Gustafsson, B., Edvardsson, B., Eriksson, K., et al. 2008, A\&A, 486, 951

Holt, R. A., Scholl, T. J., \& Rosner, S. D. 1999, MNRAS, 306, 107

Johnson, J. A. 2002, ApJS, 139, 219

Karovicova, I., White, T. R., Nordlander, T., et al. 2018, MNRAS, 475, L81

Kaulakys, B. 1985, J. Phys. B At. Mol. Phys., 18, L167

Kaulakys, B. 1991, J. Phys. B At. Mol. Phys., 24, L127

Kunasz, P., \& Auer, L. H. 1988, J. Quant. Spectr. Rad. Transf., 39, 67

Kurucz, R. L., Furenlid, I., Brault, J., \& Testerman, L. 1984, Solar Flux Atlas from 296 to $1300 \mathrm{~nm}$ (New Mexico: National Solar Observatory)

Leenaarts, J., \& Carlsson, M. 2009, in The Second Hinode Science Meeting: Beyond Discovery-Toward Understanding, eds. B. Lites, M. Cheung, T. Magara, J. Mariska, \& K. Reeves, ASP Conf. Ser., 415, 87

Leenaarts, J., Carlsson, M., \& Rouppe van der Voort, L. 2012, ApJ, 749, 136

Lind, K., Bergemann, M., \& Asplund, M. 2012, MNRAS, 427, 50

Lind, K., Amarsi, A. M., Asplund, M., et al. 2017, MNRAS, 468, 4311

Livingston, W., Wallace, L., White, O. R., \& Giampapa, M. S. 2007, ApJ, 657, 1137

Lodders, K. 2003, ApJ, 591, 1220

Magic, Z., Collet, R., Asplund, M., et al. 2013, A\&A, 557, A26

Mishenina, T., Gorbaneva, T., Pignatari, M., Thielemann, F.-K., \& Korotin, S. A. 2015, MNRAS, 454, 1585

Nordlander, T., \& Lind, K. 2017, A\&A, 607, A75

Nordlund, A., \& Dravins, D. 1990, A\&A, 228, 155

Nordlund, A., \& Galsgaard, K. 1995, A 3D MHD code for parallel computers,

Tech. rep., Niels Bohr Institute, University of Copenhagen

Nordlund, A., Stein, R. F., \& Asplund, M. 2009, Liv. Rev. Sol. Phys., 6, 2

O'Mara, B. J. 1976, MNRAS, 177, 551

Osorio, Y., Barklem, P. S., Lind, K., et al. 2015, A\&A, 579, A53

Plez, B. 2012, Turbospectrum: Code for spectral synthesis, Astrophysics Source Code Library [record ascl:1205.004]

Reetz, J. K. 1999, Sauerstoff in kühlen Sternen und die chemische Entwicklung der Galaxis (Munich, Germany: LMU München)

Reilman, R. F., \& Manson, S. T. 1979, ApJS, 40, 815

Reiners, A., Mrotzek, N., Lemke, U., Hinrichs, J., \& Reinsch, K. 2016, A\&A, 587, A65

Rybicki, G. B., \& Hummer, D. G. 1991, A\&A, 245, 171

Rybicki, G. B., \& Hummer, D. G. 1992, A\&A, 262, 209

Scott, P., Asplund, M., Grevesse, N., Bergemann, M., \& Sauval, A. J. 2015, A\&A, 573, A26

Seitenzahl, I. R., Cescutti, G., Röpke, F. K., Ruiter, A. J., \& Pakmor, R. 2013 A\&A, 559, L5

Shetrone, M., Bizyaev, D., Lawler, J. E., et al. 2015, ApJS, 221, 24

Sneden, C., Cowan, J. J., Kobayashi, C., et al. 2016, ApJ, 817, 53

Steenbock, W., \& Holweger, H. 1984, A\&A, 130, 319

Stein, R. F., \& Nordlund, A. 1989, ApJ, 342, L95

Strassmeier, K. G., Ilyin, I., \& Steffen, M. 2018, A\&A, 612, A44

Unsöld, A. 1927, Z. Astrophys., 43, 563

Unsöld, A. 1955, Physik der Sternatmospharen: MIT Besonderer Berucksichtigung der Sonne (Berlin: Springer)

Verner, D. A., \& Yakovlev, D. G. 1995, A\&AS, 109, 125

Vitas, N., Viticchiè, B., Rutten, R. J., \& Vögler, A. 2009, A\&A, 499, 301

Wise, A. W., Dodson-Robinson, S. E., Bevenour, K., \& Provini, A. 2018, AJ, 156,180

Yakovleva, S. A., Barklem, P. S., \& Belyaev, A. K. 2017, MNRAS, 473, 3810 
Appendix A: Additional figures
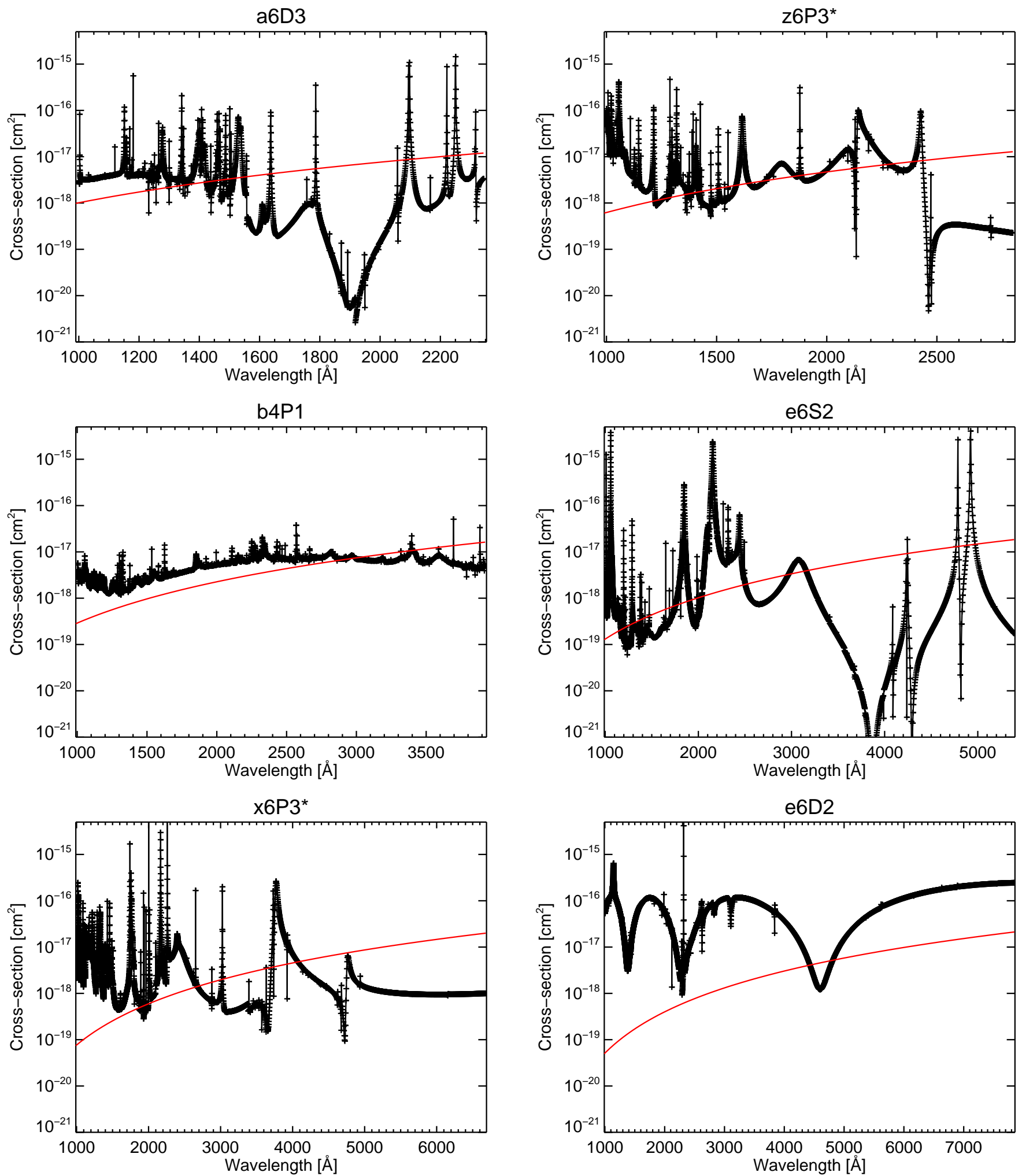

Fig. A.1. Photo-ionisation cross-sections for selected Mn I levels with quantum-mechanical data. Top to bottom: a6D3, z6P3*, b4P1, e6S2, x6P3*, and e6D2. Red lines illustrate the hydrogenic cross-sections computed using the effective principal quantum number. 

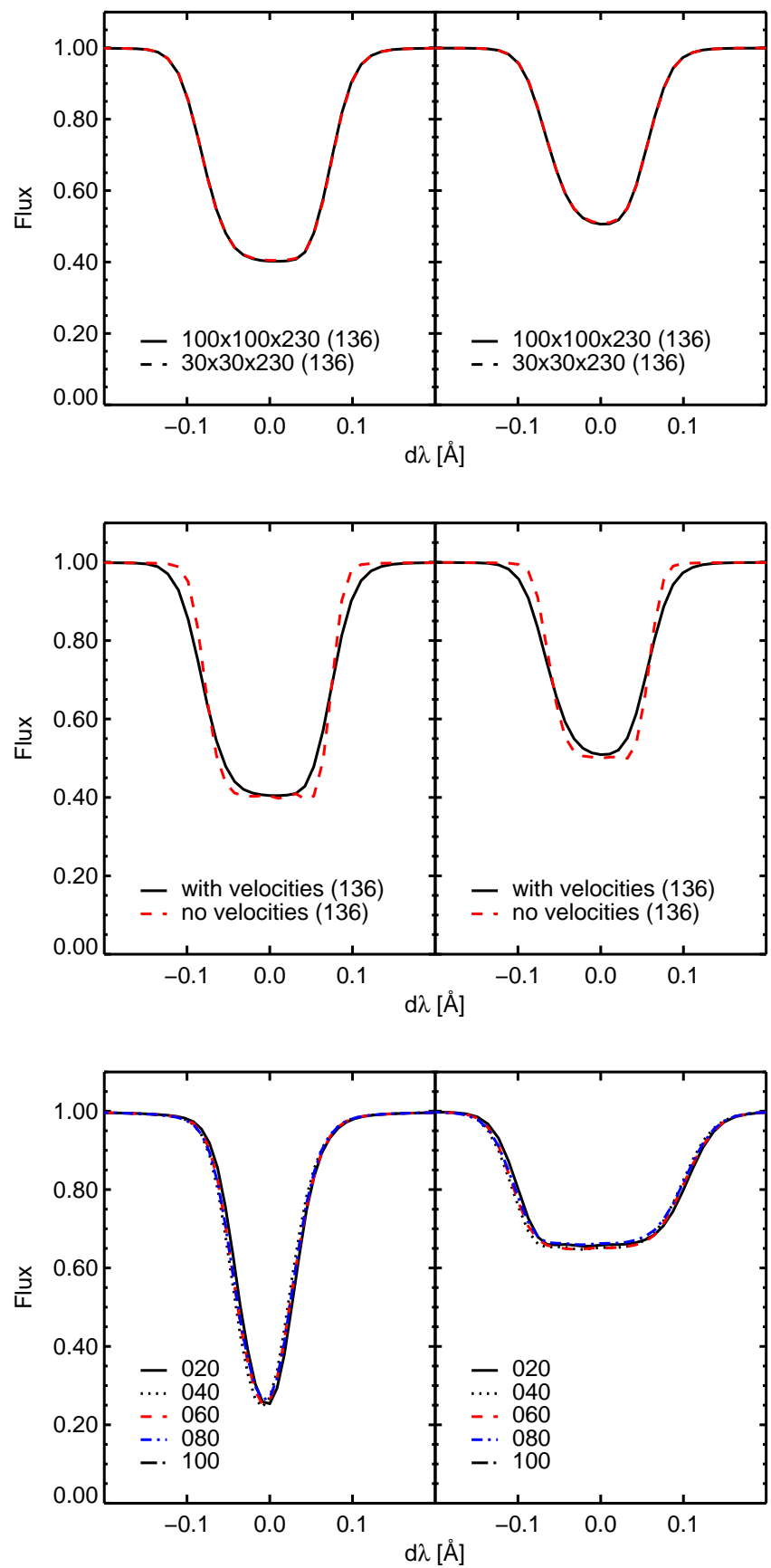

Fig. A.2. Comparison of theoretical profiles for Mn I lines at 5394 and $5432 \AA$ in 3D Sun model. Shown are the impact of the resolution of the 3D atmosphere cube (top panel) and the radiative transfer calculations with or without velocity field in opacity (middle panel). The bottom panel demonstrates the line profiles computed for five different solar snapshots selected at regular time intervals. 
M. Bergemann et al.: Observational constraints on the origin of the elements. I.

\section{Appendix B: Additional tables}

Table B.1. LS terms of target Mn II ion included in close-coupling expansion.

\begin{tabular}{|c|c|c|c|c|c|c|c|c|c|c|c|}
\hline Configuration & Term & $\begin{array}{l}\text { Energy } \\
\text { theory }\end{array}$ & $\begin{array}{l}\text { (Ryd) } \\
\text { exp. }\end{array}$ & Configuration & Term & $\begin{array}{l}\text { Energy } \\
\text { theory }\end{array}$ & $\begin{array}{l}\text { (Ryd) } \\
\text { exp. }\end{array}$ & tion & Term & $\begin{array}{l}\text { Energy } \\
\text { theory }\end{array}$ & $\begin{array}{l}\text { (Ryd) } \\
\text { exp. }\end{array}$ \\
\hline $3 d^{5}\left({ }^{6} S\right) 4 s$ & ${ }^{7} \mathrm{~S}$ & 0.0000 & 0.0000 & $3 d^{5}\left({ }^{2} G\right) 4 s$ & ${ }^{3} \mathrm{G}$ & 0.5430 & 0.4508 & $3 d^{5}\left({ }^{4} D\right) 4 p$ & ${ }^{3} \mathrm{D}^{o}$ & 0.7630 & 0.6688 \\
\hline $3 d^{5}\left({ }^{6} S\right) 4 s$ & ${ }^{5} \mathrm{~S}$ & 0.1048 & 0.0863 & $3 d^{5}\left({ }^{4} F\right) 4 s$ & ${ }^{3} \mathrm{~F}$ & 0.5491 & 0.4544 & $3 d^{5}\left({ }^{4} D\right) 4 p$ & ${ }^{3} \mathrm{~F}^{o}$ & 0.7678 & 0.672 \\
\hline $3 d^{6}$ & ${ }^{5} \mathrm{D}$ & 0.1560 & 0.1331 & $3 d^{5}\left({ }^{2} F\right) 4 s$ & ${ }^{1} \mathrm{~F}$ & 0.5495 & 0.4290 & $3 d^{5}\left({ }^{2} I\right) 4 p$ & ${ }^{3} \mathbf{I}^{o}$ & 7847 & 0.7137 \\
\hline $3 d^{5}\left({ }^{4} G\right) 4 s$ & ${ }^{5} \mathrm{G}$ & 0.2983 & 0.2513 & $3 \mathrm{~d}^{5}\left({ }^{2} \mathrm{H}\right) 4 \mathrm{~s}$ & ${ }^{1} \mathrm{H}$ & 0.5607 & 0.4698 & $3 d^{5}\left({ }^{4} P\right) 4 p$ & ${ }^{3} \mathrm{~S}^{o}$ & 0.7881 & 0.6735 \\
\hline $3 d^{6}$ & ${ }^{3} \mathrm{H}$ & 0.3230 & 0.2793 & $3 d^{5}\left({ }^{2} F\right) 4 s$ & ${ }^{3} \mathrm{~F}$ & 0.5742 & 0.4773 & $3 d^{5}\left({ }^{2} G\right) 4 s$ & ${ }^{1} \mathrm{G}$ & 0.7973 & 0.6620 \\
\hline $3 d^{6}$ & ${ }^{3} \mathrm{P}$ & 0.3358 & 0.2764 & $3 d^{5}\left({ }^{2} G\right) 4 s$ & ${ }^{1} \mathrm{G}$ & 0.5760 & 0.4798 & $3 d^{5}\left({ }^{4} D\right) 4 p$ & ${ }^{3} \mathrm{P}^{o}$ & 0.7976 & 0.6907 \\
\hline $3 d^{5}\left({ }^{4} P\right) 4 s$ & ${ }^{5} \mathrm{P}$ & 0.3406 & 0.2726 & $3 d^{6}$ & ${ }^{3} \mathrm{P}$ & 59 & 0.4853 & $3 d^{5}\left({ }^{2} I\right) 4 p$ & ${ }^{1} \mathrm{H}^{o}$ & 978 & $0.720 \mathrm{~s}$ \\
\hline $3 d^{6}$ & ${ }^{3} \mathrm{~F}$ & 0.3436 & 0.2882 & $3 d^{6}$ & ${ }^{3} \mathrm{~F}$ & 0.5929 & 0.4900 & $3 d^{5}\left({ }^{2} I\right) 4 p$ & ${ }^{3} \mathrm{H}^{o}$ & 0.8043 & 0.7263 \\
\hline $3 d^{6}$ & ${ }^{3} \mathrm{G}$ & 0.3488 & 0.3178 & $3 d^{5}\left({ }^{2} F\right) 4 s$ & ${ }^{1} \mathrm{~F}$ & 0.6204 & 0.5081 & $3 d^{5}\left(a^{2} D\right) 4 p$ & ${ }^{1} \mathrm{D}^{o}$ & 0.8247 & 0.7191 \\
\hline $3 d^{5}\left({ }^{6} S\right) 4 p$ & ${ }^{7} \mathrm{P}^{o}$ & 0.3564 & 0.3518 & $3 d^{5}\left({ }^{2} S\right) 4 s$ & ${ }^{3} \mathrm{~S}$ & 0.6371 & 0.5184 & $3 d^{5}\left(a^{2} D\right) 4 p$ & ${ }^{3} \mathrm{~F}^{o}$ & 0.8259 & 0.7260 \\
\hline $3 d^{5}\left({ }^{4} D\right) 4 s$ & ${ }^{5} \mathrm{~S}$ & 0.3660 & 0.2992 & $3 d^{5}\left({ }^{4} G\right) 4 p$ & ${ }^{5} \mathrm{G}^{o}$ & 0.6402 & 0.5878 & $3 d^{5}\left({ }^{2} I\right) 4 p$ & ${ }^{1} \mathrm{I}^{o}$ & 374 & 745 \\
\hline $3 d^{5}\left({ }^{4} G\right) 4 s$ & ${ }^{3} \mathrm{G}$ & 0.3828 & 0.3027 & $3 d^{6}$ & ${ }^{1} \mathrm{G}$ & 0.6486 & 0.5425 & $3 d^{5}\left(a^{2} F\right) 4 p$ & ${ }^{1} \mathrm{G}^{o}$ & 0.8449 & 0.7407 \\
\hline $3 d^{6}$ & ${ }^{3} \mathrm{D}$ & 0.4022 & 0.3448 & $3 d^{4} 4 s^{2}$ & ${ }^{5} \mathrm{D}$ & 0.6552 & 0.5043 & $3 d^{5}\left({ }^{4} F\right) 4 p$ & ${ }^{5} \mathrm{G}^{o}$ & 0.8508 & 0.7475 \\
\hline $3 d^{6}$ & ${ }^{1} \mathrm{I}$ & 0.4043 & 0.3528 & $3 d^{5}\left({ }^{4} G\right) 4 p$ & ${ }^{5} \mathrm{H}^{o}$ & 0.6555 & 0.5986 & $3 d^{5}\left(a^{2} D\right) 4 p$ & ${ }^{3} \mathrm{P}^{o}$ & 0.8524 & 0.7409 \\
\hline $3 d^{5}\left({ }^{4} P\right) 4 s$ & ${ }^{3} \mathrm{P}$ & 0.4133 & 0.3311 & $3 d^{5}\left({ }^{4} G\right) 4 p$ & ${ }^{5} \mathrm{~F}^{o}$ & 0.6680 & 0.6077 & $3 d^{5}\left({ }^{2} F\right) 4 p$ & ${ }^{3} \mathrm{G}^{o}$ & 0.8528 & $0.74 \mathrm{C}$ \\
\hline $3 d^{6}$ & ${ }^{1} \mathrm{G}$ & 0.4133 & 0.3545 & $3 d^{5}\left({ }^{4} P\right) 4 p$ & ${ }^{5} \mathrm{D}^{o}$ & 39 & 0.6102 & $3 d^{5}\left({ }^{4} F\right) 4 p$ & ${ }^{5} \mathrm{~F}^{o}$ & 0.8543 & 0.7473 \\
\hline $3 d^{5}\left({ }^{6} S\right) 4 p$ & ${ }^{5} \mathrm{P}^{o}$ & 0.4331 & 0.3959 & $3 d^{5}\left({ }^{4} P\right) 4 p$ & ${ }^{5} \mathrm{~S}^{o}$ & 0.6858 & 0.6099 & $3 d 5 .\left(a^{2} D\right) 4 p$ & ${ }^{1} \mathrm{~F}^{o}$ & 0.8549 & 0.7401 \\
\hline $3 d^{5}\left({ }^{2} I\right) 4 s$ & ${ }^{3} \mathrm{I}$ & 0.4373 & 0.3754 & $3 d^{5}\left({ }^{4} G\right) 4 p$ & ${ }^{3} \mathrm{~F}^{o}$ & 0.6887 & 0.6181 & $3 d^{5}\left({ }^{2} D\right) 4 p$ & ${ }^{3} \mathrm{D}^{o}$ & 0.8591 & 0.7447 \\
\hline $3 d^{5}\left({ }^{4} D\right) 4 s$ & ${ }^{3} \mathrm{D}$ & 0.4449 & 0.3628 & $3 d^{5}\left({ }^{4} G\right) 4 p$ & ${ }^{3} \mathrm{H}^{o}$ & 0.6925 & 0.6181 & $3 d^{5}\left({ }^{4} F\right) 4 p$ & ${ }^{5} \mathrm{D}^{o}$ & 0.8604 & 0.75 \\
\hline $3 d^{6}$ & ${ }^{1} \mathrm{~S}$ & 0.4478 & 0.3714 & $3 d^{5}\left({ }^{2} D\right) 4 s$ & ${ }^{3} \mathrm{D}$ & 0.6977 & 0.5702 & $3 d^{5}\left(a^{2} F\right) 4 p$ & ${ }^{3} \mathrm{~F}^{o}$ & 0.8650 & 0.7553 \\
\hline $3 d^{5}\left({ }^{2} I\right) 4 s$ & ${ }^{1} \mathrm{I}$ & 0.4720 & 0.4038 & $3 d^{5}\left({ }^{4} P\right) 4 p$ & ${ }^{5} \mathrm{P}^{o}$ & 0.7002 & 0.6231 & $3 d^{5}\left(a^{2} F\right) 4 p$ & ${ }^{3} \mathrm{D}^{o}$ & 0.8673 & 0.7541 \\
\hline $3 d^{5}\left({ }^{4} F\right) 4 s$ & ${ }^{5} \mathrm{~F}$ & 0.4810 & 0.3972 & $3 d^{5}\left({ }^{4} D\right) 4 p$ & ${ }^{5} \mathrm{~F}^{o}$ & 0.7185 & 0.6419 & $3 d^{5}\left(a^{2} G\right) 4 p$ & ${ }^{3} \mathrm{H}^{o}$ & 0.8674 & 0.7698 \\
\hline $3 d^{6}$ & ${ }^{1} \mathrm{D}$ & 0.4812 & 0.3930 & $3 d^{5}\left({ }^{4} P\right) 4 p$ & ${ }^{3} \mathrm{P}^{o}$ & 0.7193 & 0.6301 & $3 d^{5}\left({ }^{4} F\right) 4 p$ & ${ }^{3} \mathrm{G}^{o}$ & 0.8741 & 0.7646 \\
\hline $3 d^{5}\left({ }^{2} F\right) 4 s$ & ${ }^{3} \mathrm{~F}$ & 0.4965 & 0.4074 & $3 d^{5}\left({ }^{2} D\right) 4 s$ & ${ }^{1} \mathrm{D}$ & 0.7243 & 0.5975 & $3 d^{5}\left({ }^{2} \mathrm{H}\right) 4 \mathrm{p}$ & ${ }^{3} \mathrm{I}^{o}$ & 0.8824 & 0.7805 \\
\hline $3 d^{5}\left({ }^{2} D\right) 4 s$ & ${ }^{3} \mathrm{D}$ & 0.4990 & 0.3968 & $3 d^{5}\left({ }^{4} G\right) 4 p$ & ${ }^{3} \mathrm{G}^{o}$ & 0.7289 & 0.6427 & $3 d^{5}\left(a^{2} D\right) 4 p$ & ${ }^{1} \mathrm{P}^{o}$ & 0.8864 & 0.7679 \\
\hline $3 d^{6}$ & ${ }^{1} \mathrm{~F}$ & 0.5099 & 0.4492 & $3 d^{5}\left({ }^{4} D\right) 4 p$ & ${ }^{5} \mathrm{P}^{o}$ & 0.7335 & 0.6501 & $3 d^{5}\left({ }^{2} H\right) 4 p$ & ${ }^{3} \mathrm{G}^{o}$ & 0.8890 & 0.780 \\
\hline $3 d^{5}\left({ }^{2} D\right) 4 s$ & ${ }^{1} \mathrm{D}$ & 0.5254 & 0.4274 & $3 d^{5}\left({ }^{4} D\right) 4 p$ & ${ }^{5} \mathrm{D}^{o}$ & 0.7412 & 0.6579 & $3 d^{5}\left(a^{2} F\right) 4 p$ & ${ }^{1} \mathrm{D}^{o}$ & 0.8973 & 0.7779 \\
\hline $3 d^{5}\left({ }^{2} H\right) 4 s$ & ${ }^{3} \mathrm{H}$ & 0.5269 & 0.4408 & $3 d^{5}\left({ }^{4} P\right) 4 p$ & ${ }^{3} \mathrm{D}^{o}$ & 0.7431 & 0.6459 & $3 d^{5}\left({ }^{2} I\right) 4 p$ & ${ }^{1} \mathrm{H}^{o}$ & 0.9274 & 0.7212 \\
\hline
\end{tabular}

Table B.2. AUTOSTRUCTURE configuration expansions for Mn II.

Configuration expansion

$3 d^{6}, 3 d^{5} 4 s, 3 d^{5} 4 p, 3 d^{5} 4 d, 3 d^{5} 5 s, 3 d^{5} 5 p, 3 d^{4} 4 s^{2}$,

$3 \mathrm{~d}^{4} 4 \mathrm{~s} 4 \mathrm{p}, 3 \mathrm{~d}^{4} 4 \mathrm{~s} 4 \mathrm{~d}, 3 \mathrm{~d}^{4} 4 \mathrm{p}^{2}$

$\lambda_{1 \mathrm{~s}}=1.00000, \lambda_{2 \mathrm{~s}}=1.25438, \lambda_{2 \mathrm{p}}=1.10633, \lambda_{3 \mathrm{~s}}=1.10274$,

$\lambda_{3 \mathrm{p}}=1.06738, \lambda_{3 \mathrm{~d}}=1.06340, \lambda_{4 \mathrm{~s}}=1.02844, \lambda_{4 \mathrm{p}}=1.11707$,

$\lambda_{4 \mathrm{~d}}=1.46349, \lambda_{5 \mathrm{~s}}=1.00000, \lambda_{5 \mathrm{p}}=1.00000$ 
Table B.3. Scattering channels correlated to $\mathrm{MgH}$ molecular ${ }^{5} \Sigma^{+}$states, asymptotic energies ( $J$-average experimental values taken from NIST) with respect to the ground state and electronic bound energies with respect to the ionisation limit $\mathrm{Mn}^{+}\left(3 \mathrm{~d}^{5} 4 \mathrm{~s}^{5} \mathrm{~S}\right)+\mathrm{H}$.

\begin{tabular}{|c|c|c|c|}
\hline $\mathrm{j}$ & Scattering channels & $\begin{array}{l}\text { Asymptotic } \\
\text { energy }(\mathrm{eV})\end{array}$ & $\begin{array}{c}\text { Bound } \\
\text { energy }(\mathrm{eV})\end{array}$ \\
\hline 1 & $\operatorname{Mn}\left(3 d^{5} 4 s^{2} a^{6} S\right)+H$ & 0.000000 & -8.60854 \\
\hline 2 & $\operatorname{Mn}\left(3 d^{6} 4 s a^{6} D\right)+H$ & 2.145077 & -6.46346 \\
\hline 3 & $\operatorname{Mn}\left(3 d^{6} 4 s a^{4} D\right)+H$ & 2.914772 & -5.69377 \\
\hline 4 & $\operatorname{Mn}\left(3 d^{5} 4 s 4 p z^{6} P^{\circ}\right)+H$ & 3.073870 & -5.53467 \\
\hline 5 & $\operatorname{Mn}\left(3 d^{5} 4 s^{2} a^{4} G\right)+H$ & 3.134155 & -5.47438 \\
\hline 6 & $\operatorname{Mn}\left(3 d^{5} 4 s^{2} b^{4} D\right)+H$ & 3.768363 & -4.84018 \\
\hline 7 & $\operatorname{Mn}\left(3 d^{5} 4 s 4 p z^{4} P^{\circ}\right)+H$ & 3.849324 & -4.75921 \\
\hline 8 & $\operatorname{Mn}\left(3 d^{5} 4 s 4 p y^{6} P^{\circ}\right)+H$ & 4.430886 & -4.17765 \\
\hline 9 & $\operatorname{Mn}\left(3 d^{6} 4 s b^{4} G\right)+H$ & 4.663140 & -3.94540 \\
\hline 10 & $\operatorname{Mn}\left(3 d^{5} 4 s 5 s e^{6} S\right)+H$ & 5.133436 & -3.47510 \\
\hline 11 & $\operatorname{Mn}\left(3 d^{5} 4 s 4 d e^{6} D\right)+H$ & 5.853606 & -2.75493 \\
\hline 12 & $\operatorname{Mn}\left(3 d^{5} 4 s 5 p w^{6} P^{\circ}\right)+H$ & 5.897434 & -2.71110 \\
\hline 13 & $\operatorname{Mn}\left(3 d^{5} 4 s 4 p y^{6} F^{\circ}\right)+H$ & 5.972531 & -2.63601 \\
\hline 14 & $\operatorname{Mn}\left(3 d^{5} 4 s 5 s f^{6} S\right)+H$ & 6.126726 & -2.48181 \\
\hline 15 & $\operatorname{Mn}\left(3 d^{5} 4 s 5 s e^{4} S\right)+H$ & 6.148568 & -2.45997 \\
\hline 16 & $\operatorname{Mn}\left(3 d^{5} 4 s 4 p v^{6} P^{\circ}\right)+H$ & 6.196287 & -2.41225 \\
\hline 17 & $\operatorname{Mn}\left(3 d^{5} 4 s 4 p z^{4} H^{\circ}\right)+H$ & 6.209216 & -2.39932 \\
\hline 18 & $\operatorname{Mn}\left(3 d^{5} 4 s 4 p y^{4} F^{\circ}\right)+H$ & 6.243758 & -2.36478 \\
\hline 19 & $\operatorname{Mn}\left(3 d^{5} 4 s 4 p x^{6} F^{\circ}\right)+H$ & 6.328611 & -2.27993 \\
\hline 20 & $\operatorname{Mn}\left(3 d^{5} 4 s 6 s g^{6} S\right)+H$ & 6.311380 & -2.29716 \\
\hline 21 & $\operatorname{Mn}\left(3 d^{5} 4 s 4 p x^{4} P^{\circ}\right)+H$ & 6.371961 & -2.23658 \\
\hline 22 & $\operatorname{Mn}\left(3 d^{5} 4 s 4 p u^{6} P^{\circ}\right)+H$ & 6.466860 & -2.14168 \\
\hline 23 & $\operatorname{Mn}\left(3 d^{5} 4 s 5 d f^{6} D\right)+H$ & 6.537766 & -2.07077 \\
\hline 24 & $\operatorname{Mn}\left(3 d^{5} 4 s 4 f w^{6} F^{\circ}\right)+H$ & 6.568422 & -2.04012 \\
\hline 25 & $\operatorname{Mn}\left(3 d^{5} 4 s 6 p t^{6} P^{\circ}\right)+H$ & 6.606162 & -2.00238 \\
\hline 26 & $\operatorname{Mn}\left(3 d^{5} 4 s 7 s h^{6} S\right)+H$ & 6.752220 & -1.85632 \\
\hline 27 & $\operatorname{Mn}\left(3 d^{5} 4 s 4 d g^{6} D\right)+H$ & 6.812434 & -1.79610 \\
\hline 28 & $\operatorname{Mn}\left(3 d^{5} 4 s 5 p w^{4} P^{\circ}\right)+H$ & 6.868987 & -1.73955 \\
\hline 29 & $\operatorname{Mn}\left(3 d^{5} 4 s 5 f v^{6} F^{\circ}\right)+H$ & 6.880172 & -1.72837 \\
\hline 30 & $\operatorname{Mn}\left(3 d^{5} 4 s 7 p r^{6} P^{\circ}\right)+H$ & 6.880244 & -1.72829 \\
\hline 31 & $\operatorname{Mn}\left(3 d^{5} 4 s 6 d h^{6} D\right)+H$ & 6.904357 & -1.70418 \\
\hline 32 & $\operatorname{Mn}\left(3 d^{5} 4 s 5 p t^{4} P^{\circ}\right)+H$ & 6.934452 & -1.67409 \\
\hline 33 & $\operatorname{Mn}\left(3 d^{5} 4 s 5 p s^{6} P^{\circ}\right)+H$ & 6.944067 & -1.66447 \\
\hline 34 & $\operatorname{Mn}\left(3 d^{6} 5 s i^{6} D\right)+H$ & 6.989790 & -1.61875 \\
\hline 35 & $\operatorname{Mn}\left(3 d^{5} 4 s 6 f u^{6} F^{\circ}\right)+H$ & 7.050626 & -1.55791 \\
\hline 36 & $\operatorname{Mn}\left(3 d^{5} 4 s 8 p q^{6} P^{\circ}\right)+H$ & 7.058384 & -1.55016 \\
\hline 37 & $\operatorname{Mn}\left(3 d^{5} 4 s 4 p v^{4} P^{\circ}\right)+H$ & 7.116923 & -1.49162 \\
\hline 38 & $\operatorname{Mn}\left(3 d^{5} 4 s 7 f t^{6} F^{\circ}\right)+H$ & 7.153545 & -1.45499 \\
\hline 39 & $\operatorname{Mn}\left(3 d^{5} 4 s 9 p p^{6} P^{\circ}\right)+H$ & 7.156234 & -1.45230 \\
\hline 40 & $\operatorname{Mn}\left(3 d^{5} 4 s 10 p o^{6} P^{\circ}\right)+H$ & 7.220870 & -1.38767 \\
\hline 41 & $\operatorname{Mn}\left(3 d^{5} 4 s 11 p^{6} P^{\circ}\right)+H$ & 7.265446 & -1.34309 \\
\hline 42 & $\operatorname{Mn}\left(3 d^{5} 4 s 12 p^{6} P^{\circ}\right)+H$ & 7.297423 & -1.31112 \\
\hline 43 & $\operatorname{Mn}\left(3 d^{5} 4 s 13 p^{6} P^{\circ}\right)+H$ & 7.321116 & -1.28742 \\
\hline 44 & $\operatorname{Mn}\left(3 d^{5} 4 s 14 p^{6} P^{\circ}\right)+H$ & 7.339133 & -1.26941 \\
\hline
\end{tabular}

Table B.3. continued.

\begin{tabular}{|c|c|c|c|}
\hline $\mathrm{j}$ & Scattering channel & $\begin{array}{l}\text { Asymptotic } \\
\text { energy }(\mathrm{eV})\end{array}$ & $\begin{array}{c}\text { Bound } \\
\text { energy }(\mathrm{eV})\end{array}$ \\
\hline 45 & $\operatorname{Mn}\left(3 d^{5} 4 s 15 p^{6} P^{\circ}\right)+H$ & 7.351767 & -1.25677 \\
\hline 46 & $\operatorname{Mn}\left(3 d^{5} 4 s 16 p^{6} P^{\circ}\right)+H$ & 7.364333 & -1.24421 \\
\hline 47 & $\operatorname{Mn}\left(3 d^{5} 4 s 17 p^{6} P^{\circ}\right)+H$ & 7.373379 & -1.23516 \\
\hline 48 & $\operatorname{Mn}\left(3 d^{5} 4 s 18 p^{6} P^{\circ}\right)+H$ & 7.380681 & -1.22786 \\
\hline 49 & $\operatorname{Mn}\left(3 d^{5} 4 s 19 p^{6} P^{\circ}\right)+H$ & 7.386776 & -1.22176 \\
\hline 50 & $\operatorname{Mn}\left(3 d^{5} 4 s 20 p^{6} P^{\circ}\right)+H$ & 7.391888 & -1.21665 \\
\hline 51 & $\operatorname{Mn}\left(3 d^{5} 4 s 21 p^{6} P^{\circ}\right)+H$ & 7.396210 & -1.21233 \\
\hline 52 & $\operatorname{Mn}\left(3 d^{5} 4 s 22 p^{6} P^{\circ}\right)+H$ & 7.399904 & -1.20864 \\
\hline 53 & $\operatorname{Mn}\left(3 d^{5} 4 s 23 p^{6} P^{\circ}\right)+H$ & 7.403078 & -1.20546 \\
\hline 54 & $\operatorname{Mn}\left(3 d^{5} 4 s 24 p^{6} P^{\circ}\right)+H$ & 7.405828 & -1.20271 \\
\hline 55 & $\operatorname{Mn}\left(3 d^{5} 4 s 25 p^{6} P^{\circ}\right)+H$ & 7.408231 & -1.20031 \\
\hline 56 & $\operatorname{Mn}\left(3 d^{5} 4 s 26 p^{6} P^{\circ}\right)+H$ & 7.410336 & -1.19820 \\
\hline 57 & $\operatorname{Mn}\left(3 d^{5} 4 s 27 p^{6} P^{\circ}\right)+H$ & 7.412199 & -1.19634 \\
\hline 58 & $\operatorname{Mn}\left(3 d^{5} 4 s 28 p^{6} P^{\circ}\right)+H$ & 7.413848 & -1.19469 \\
\hline 59 & $\operatorname{Mn}\left(3 d^{5} 4 s 29 p^{6} P^{\circ}\right)+H$ & 7.415318 & -1.19322 \\
\hline 60 & $\operatorname{Mn}\left(3 d^{5} 4 s 30 p^{6} P^{\circ}\right)+H$ & 7.416628 & -1.19191 \\
\hline 61 & $\operatorname{Mn}\left(3 d^{5} 4 s 31 p^{6} P^{\circ}\right)+H$ & 7.417802 & -1.19074 \\
\hline 62 & $\operatorname{Mn}\left(3 d^{5} 4 s 32 p^{6} P^{\circ}\right)+H$ & 7.418876 & -1.18966 \\
\hline 63 & $\operatorname{Mn}\left(3 d^{5} 4 s 33 p^{6} P^{\circ}\right)+H$ & 7.419839 & -1.18870 \\
\hline 64 & $\operatorname{Mn}\left(3 d^{5} 4 s 34 p^{6} P^{\circ}\right)+H$ & 7.420701 & -1.18784 \\
\hline 65 & $\operatorname{Mn}\left(3 d^{5} 4 s 35 p^{6} P^{\circ}\right)+H$ & 7.421504 & -1.18704 \\
\hline 66 & $\operatorname{Mn}\left(3 d^{5} 4 s 36 p^{6} P^{\circ}\right)+H$ & 7.422245 & -1.18629 \\
\hline 67 & $\operatorname{Mn}\left(3 d^{5} 4 s 37 p^{6} P^{\circ}\right)+H$ & 7.422891 & -1.18565 \\
\hline 68 & $\operatorname{Mn}\left(3 d^{5} 4 s 38 p^{6} P^{\circ}\right)+H$ & 7.423498 & -1.18504 \\
\hline 69 & $\operatorname{Mn}\left(3 d^{5} 4 s 39 p^{6} P^{\circ}\right)+H$ & 7.424055 & -1.18448 \\
\hline 70 & $\operatorname{Mn}\left(3 d^{5} 4 s 40 p{ }^{6} P^{\circ}\right)+H$ & 7.424578 & -1.18396 \\
\hline 71 & $\operatorname{Mn}\left(3 d^{5} 4 s 41 p^{6} P^{\circ}\right)+H$ & 7.425049 & -1.18349 \\
\hline 72 & $\mathrm{Mn}^{+}\left(3 \mathrm{~d}^{5} 4 \mathrm{~s}^{5} \mathrm{~S}\right)+\mathrm{H}^{-}$ & 7.854539 & -0.754 \\
\hline
\end{tabular}


M. Bergemann et al.: Observational constraints on the origin of the elements. I.

Table B.4. Scattering channels correlated to $\mathrm{MgH}^{+}$molecular ${ }^{6} \Sigma^{+}$ states, asymptotic energies $(J$-average experimental values taken from NIST with respect to the ground state and electronic bound energies with respect to the ionisation limit $\left.\mathrm{Mn}^{2+}\left(3 \mathrm{~d}^{5}{ }^{6} \mathrm{~S}\right)+\mathrm{H}\right)$.

\begin{tabular}{|c|c|c|c|}
\hline $\mathrm{j}$ & Scattering channel & $\begin{array}{l}\text { Asymptotic } \\
\text { energy }(\mathrm{eV})\end{array}$ & $\begin{array}{c}\text { Bound } \\
\text { energy }(\mathrm{eV})\end{array}$ \\
\hline 1 & $\mathrm{Mn}^{+}\left(3 \mathrm{~d}^{5} 4 \mathrm{~s} \mathrm{a}^{7} \mathrm{~S}\right)+\mathrm{H}$ & 0.000000 & -15.6400 \\
\hline 2 & $\mathrm{Mn}^{+}\left(3 \mathrm{~d}^{5} 4 \mathrm{~s} \mathrm{a}^{5} \mathrm{~S}\right)+\mathrm{H}$ & 1.174501 & -14.4655 \\
\hline 3 & $\operatorname{Mn}^{+}\left(3 d^{6} a^{5} D\right)+H$ & 1.808479 & -13.8315 \\
\hline 4 & $\mathrm{Mn}^{+}\left(3 \mathrm{~d}^{5} 4 \mathrm{~s} \mathrm{a}^{5} \mathrm{G}\right)+\mathrm{H}$ & 3.418429 & -12.2216 \\
\hline 5 & $\mathrm{Mn}^{+}\left(3 \mathrm{~d}^{5} 4 \mathrm{~s} \mathrm{~b}^{5} \mathrm{D}\right)+\mathrm{H}$ & 4.070230 & -11.5698 \\
\hline 6 & $\mathrm{Mn}^{+}\left(3 \mathrm{~d}^{5} 4 p \mathrm{z}^{7} \mathrm{P}^{\circ}\right)+\mathrm{H}$ & 4.787521 & -10.8525 \\
\hline 7 & $\mathrm{Mn}^{+}\left(3 \mathrm{~d}^{5} 4 p \mathrm{z}^{5} \mathrm{P}^{\circ}\right)+\mathrm{H}$ & 5.386607 & -10.2534 \\
\hline 8 & $\mathrm{Mn}^{+}\left(3 \mathrm{~d}^{5} 4 p \mathrm{z}^{5} \mathrm{H}^{\circ}\right)+\mathrm{H}$ & 8.145176 & -7.49481 \\
\hline 9 & $\mathrm{Mn}^{+}\left(3 \mathrm{~d}^{5} 4 p \mathrm{z}^{5} \mathrm{~F}^{\circ}\right)+\mathrm{H}$ & 8.267108 & -7.37288 \\
\hline 10 & 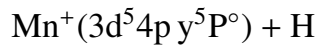 & 8.476972 & -7.16302 \\
\hline 11 & $\mathrm{Mn}^{+}\left(3 \mathrm{~d}^{5} 4 p \mathrm{y}^{5} \mathrm{~F}^{\circ}\right)+\mathrm{H}$ & 8.734600 & -6.90539 \\
\hline 12 & $\mathrm{Mn}^{+}\left(3 \mathrm{~d}^{5} 4 \mathrm{px^{5 }} \mathrm{P}^{\circ}\right)+\mathrm{H}$ & 8.845407 & -6.79458 \\
\hline 13 & $\mathrm{Mn}^{+}\left(3 \mathrm{~d}^{5} 5 \mathrm{se}^{7} \mathrm{~S}\right)+\mathrm{H}$ & 9.244271 & -6.39572 \\
\hline 14 & $\mathrm{Mn}^{+}\left(3 \mathrm{~d}^{5} 5 \mathrm{~s} \mathrm{e}^{5} \mathrm{~S}\right)+\mathrm{H}$ & 9.469252 & -6.17074 \\
\hline 15 & $\mathrm{Mn}^{+}\left(3 \mathrm{~d}^{5} 4 \mathrm{de}^{7} \mathrm{D}\right)+\mathrm{H}$ & 9.863794 & -5.77620 \\
\hline 16 & $\mathrm{Mn}^{+}\left(3 \mathrm{~d}^{5} 4 p \mathrm{x}^{5} \mathrm{~F}^{\circ}\right)+\mathrm{H}$ & 10.16684 & -5.47314 \\
\hline 17 & $\mathrm{Mn}^{+}\left(3 \mathrm{~d}^{5} 4 d \mathrm{e}^{5} \mathrm{D}\right)+\mathrm{H}$ & 10.18466 & -5.45532 \\
\hline 18 & $\mathrm{Mn}^{+}\left(3 \mathrm{~d}^{5} 5 \mathrm{px^{7 }} \mathrm{P}^{\circ}\right)+\mathrm{H}$ & 10.66097 & -4.97902 \\
\hline 19 & $M n^{+}\left(3 d^{5} 5 p v^{5} P^{\circ}\right)+H$ & 11.05872 & -4.58127 \\
\hline 20 & $\mathrm{Mn}^{+}\left(3 \mathrm{~d}^{5} 6 s \mathrm{f}^{7} S\right)+\mathrm{H}$ & 12.11680 & -3.52319 \\
\hline 21 & $\mathrm{Mn}^{+}\left(3 \mathrm{~d}^{5} 6 \mathrm{~s} \mathrm{f}^{5} \mathrm{~S}\right)+\mathrm{H}$ & 12.20133 & -3.43865 \\
\hline 22 & $\mathrm{Mn}^{+}\left(3 \mathrm{~d}^{5} 4 f \mathrm{z}^{7} \mathrm{~F}^{\circ}\right)+\mathrm{H}$ & 12.20296 & -3.43702 \\
\hline 23 & $\mathrm{Mn}^{+}\left(3 \mathrm{~d}^{5} 4 f \mathrm{e}^{5} \mathrm{~F}^{\circ}\right)+\mathrm{H}$ & 12.20797 & -3.43202 \\
\hline 24 & $\mathrm{Mn}^{+}\left(3 \mathrm{~d}^{5} 5 \mathrm{df}^{7} \mathrm{D}\right)+\mathrm{H}$ & 12.38580 & -3.25419 \\
\hline 25 & $\mathrm{Mn}^{+}\left(3 d^{5} 5 d f^{5} D\right)+H$ & 12.48374 & -3.15624 \\
\hline 26 & $\mathrm{Mn}^{+}\left(3 \mathrm{~d}^{5} 5 \mathrm{~s} \mathrm{e}^{5} \mathrm{G}\right)+\mathrm{H}$ & 12.58296 & -3.05702 \\
\hline 27 & $\mathrm{Mn}^{+}\left(3 \mathrm{~d}^{5} 4 d \mathrm{e}^{5} \mathrm{I}\right)+\mathrm{H}$ & 13.20636 & -2.43362 \\
\hline 28 & $\mathrm{Mn}^{+}\left(3 d^{5} 5 s g^{5} \mathrm{D}\right)+\mathrm{H}$ & 13.25794 & -2.38204 \\
\hline 29 & $\mathrm{Mn}^{+}\left(3 \mathrm{~d}^{5} 7 \mathrm{sg}^{7} \mathrm{~S}\right)+\mathrm{H}$ & 13.40594 & -2.23404 \\
\hline 30 & $\mathrm{Mn}^{+}\left(3 \mathrm{~d}^{5} 5 \mathrm{f} \mathrm{y}^{7} \mathrm{~F}^{\circ}\right)+\mathrm{H}$ & 13.44114 & -2.19885 \\
\hline 31 & $\mathrm{Mn}^{+}\left(3 \mathrm{~d}^{5} 5 \mathrm{ff}^{5} \mathrm{~F}^{\circ}\right)+\mathrm{H}$ & 13.44490 & -2.19509 \\
\hline 32 & $\mathrm{Mn}^{+}\left(3 \mathrm{~d}^{5} 7 \mathrm{sg}^{5} \mathrm{~S}\right)+\mathrm{H}$ & 13.44576 & -2.19423 \\
\hline 33 & $\mathrm{Mn}^{+}\left(3 \mathrm{~d}^{5} 5 \mathrm{~g} \mathrm{e}^{7} \mathrm{G}\right)+\mathrm{H}$ & 13.45920 & -2.18079 \\
\hline 34 & $\mathrm{Mn}^{+}\left(3 \mathrm{~d}^{5} 5 \mathrm{g \textrm {f } ^ { 5 }} \mathrm{G}\right)+\mathrm{H}$ & 13.45925 & -2.18074 \\
\hline 35 & $\mathrm{Mn}^{+}\left(3 \mathrm{~d}^{5} 6 \mathrm{~d} \mathrm{~g}^{7} \mathrm{D}\right)+\mathrm{H}$ & 13.54475 & -2.09524 \\
\hline 36 & $\mathrm{Mn}^{+}\left(3 \mathrm{~d}^{5} 5 \mathrm{ps}^{5} \mathrm{~F}^{\circ}\right)+\mathrm{H}$ & 14.09071 & -1.54927 \\
\hline 37 & $\mathrm{Mn}^{+}\left(3 \mathrm{~d}^{5} 8 s \mathrm{~h}^{7} \mathrm{~S}\right)+\mathrm{H}$ & 14.09672 & -1.54327 \\
\hline 38 & $\mathrm{Mn}^{+}\left(3 \mathrm{~d}^{5} 6 f \mathrm{x}^{7} \mathrm{~F}^{\circ}\right)+\mathrm{H}$ & 14.11439 & -1.52559 \\
\hline 39 & $\mathrm{Mn}^{+}\left(3 d^{5} 6 g \mathrm{f}^{7} \mathrm{G}\right)+\mathrm{H}$ & 14.12574 & -1.51425 \\
\hline 40 & $\mathrm{Mn}^{+}\left(3 \mathrm{~d}^{5} 6 \mathrm{~g} \mathrm{~g}^{5} \mathrm{G}\right)+\mathrm{H}$ & 14.12579 & -1.51420 \\
\hline 41 & $\mathrm{Mn}^{+}\left(3 \mathrm{~d}^{5} 6 f \mathrm{~g}^{5} \mathrm{~F}^{\circ}\right)+\mathrm{H}$ & 14.13750 & -1.50249 \\
\hline 42 & $\mathrm{Mn}^{+}\left(3 d^{5} 7 d h^{5} \mathrm{D}\right)+\mathrm{H}$ & 14.25117 & -1.38882 \\
\hline 43 & $\mathrm{Mn}^{2+}\left(3 \mathrm{~d}^{5}{ }^{6} \mathrm{~S}\right)+\mathrm{H}^{-}$ & 14.88599 & -0.754 \\
\hline
\end{tabular}

Table B.5. NLTE abundance corrections for MARCS models with $T_{\text {eff }}=4500 \mathrm{~K}$ and $\log g=1.5$ dex.

\begin{tabular}{ccccc}
\hline \hline$\lambda(\AA)$ & {$[\mathrm{Fe} / \mathrm{H}]$} & $\mathrm{CH}$ excl. & CH0 excl. & CH/CH0 incl. \\
\hline 4030 & 0 & 0.157 & 0.176 & 0.153 \\
4033 & -1 & 0.212 & 0.236 & 0.222 \\
4034 & -2 & 0.378 & 0.434 & 0.426 \\
& -3 & 0.473 & 0.483 & 0.477 \\
& 0 & 0.198 & 0.173 & 0.156 \\
4783 & -1 & 0.189 & 0.214 & 0.203 \\
4823 & -2 & 0.333 & 0.366 & 0.358 \\
& -3 & 0.452 & 0.462 & 0.456 \\
6013 & 0 & 0.203 & 0.184 & 0.174 \\
6016 & -1 & 0.304 & 0.288 & 0.277 \\
6021 & -2 & 0.372 & 0.370 & 0.364 \\
& -3 & 0.382 & 0.396 & 0.392 \\
& 0 & -0.013 & -0.014 & -0.013 \\
3488 & -1 & -0.035 & -0.035 & -0.035 \\
& -2 & -0.078 & -0.079 & -0.079 \\
& -3 & 0.076 & 0.074 & 0.074 \\
\hline
\end{tabular}

Notes. See Fig. 9 in the main text.

Table B.6. NLTE abundance corrections for MARCS models with $T_{\text {eff }}=6000 \mathrm{~K}$ and $\log g=4.0$ dex.

\begin{tabular}{ccccc}
\hline \hline$\lambda(\AA)$ & {$[\mathrm{Fe} / \mathrm{H}]$} & $\mathrm{CH}$ excl. & CH0 excl. & CH/CH0 incl. \\
\hline 4030 & 0 & 0.043 & 0.078 & 0.042 \\
4033 & -1 & -0.043 & 0.002 & -0.033 \\
4034 & -2 & 0.124 & 0.183 & 0.113 \\
& -3 & 0.469 & 0.529 & 0.481 \\
& 0 & 0.002 & 0.038 & 0.014 \\
4783 & -1 & 0.135 & 0.186 & 0.154 \\
4823 & -2 & 0.272 & 0.308 & 0.274 \\
& -3 & 0.315 & 0.354 & 0.317 \\
6013 & 0 & 0.123 & 0.109 & 0.090 \\
6016 & -1 & 0.220 & 0.226 & 0.205 \\
6021 & -2 & 0.280 & 0.341 & 0.322 \\
& -3 & 0.288 & 0.395 & 0.378 \\
& 0 & -0.017 & -0.017 & -0.016 \\
3488 & -1 & -0.082 & -0.080 & -0.079 \\
& -2 & -0.038 & -0.041 & -0.040 \\
& -3 & 0.098 & 0.093 & 0.093 \\
\hline
\end{tabular}

Notes. See Fig. 9 in the main text. 
A\&A 631, A80 (2019)

Table B.7. NLTE abundance corrections for MARCS models with $T_{\text {eff }}=4500 \mathrm{~K}$ and $\log g=1.5 \mathrm{dex}$.

\begin{tabular}{cccc}
\hline \hline$\lambda(\AA)$ & {$[\mathrm{Fe} / \mathrm{H}]$} & Reference atom & No Kaulakys \\
\hline 4030 & 0 & 0.138 & 0.153 \\
4033 & -1 & 0.223 & 0.222 \\
4034 & -2 & 0.401 & 0.426 \\
& -3 & 0.396 & 0.477 \\
& 0 & 0.132 & 0.156 \\
4783 & -1 & 0.225 & 0.203 \\
4823 & -2 & 0.383 & 0.358 \\
& -3 & 0.438 & 0.456 \\
6013 & 0 & 0.168 & 0.174 \\
6016 & -1 & 0.292 & 0.277 \\
6021 & -2 & 0.416 & 0.364 \\
& -3 & 0.442 & 0.392 \\
3488 & 0 & -0.012 & -0.013 \\
& -1 & -0.035 & -0.035 \\
& -2 & -0.078 & -0.079 \\
\hline
\end{tabular}

Notes. See Fig. 10 in the main text.
Table B.8. NLTE abundance corrections for MARCS models with $T_{\text {eff }}=6000 \mathrm{~K}$ and $\log g=4.0$ dex.

\begin{tabular}{cccc}
\hline \hline$\lambda(\AA)$ & {$[\mathrm{Fe} / \mathrm{H}]$} & Reference atom & No Kaulakys \\
\hline 4030 & 0 & 0.029 & 0.042 \\
4033 & -1 & -0.015 & -0.033 \\
4034 & -2 & 0.078 & 0.113 \\
& -3 & 0.423 & 0.481 \\
& 0 & 0.013 & 0.014 \\
4783 & -1 & 0.118 & 0.154 \\
4823 & -2 & 0.236 & 0.274 \\
& -3 & 0.292 & 0.317 \\
6013 & 0 & 0.048 & 0.090 \\
6016 & -1 & 0.134 & 0.205 \\
6021 & -2 & 0.269 & 0.322 \\
& -3 & 0.344 & 0.378 \\
3488 & 0 & -0.016 & -0.016 \\
& -1 & -0.079 & -0.079 \\
& -2 & -0.040 & -0.040 \\
& -3 & 0.093 & 0.093 \\
\hline
\end{tabular}

Notes. See Fig. 10 in the main text. 